\title{
MEDIATING EFFECTS OF THE RELATIONSHIP BETWEEN SCHOOL-BASED PROFESSIONALS' ROLE, KNOWLEDGE, AND SELF-EFFICACY AS RELATED TO NON-SUICIDAL SELF-INJURY
}

A Dissertation
presented to
the Faculty of the Graduate School
at the University of Missouri-Columbia
In Partial Fulfillment
of the Requirements for the Degree
Doctor of Philosophy
CAREN R. MOORE
Dr. Craig Frisby, Dissertation Supervisor

MAY 2015 
(C) Copyright by Caren R. Moore 2015

All Rights Reserved 
The undersigned, appointed by the dean of the Graduate School, have examined the dissertation entitled

\section{MEDIATING EFFECTS OF THE RELATIONSHIP BETWEEN SCHOOL-BASED PROFESSIONALS' ROLE, KNOWLEDGE, AND SELF-EFFICACY \\ AS RELATED TO NON-SUICIDAL SELF-INJURY}

presented by Caren R. Moore, a candidate for the degree of doctor of philosophy of Educational and Counseling Psychology, and hereby certify that, in their opinion, it is worthy of acceptance.

Professor Craig Frisby, Chair

Professor Charles Borduin

Professor Steven Osterlind

Professor Wendy Reinke

Professor Stephen Whitney

Professor Barbara Williamson 


\section{DEDICATION}

This dissertation and the resulting doctorate degree are dedicated to the one person who sacrificed everything in order for me to make a better life for us, my daughter, Tiana. Tiana you have had to share me with various universities your entire life and now we have made it to the end. I could not have gotten this far without your love and encouragement. Thank you sweetheart and Mommy loves you very much! To my other daughter, Lauryn, I dedicate this dissertation as an example of faith, hard work, and desire. Dream big and I do mean real big. You can do anything you set your mind to, honey! I did. Mommy loves you!

This dissertation is also dedicated to my wonderful family that cried with me and supported me to the end. A sincere thank you goes to my wonderful Mother, Katherine, Grandmothers, Catherine and Nellie, Aunt Doris, Cousins Tammi, Kelley, and Dwight, my Brother, Charles Jr. and my two dear Fathers, Charles Sr. and Wayne. Thank you all for believing in me.

Last but certainly not least this is dedicated to my wonderful friends that stuck by me through the tough times and encouraged me to excel. To Todd Robinson, Vanessa Nozinor, Keacha Payne-Freeman, Cyndi Frisby, and Adrienne Riddle, I thank you.

I love you all. 


\section{ACKNOWLEDGEMENTS}

I would like to thank Professor Craig Frisby for your unfailing support over the years as I pursued my doctoral studies. From the weekend advising sessions to helpful phone calls, you have always been one of my staunch supporters from day one. Thank you for believing in me.

I would also like to thank Professors Steve Whitney, Barbara Williamson, and Wendy Reinke, for constructive and helpful feedback. Your support during this process speaks volumes to the dedication and pride you all take in ensuring students succeed.

I would like to acknowledge Mrs. Natalie Fornelli and Mr. Mark Stringer for your support, faith, and encouragement during this process. The Missouri Department of Mental Health has supported me in so many ways and I would be remiss if you were not properly recognized. Moreover, Natalie, thank you for being you!

A special acknowledgement goes to Professor Phil Wood in the Psychology Department for his statistical consulting time. I thank you.

Finally, Professor Charles Borduin, you have been a great supporter and afforded me a number of once in a lifetime clinical and academic opportunities. Thank you for seeing things in me that I initially did not see in myself. Your confidence in my abilities and support mean the world to me. 


\section{TABLE OF CONTENTS}

$\begin{array}{ll}\text { Acknowledgements } & \text { ii }\end{array}$

List of Tables viii

List of Figures $\quad \mathrm{x}$

$\begin{array}{ll}\text { Abstract } & \text { xiii }\end{array}$

Chapter Page

1. Introduction 1

Spotlight on Non-suicidal Self-Injurious Behaviors

Non-Suicidal Self-Injurious Behaviors and Mental Health Training of SchoolBased Professionals

Applications of Non-Suicidal Self-Injury Knowledge for School-Based Professionals

2. Literature Review

Defining Non-Suicidal Self-Injury

Distinguishing Features of Non-Suicidal Self-Injury

Non- Suicidal Self-Injury Prevalence

Characteristics of Individuals Who Engage in Non-Suicidal Self-Injurious Behaviors

Psychiatric disorders

Age

Gender

Race and Ethnicity

Socioeconomic Status

Similarities and Differences between Suicide and Non-Suicidal Self-Injury Assessment of Non-Suicidal Self-Injurious Behaviors 
Treatment Options for Individuals Who Engage in Non-Suicidal Self-Injurious Behaviors

Behavioral Therapy

Psychopharmacological Options

School-Based Interventions

Variables Associated with Non-Suicidal Self-Injurious Behaviors

Non-Suicidal Self-Injury Through an Ecological Orientation Lens

School-Based Health and Mental Health Professionals Knowledge of Self-Injury

School-Based Professionals Serving Students: Foundations of Social Cognitive Theory

Human Agency and Perceived Self-Efficacy

Perceived Self-Efficacy and Task Persistence

Affect and Self-Efficacy

Approaching New Tasks and Sources of Self-Efficacy

Pilot Study of Knowledge of NSSI Behaviors and Related Interventions

Rationale for Current Study

Research Questions and Study Hypotheses

3. Methods

Participants

Instrumentation

Non-Suicidal Self-Injury Survey for School-Based Professionals

Demographic Section

General Knowledge of NSSI Behaviors

Knowledge of Interventions for NSSI Behaviors

Perceived Self-Efficacy

Topical Questions and Comments

Procedures

4. Data Analysis

Research Design 
Multivariate Analysis of Variance (MANOVA) Assumptions

Independence of Observations

Normal Distribution of Dependent Variables

Homogeneity of Covariances and Variances

Linear Regression Assumptions

Normal Distribution of Dependent Variables

Linearity

Interval Level of Variables

Lack of Measurement Error

Homoskedasticity

Lack Multicollinearity

Model Specification

Model Identification

Transformation Procedure to Satisfy Normality Assumption

Missing Data and Unequal Cell Sizes Analysis

A Priori and Post Hoc Power Analysis

Proposed Bivariate Relationship and Analysis

Multivariate Analysis of Variance of Data

Linear Regression and Path Analysis

5. Results

Overall Scores for Respondents on Dependent Variables

Survey Reliability

Multivariate Analysis of Variance (MANOVA) Assumptions

Independence of Observations

Normal Distribution of Dependent Variables

Transformation Procedure to Satisfy Normality Assumptions

Homogeneity of Variance-Covariance Matrices

A priori and Post Hoc Power Analysis for MANOVA

Missing Values Analysis/Unequal Cell Sizes 
Multivariate Analysis of Variance Analysis (MANOVA) Interpretations

Univariate Analysis of Variance

Post Hoc Tests

Direct Experience with NSSI Behaviors

Familiarity with Mental Health Disorders

General Knowledge of NSSI Behaviors

Perceived Self-Efficacy

Bivariate Correlational Data

Perceived Self-Efficacy Bivariate Relationships

General Knowledge of NSSI Behaviors Bivariate Relationships

Direct Experience with NSSI Behaviors Bivariate Relationships

Familiarity with Various Mental Health Disorders Bivariate Relationships

Linear Regressions and Path Analysis Assumptions

Normal Distribution of Dependent Variables

Linearity

Interval Level of Measurement for Dependent Variables

Lack of Measurement Error

Homoskedasticity

Lack of Multicollinearity

Model Specification

Model Identification

Sobel Test of Significant Indirect Effects

Linear Regression and Path Analysis Interpretations

Direct Effects of Predictors on Criterion Variables

Indirect Effects of Predictors on Criterion Variables

Total Effects on Dependent Variables

Absolute Values of Residual

Unanalyzed Model Correlations

Informal Qualitative Analysis

6. Discussion

Implications

Limitations

Future Directions 
$\begin{array}{ll}\text { References } & 164\end{array}$

$\begin{array}{ll}\text { Appendix } & 178\end{array}$

1. Consent Letter for Research Study

2. Study Overview Letter for Schools

3. Email Script for Solicitation of Research Participants

4. Phone/In Person Script for Solicitation of Research Participants

5. Solicitation Cards for School Districts to Participate in Research Study

6. Assessment of School-Based Staff Knowledge and Self-Efficacy Related to NSSI Survey 
Table

\section{LIST OF TABLES}

1. Observed Number of Participants by School-Based Professional's

Role (Total $\mathrm{N}=333$ )

2. Number of Identified School Placements by Grade Level

(Total $\mathrm{N}=333$ )

3. Education Level of School-Based Professionals (Total $\mathrm{N}=332,1$ missing)

4. School-Based Professionals by U.S. Region (Total N = 333)

5. Variables Used to Calculate Path Analysis Coefficients

6. Predicted Directions of Point-Biserial Correlations for Model

7. Range, Mean and Standard Deviation of Dependent Variable Scores by Professional's Role in the School

8. Range, Mean, and Standard Deviation of Dependent Variable Scores by Professional's School Assignment (60\% or more time spent)

9. Range, Mean, and Standard Deviation of Dependent Variable Scores by Professionals' Highest Attained

10. Range, Mean, and Standard Deviation of Dependent Variable Scores by Professionals' Region of the U.S. Worked

11. Multivariate Skewness and Kurtosis Values for Dependent Variables Before Transformation Methods

12. Univariate Test of Skewness and Kurtosis for Role of School-Based Profession by Dependent Variable Before Transformation Methods

13. Multivariate Skewness and Kurtosis Values for Dependent Variables After Transformation Methods

14. Pre and Post Transformation Tests of Normality Values for Questions

Within the General Knowledge of NSSI Behaviors Scale

15. Pre and Post Transformation Tests of Normality Values for Questions Within Professional's Perceived Self-Efficacy Scale 
16. Expected Number of Participants for A Priori Power Analysis of Power $=.95$

17. Missing Value Patterns Among School-Based Professionals for the Knowledge of NSSI Behaviors and Perceived Self-Efficacy Survey Sections

18. Multivariate Analysis: Effects of Role in School and School

19. Univariate Analyses: Effect of School-Based Professional's Role in School on Dependent Variables General Knowledge of NSSI Behaviors, Direct Experience with NSSI Behaviors, Familiarity with Mental Health Disorders, and Perceived Self-Efficacy with Transformed Scores

20. Tukey HSD and Games-Howell Post Hoc Comparison of Mean Scores on Dependent Variables for School Based Professional's Role in the School (After Transformation Methods)

21. Pearson's Point-Biserial Correlations for Independent and Dependent Variables in Path Analysis Model

22. Summary of Mediation Analyses for Direct Experience with NSSI Behaviors and Professionals' Perceived Self-Efficacy Variables by Role of School-Based Professional

23. Summary of Mediation Analyses for Familiarity with Mental Health Disorders Behaviors and Professionals' Perceived Self-Efficacy Variables by Role of School-Based Professional

24. Summary of Mediation Analyses for Direct Experience with NSSI Behaviors and Professionals' General Knowledge of NSSI Behaviors Variables by Role of School-Based Professional

25. Summary of Mediation Analyses for Familiarity with Mental Health Disorders and Professionals' General Knowledge of NSSI Behaviors Variables by Role of School-Based Professional

26. Standardized Direct and Indirect Effects for Role of School-Based Professional on General Knowledge of NSSI Behaviors and Perceived Self-Efficacy 
Figure

\section{LIST OF FIGURES}

1. Path Analysis Model for Special Education Teachers' Perceived Self-Efficacy as Mediated by Direct Experience with NSSI Behaviors

2. Path Analysis Model for School Social Workers' Perceived Self-Efficacy as Mediated by Direct Experience with in NSS Behaviors

3. Path Analysis Model for Support Staff's Perceived Self-Efficacy as Mediated by Direct Experience with NSSI Behaviors

4. Path Analysis Model for School and District Administrators' Perceived Self-Efficacy as Mediated by Direct Experience with NSSI Behaviors

5. Path Analysis Model for School Nurses' Perceived Self-Efficacy as Mediated by Direct Experience with NSSI Behaviors

6. Path Analysis Model for Mental Health and Counseling Associates' Perceived Self-Efficacy as Mediated by Direct Experience with NSSI Behaviors

7. Path Analysis Model for Mental Health and Counseling Associates' Perceived Self-Efficacy as Mediated by Familiarity with Various Mental Health Disorders

8. Path Analysis Model for School Social Workers' Perceived Self-Efficacy as Mediated by Familiarity with Various Mental Health Disorders

9. Path Analysis Model for Support Staff's Perceived Self-Efficacy as Mediated by Familiarity with Various Mental Health Disorders

10. Path Analysis Model for School and District Administrators' Perceived Self-Efficacy as Mediated by Familiarity with Various Mental Health Disorders

11. Path Analysis Model for School Nurses' Perceived Self-Efficacy as Mediated by Familiarity with Various Mental Health Disorders 
12. Path Analysis Model for Special Education Teachers' Perceived Self-Efficacy as Mediated by Familiarity with Various Mental Health Disorders

13. Path Analysis Model for Mental Health and Counseling Associates' General Knowledge of NSSI Behaviors as Mediated by Direct Experience with NSSI Behaviors

14. Path Analysis Model for School Social Workers' General Knowledge of NSSI Behaviors as Mediated by Direct Experience with NSSI Behaviors

15. Path Analysis Model for Support Staff's General Knowledge of NSSI Behaviors as Mediated by Direct Experience with NSSI Behaviors

16. Path Analysis Model for School and District Administrators' General Knowledge of NSSI Behaviors as Mediated by Direct Experience with NSSI Behaviors

17. Path Analysis Model for School Nurses' General Knowledge of NSSI Behaviors as Mediated by Direct Experience with NSSI Behaviors

18. Path Analysis Model for Special Education Teachers' General Knowledge of NSSI Behaviors as Mediated by Direct Experience with NSSI Behaviors

19. Path Analysis Model for Mental Health and Counseling Associates' General Knowledge of NSSI Behaviors as Mediated by Familiarity with Various Mental Health Disorders

20. Path Analysis Model for School Social Workers' General Knowledge of NSSI Behaviors as Mediated by Familiarity with Various Mental Health Disorders

21. Path Analysis Model for Support Staff's General Knowledge of NSSI Behaviors as Mediated by Familiarity with Various Mental Health Disorders 
22. Path Analysis Model for School and District Administrators' General Knowledge of NSSI Behaviors as Mediated by Familiarity with Various Mental Health Disorders

23. Path Analysis Model for School Nurses' General Knowledge of NSSI Behaviors as Mediated by Familiarity with Various Mental Health Disorders

24. Path Analysis Model for Special Education Teachers' General Knowledge of NSSI Behaviors as Mediated by Familiarity with Various Mental Health Disorders 


\begin{abstract}
Non-suicidal self-injurious (NSSI) behaviors are on the rise among school aged children and adolescents. The current research study assessed school-based professionals' general knowledge of NSSI behaviors and perceived self-efficacy in working with students that engage in NSSI behaviors. Direct experience working with students that engage in NSSI behaviors and familiarity with various mental health disorders served as mediators. Familiarity with various mental health disorders served as a significant predictor for most professionals when examining their general knowledge scores and perceived self-efficacy. Direct experience with NSSI behaviors proved to be a weak mediator in the current study. Findings suggest that school-based professionals, overall, do not hold a considerable amount of knowledge regarding NSSI behaviors or confidence in their ability to work with students that engage in NSSI behaviors.

Specific areas for training and interventions are identified based upon study findings. The study emphasizes that school-based professionals are not required nor expected to have all the right answers. However, school-based professionals are encouraged to develop multidisciplinary teams to create action plans that address mental health issues in their schools.
\end{abstract}




\section{CHAPTER ONE \\ INTRODUCTION}

Mental health disorders are a serious and growing public health concern. The number of children and adolescents with one or more diagnosable mental health disorders parallels that of adults. Studies conducted by the National Institute for Health Care Management Foundation and other agencies have indicated that one out of every five children and adolescents or 7.7 to 12.8 million individuals, has a diagnosed or diagnosable mental health disorder (NIHCM Foundation, 2005; National Mental Health Association, 2006). Additionally, recent studies have illustrated that parents of children and adolescents aged 4-17 years old often seek mental health services from a health professional. In 2010, this figure reached $49.3 \%$ of parents seeking services for children with mental concerns (ChildStats.gov, 2012). In addition, 25.7\% of school age youth are currently receiving special education services for serious behavioral and/or emotional disorder (ChildStats.gov, 2012).

These staggering figures illustrate how symptoms and behaviors of various mental health disorders are pervasive in youth populations. The need for evidence-based strategies and informed practices is of the utmost importance to foster overall wellness. The focus of this study is the rising incidence of children and adolescents engaging in non-suicidal self-injurious behaviors that is often indicative of an overarching mental health issue that warrants further attention. 


\section{Spotlight on Non-Suicidal Self- Injurious Behaviors}

Non-suicidal self-injury (NSSI) is defined as the intentional harm of one's body without suicidal intent (Klonsky, Muehlenkamp, Lewis \& Walsh, 2011. Non-suicidal self-injurious (NSSI) behaviors appear to be a common feature serving as a warning sign for major disorders such as depression, anxiety, and eating disorders. With estimates of one out of eight students reporting to have used NSSI as a way to cope (Czarnopys, 2002), it raises the issue of the knowledge base of school-based professionals working with students. While assessment and intervention tools for self-injurious behaviors are available, the question remains whether school-based professionals have the knowledge, skills, and self-efficacy to effectively respond and correctly utilize these tools.

NSSI has been sensationalized in mainstream media outlets and social networking sites such as Facebook and YouTube (Klonsky, Muehlenkamp, Lewis, \& Walsh, 2011). Movies such as Thirteen and Girl, Interrupted depict troubled teenagers struggling to find themselves and coping with daily stressors by cutting their wrists, arms, legs, or other body parts. It is with great concern about this maladaptive coping style that gains the attention of others in these movies. This often mirrors real life for individuals engaging in NSSI behaviors. Non-suicidal self-injurious behaviors often leave the person with permanent scars that serve as physical reminders of emotional pain. Because the behaviors are not a socially sanctioned behavior, engaging in this method of coping is often frowned upon and frightening to others. These responses often leave the person feeling isolated and troubled resulting in withdrawal and continued use of NSSI behaviors to cope (Klonsky, Muehlenkamp, Lewis \& Walsh, 2011; Nock \& Cha, 2009). 
From a clinical standpoint, NSSI behaviors may be difficult to treat. Reasons beyond the covert nature of the behavior baffle clinicians and other professionals attempting to diagnose and intervene with individuals. This subset of individuals often has comorbid disorders that can go untreated as well as deficit in skills and strategies that enable them to manage stressors more adaptively. Moreover, the terminology and classification of NSSI is riddled with diagnostic criteria that are inconsistently utilized by professionals leading to ineffective outcomes for individuals that engage in NSSI behaviors.

\section{Non-Suicidal Self-Injury and Mental Health Training of School-Based Professionals}

The role of differentiated instruction and behavioral interventions have been typically developed and implemented by special educators, school psychologists, and counselors trained to provide additional supports to students struggling with curriculum demands and mental health issues (Gilman \& Medway, 2007; Youngs, Jones, \& Low, 2011). Since a Free and Appropriate Education (FAPE) is a right provided through the Individuals with Disability Education Improvement Act (IDEIA, 2004), mainstreaming students who are in need of additional supports was made a priority. Consequently, educators viewed other school-based professionals (such as school psychologists and school counselors) as vital interventionists and deliverers of mental health services in schools (Reinke, Stormont, Herman, Puri, \& Goel, 2011). However, it is the collaborative effort of all school-based professionals that may prove advantageous in meeting the needs of students, particularly those with emotional and behavioral issues.

A number of evidenced-based programs that address social emotional and 
behavioral concerns are increasingly implemented in schools. These programs strive to improve academic and social emotional functioning while creating safe learning environments. At the pre-service level, due to increasing mental health concerns, it is not surprising that graduate programs are incorporating mental health courses in their training curriculum for regular and special education teachers (MPER, 2009). Pre-service and inservice trainers are recognizing that children do not exist within a vacuum, but are influenced in significant ways by their home, neighborhood, and school environments as well as their genetic makeup. Related programs such as those seeking to prevent suicide are easily visible in schools given obvious concerns over this problem. Schools are looking to evidence-based prevention strategies to curb the incidence of this rising concern (Miller \& Brock, 2010).

\section{Applications of Non-Suicidal Self-Injury Knowledge for School-Based Professionals}

School-based professionals are on the frontlines of identifying and assisting individuals with mental health issues. Students may present with a number of mental health concerns that manifest themselves through behavioral or emotional regulation difficulties. Possessing knowledge of and having direct experience with mental health disorders and features such as non-suicidal self-injury could greatly influence the selfefficacy of helping professionals (Lieberman, Toste, \& Heath, 2009). For example, school-based professionals could work individually with students to target the distorted thinking of individuals who engage in self-injurious behaviors to counter feelings of inadequacy, loss, rejection, and fear (Alderman, 1997; Glassman, Weierich, Hooley, 
Deliberto, \& Nock, 2007; Lieberman, Toste, \& Heath, 2009; Lloyd-Richardson, Perrine, Dierker, \& Kelley, 2007).

Unfortunately, there are many myths and misconceptions about self-injury and individuals engage in this behavior, which can lead to increased alienation and ineffective treatments (Alderman, 1997; Conterio \& Lader, 1998; Klonsky, Muehlenkamp, Lewis \& Walsh, 2011; Miller \& Brock, 2010; Schinagle, 2002). These myths and misconceptions stem from a lack of knowledge of mental health disorders in general and drivers of NSSI behaviors in particular. With these critical elements in mind, it is important to clearly define and understand self-injury with respect to various clinical and general populations, particularly school-based populations. Moreover, there is also a need to understand the development and implementation of prevention and intervention strategies in schools to address this growing concern among adolescents (Lieberman, Toste, \& Heath, 2009; Miller \& Brock, 2010).

Previous study findings suggest that most school-based health and mental health professionals, overall, do not hold a high level of knowledge of NSSI behaviors (Best, 2005; Heath, Toste, Sornberger, \& Wagner, 2011; Moore, 2009). The purpose of this study is to investigate the level of self-efficacy of school-based professionals who are currently working or have worked with students who engage in NSSI behaviors. Additionally, the study intends to ascertain school-based professionals' knowledge of NSSI and related interventions. The methodology employed in the current study will assess these outcome variables using school-based professional's direct experiences in working with students who engage in NSSI behaviors and their overall familiarity with mental health disorders as a mediators. Inquiry into these areas is extremely timely and 
relevant as all school-based professionals share a role in creating a safe school environment for students (Lieberman, Toste, \& Heath, 2009).

The literature review will provide a comprehensive definition of non-suicidal selfinjury; relationship between suicide and non-suicidal self-injury; characteristics of individuals engaging in NSSI behaviors; assessment techniques of non-suicidal selfinjury; treatment options; and an overview of Social Cognitive Theory (SCT) for its applicability to school-based staff's self-efficacy in working with students that engage in NSSI behaviors. The literature review is followed by a discussion of specific research questions and their relevance to the current study. Hypotheses are presented based on the literature review, SCT theory and pilot study findings (Moore, 2009). Data analysis includes multivariate analysis of variance (MANOVA) for within- and between-group comparisons of self-efficacy and general NSSI knowledge; reliability calculations for the developed measure; and a path analysis to explore causal relationships among variables in mediation models. Lastly, informal qualitative information offered through additional comments from participants regarding mental health, NSSI behaviors, and/or training will be summarized. The writing concludes with a discussion of the limitations of the study and future implications. 


\section{CHAPTER TWO \\ LITERATURE REVIEW}

\section{Defining Non-Suicidal Self-Injury}

It is important to precisely define and correctly identify self-injurious behaviors in youth to better inform prevention and intervention practices. The literature offers a number of definitions that focus on the functionality and intentionality of self-injurious behaviors, in general, and NSSI behaviors, specifically. Functionality refers to why the individual is engaging in self-injurious behaviors while intentionality identifies whether the self-injurious behavior is in fact a willful and purposeful act to serve a particular purpose (Nock \& Cha, 2009). A. R. Favazza (1987) coined the term “self-mutilation" to describe self-injurious behaviors. According to Favazza (1987), there are three categories of self-mutilation that consist of a range of behaviors. They are stereotypic, major, and moderate or superficial. Stereotypic behaviors are those behaviors that are generally associated with individuals with pervasive developmental disorders such as autism or mental retardation, and may include such actions as intentional head banging, throwing their bodies against walls and/or corners of tables (Favazza, 1987).

Major self-mutilation is the rarest form of self-injury (Alderman, 1997; Favazza, 1987; Stirn \& Hinz, 2008). Behaviors associated with major self-mutilation include rites of passage, tribal rituals, genital mutilation, amputations, and castrations (American Academy of Child and Adolescent Psychiatry, 1999). Favazza (1987) further explains that these ritualistic behaviors often are socially sanctioned, and that within one's own culture, the act of mutilation is acceptable (Stirn \& Hinz, 2008). Examples more common to Western culture may include excessive plastic surgery, numerous tattoos, and multiple 
body piercings (Alderman, 1997; Favazza, 1987; Levenkron, 1998; Nock et al., 2006). These behaviors are excluded from the milder forms of self-injury because an outside party typically performs the injurious behaviors. Additionally, the intent or goals that direct these behaviors are completely contradictory to most moderate or superficial selfinjurers.

Self-injury, referred to as superficial or moderate self-mutilation (Favazza, 1987), is defined as the intentional harm of one's own body without conscious suicidal intent (Alderman, 1997; Claes \& Vandereycken, 2007; Favazza, 1987). Other terms such as cutting, parasuicide, non-suicidal self-injury, self-harm, and self-mutilation are also seen throughout the literature and refer to similar behaviors (Nixon \& Heath, 2009; Stirn \& Hinz, 2008). Recent clinical studies are emerging that make even finer distinctions between self-injury and self-harm (Claes \& Vandereycken, 2007). These researchers argue that clarity is needed when using terminology that describes various self-injurious behaviors. If this is not done, then the body of self-injury literature is muddled through the proliferation of absent or imprecise definition. Claes and Vandereycken also support this with several studies that describe the same type of behavior and provide either a functional or clinical/medical explanation for the behavior, yet have used terms such as self-mutilation, self-harm, self-injury, self-damage, and self-aggression.

As a result, Claes and Vandereycken (2007) propose a diagnostic system to assist professionals in determining whether behaviors are non-suicidal self-injury, self-injury (e.g., suicide attempts) or an overarching clinical disorder (i.e., factious disorder, eating disorder). To illustrate further, Lloyd-Richardson, Nock and Prinstein (2009) offer a four factor model of NSSI used to determine the function of the behavior and further delineate 
the construct. The model is based on behavior principles such as reinforcement and contingencies that explain how the behavior is initiated and maintained.

Some researchers argue for the sole use of the term "self-injury" to describe behaviors. They reason this terminology is more appropriate in capturing the essence of the underlying intent (Adler \& Adler, 2007). For example, individuals who engage in NSSI behaviors lack evidence of suicidal ideations, suicidal intent, and bodily disfiguration (Alderman, 1997; Claes \& Vandereycken, 2007; Levenkron, 1998). However, some researchers have found that those who engage in body modification practices share common characteristics with those who engage in NSSI, such as sensation seeking and presence of social stressors (Stirn \& Hinz, 2008). Nonetheless, Alder and Alder (2007) posit that self-injury conveys sensitivity to the individual engaging in selfinjurious behaviors and is the most appropriate descriptor.

According to the American Academy of Child and Adolescent Psychiatry (AACAP) (1999), NSSI behaviors include the following: carving, scratching, branding, marking, picking and pulling skin and hair, burning/abrasions, biting, excessive nail biting, bruising, hitting, and cutting using razors, scissors or other sharp objects. Studies in psychology and psychiatry have attempted to define and examine abnormal behavior, which has resulted in a comprehensive diagnostic system that provides specific criteria for diagnoses (Diagnostic and Statistical Manual-IV-Text Revisions abbreviated DSMIV-TR; American Psychiatric Association, 2000).

Despite these efforts, self-injury is not recognized by the DSM-IV-TR (2000) as a distinct clinical disorder. Rather, it remains a feature or manifestation of underlying disorders such as Borderline Personality Disorder, Eating Disorders, Tic Disorders, Depression, 
factitious disorders and stereotypic disorders (Deliberto \& Nock, 2008; Lloyd-

Richardson, Perrine, Dierker, \& Kelley, 2007; Lofthouse, Muehlenkamp, \& Adler, 2009).

The definition of NSSI remains a controversial issue. A number of researchers

propose a multi-functional approach to examining how abnormal behavior develops and is maintained due to their varying etiology (Wenar \& Kerig, 2006). For example, when examining the common factors related to individuals engaging in self-injurious behaviors, there is often a history of abuse (Lloyd-Richardson et al., 2007; Nock et al., 2006), poor childhood attachment to caregivers (Lloyd-Richardson et al., 2007; Nock et al., 2006; Nock \& Mendes, 2008; Walsh, 2005) and dichotomous, "all or nothing" thinking as adolescents and young adults (Nock et al., 2006). "All or nothing" thinking often leaves the individual little ability to reason beyond two options--perfection or the most disastrous situation imaginable. This suggests that self-injury, more specifically non-suicidal self-injury, can be defined and classified according to various behaviors and thought processes communicated by the individual.

\section{Distinguishing Features of Non-Suicidal Self-Injury}

There are several defining features that further distinguish NSSI behaviors from other types of self-harm behaviors. Three factors should be considered when determining the degree to which an individual engages in self-injurious behaviors. These are directness, lethality, and repetition. Directness refers to the intentionality of the act or behavior (Kahan \& Pattison, 1984). Assessing the individual's objective in producing harm to themselves is vital in determining if there is a conscious intent to produce harm. This distinction typically refers to individuals contemplating suicide. Lethality refers to 
the likelihood that death occurs from engaging in the behaviors in the immediate or near future (Kahan \& Pattison, 1984). It is important to assess if the behavior was intended to seriously harm the individual to the extent to which death is probable. Lastly, repetition refers to whether the act or behavior is repeated at some frequency over a period of time (Kahan \& Pattison, 1984). All of the factors fall along a continuum and there are specific behaviors that are associated with varying degrees of self-injurious behaviors. With respect to non-suicidal self-injury, the behavior is direct with an intended low level of lethality and high occurrence of repetition.

\section{Non-Suicidal Self-Injury Prevalence}

As estimated from community samples and clinical samples, respectively, approximately $4 \%$ of the adult population and $12-21 \%$ of children and adolescents have a history of engaging in NSSI behaviors (Deliberto \& Nock, 2008; Glassman, Weierich, Hooley, Deliberto, \& Nock, 2007; Lloyd-Richardson, Perrine, Dierker, \& Kelley, 2007). In clinical populations, this statistic ranges from $21-65 \%$ for children and adults (see Heath, Schaub, Holly, \& Nixon, 2009 for a detailed prevalence review). Prevalence figures vary widely by the definition of self-injurious behaviors, their setting, as well as the reported time frame in which the behaviors occur. In one sample, for example, the researchers included suicide attempts as they identified $17 \%$ of adolescents engaged in NSSI behaviors (Jacobson, Muehlenkamp, Miller, \& Turner, 2008). In another sample, $39 \%$ of adolescents admitted to only NSSI behaviors (such as cutting, burning, and/or scratching) in the past year (Lloyd, Kelley, \& Hope, 1997). When parsing out individuals that have engaged in NSSI behaviors in their lifetime, the prevalence falls within a range 
of $12 \%-58 \%$ across diverse settings (e.g., hospital treatment centers, high schools, etc.; Heath, Schaub, Holly, \& Nixon, 2009).

\section{Characteristics of Individuals who Engage in Non-Suicidal Self-Injurious Behaviors}

Although there are many common characteristics among individuals who engage in NSSI behaviors, it is important to note that not all individuals fall neatly within particular categories. The specific population can be circumscribed depending on the presenting sample. With empirical data, researchers have found the most common characteristics among individuals that engage in non-suicidal behaviors are the presence of a psychiatric disorders, age, and gender (Adler \& Adler, 2007; Czarnopys, 2002; Jacobson et al., 2008; Nock et al., 2006). Other factors such as SES and race/ethnicity have been reported and examined across various studies as well (Klonsky et al., 2011). These characteristics are further defined and elaborated in the following sections.

Psychiatric disorders. According to Conterio and Lader (1998) self-injurious behaviors occur within a variety of clinical populations. These clinical populations include individuals with borderline personality disorder, obsessive-compulsive disorder, bipolar disorder, major depressive disorder, dissociative disorder, eating disorders, and posttraumatic stress disorder (PTSD; Alderman, 1997; Conterio \& Lader, 1998; Jacobson et al., 2008; Klonsky et al., 2011; Levenkron, 1998). Most documented cases of selfinjury are in individuals who have a diagnosis of borderline personality disorder (Alderman, 1997; Conterio \& Lader, 1998; Levenkron, 1998; Linehan, 1993). Rollink et al. (2001) report that self-injury can be observed in up to 50 percent of individuals with borderline personality disorder. These individuals typically feel misunderstood and 
frustrated (Conterio \& Lader, 1998; Levenkron, 1998), and may assume that therapists are frightened of them because of their behavior (Levenkron, 1998).

The most common correlate with self-injury is a history of sexual abuse (Zoroglu et al., 2002). These individuals typically receive a diagnosis under Post Traumatic Stress Disorder (PTSD), which is an Axis I disorder rather than the self-injury being the primary diagnosis. Other researchers have examined the relationship between self-injury and substance abuse (Israel \& Lee, 2002; Waska, 1998). These researchers note that there may in fact be a stronger correlation between self-injury and substance abuse than is reported. As with every case with self-injury, and more specifically non-suicidal selfinjury, it remains a feature of an Axis I or Axis II disorder such as substance abuse or borderline personality, respectively (Adler \& Adler, 2007; Jacobson et al, 2008).

Although clinical disorders and related symptoms are frequently associated with individuals that engage in non-suicidal self-injurious behaviors, there are subgroups of individuals that have little to no clinical symptoms who engage in the behavior as well (Klonsky \& Olino, 2008). The subgroups are further classified by the frequency in which the individuals engage in NSSI behaviors and its onset. For example, individuals who engage in NSSI behaviors occasionally may have a late onset and experimented with the NSSI while other subgroups with an early onset of NSSI and more severe clinical symptoms engage in the NSSI more frequently and have a history of suicidal ideation and attempts. Klonsky and Olino (2008) found a significant relationship between severity of non-suicidal self-injurious behaviors and clinical symptoms further supporting the assertion that self-injurious behaviors are on a continuum and treatment should be 
individualized to address specific thoughts and behaviors exhibited by various individuals (Kahan \& Pattison, 1984; Klonsky et al., 2011; Klonsky and Olino, 2008).

Age. Research has shown that self-injury, generally, first appears in the individual's mid-teens, peaks in their early- to mid-20s and decreases in their 30s (Alderman, 1997; Heath et al., 2006; Lloyd-Richardson et al., 2007). Other researchers have further supported this assertion by noting that the period of early adolescent is riddled with hormonal, body, and life changes. Alderman (1997) also states that selfinjury as a coping style becomes less effective as the individual gets older which causes the individual to search for more effective ways in dealing with stress. Older individuals that continue to rely on self-injury as a coping mechanism often feel isolated and out of place. Additionally, they are more likely to engage in other forms of self-harm, such as suicide or attempted suicide (Adler \& Adler, 2007; Jacobson et al, 2008). Recent findings have suggested that the presence of NSSI behaviors in childhood should not be overlooked. For example, a study asked individuals currently engaging in NSSI behaviors to report the age that began NSSI behaviors. Some individuals reported they engaged in NSSI behaviors as early as four years of age (Klonsky et al., 2011).

Gender. Favazza and Conterio (1989) conducted a survey that typed a typical individual engaging in NSSI behaviors (see Czarnopys, 2002). Their research found that females in their teens to mid-20's are more likely to engage in NSSI behaviors. However, other researchers posit that NSSI behaviors are also observed in male populations (Yates, Tracy \& Luthar, 2008). According to White, Leggett, and Beech (1999), there are a significant percentage of males who engage in self-injurious behaviors; however, it tends to be overshadowed because females are more likely to seek treatment for their 
maladaptive behaviors. In fact, the study found striking similarities between how males and females injure themselves as well as past experiences (e.g., sexual abuse and physical abuse) that contribute to an individual using self-injury as a coping mechanism (Ross \& Heath, 2002; White et al., 1999). Additionally, males typically use more violent modes of self-injurious behaviors and suicide than females, suggesting the importance of equal recognition of self-injurious behaviors in this population (Hawton, 2000).

Race and Ethnicity. The literature is filled with mixed results regarding the relationship between race and ethnicity and NSSI behaviors. Various studies found white American adolescents and young adults engage in NSSI behaviors more than any other race or ethnicity (Nixon, Cloutier, \& Jansson, 2008; Nock \& Prinstein, 2004; Ross \& Heath, 2002; Newhill, Eack, \& Conner, 2009). Conversely, several studies across the world, including Wales, Turkey, and Japan, have identified self-injury within their population of young adults (Matsumoto, et al., 2004; White et al., 1999; Zoroglu, et al., 2002). Further, an international study found Native American and Latino adolescents engaged in the behavior more than white American and African-American adolescents (Evans, Evans, Morgan, Hayward, \& Gunnell, 2005). Yet another study of adolescents in an urban, clinical population found African American and Latino youth engaged in NSSI at similar rates as their white American counterparts (Jacobsen, Muehlenkamp, Miller, \& Turner, 2008). Ultimately, the population sampled and the methods used to assess NSSI will determine the ethnic "majority" in which NSSI behavior is found (Gratz, 2006; Jacobson et al., 2008; Klonsky et al., 2011; Ross \& Heath, 2002).

Socioeconomic Status. The evidence is equivocal when examining the relationship between socioeconomic status and NSSI. Most studies have been conducted 
with individuals who engage in NSSI behaviors in wealthy countries as they survey suburban and urban areas in the US (Ross \& Heath, 2002; Yates, Tracy, \& Luthar, 2008; Zila \& Kiselica, 2001). In a recent cross-national study, Nock et al. (2008) found little research exists on self-injurious behaviors in third world countries, but still warranted examination as a comparison to incidence rates of NSSI in the United States. Nock (2008) found the occurrence of NSSI existed greatly in wealthier countries when compared to less developed countries. It is unclear whether SES plays an important discriminatory role for individuals who engage in self-injurious behaviors in the United States and other developed nations.

\section{Similarities and Differences between Suicide and Non-Suicidal Self-Injury}

Suicide is one of the leading causes of death for children and adolescents (Nock, Joiner Jr., Gordon, Lloyd-Richardson, \& Prinstein, 2006). The Youth Risk Behavior Surveillance (YRBS) has identified students as young as 10 years of age as having suicidal ideation or attempted suicide within the last 13 months (Center for Disease Control and Prevention, 2007).

There are various distinct characteristics within the construct of suicidal selfinjurious behaviors. For example, suicide attempt refers to self-injurious behaviors where death is intended. Suicidal gesture refers to behaviors, both verbal and non-verbal, that imply an individual is thinking of making a suicide attempt, but the intention to follow through with the attempt is not present (Nock, 2008). This is an important distinction that serves as an assessment tool to determine lethality of self-harming behaviors. Although motives behind engaging in suicidal behaviors and gestures may vary, they are, 
nonetheless, an indication of underlying problems insofar the individual is seeking help and attention from others.

Commonalities between suicide and non-suicidal self-injurious behaviors are vast and adversely affect children and adolescent behavior during critical period of development (Prinstein, Nock, Simon, Aikins, Cheah, \& Spirito, 2008). For example, negotiating peer influence and social circles, academic concerns, family issues, and physical growth and development can impact the student's wellbeing. Emotional development may also adversely impact students' functioning. Related to emotional development and functioning is impulsivity, which has been found to be a common factor between suicide and non-suicidal self-injury-especially when the student is under duress (Jacobson et al., 2008; Klonsky et al., 2011; Nock et al., 2006). Nonetheless, suicide and non-suicidal self-injury remains a muddled area of research because of ambiguities in the intent that drives each act (Jacobson, et al., 2008).

\section{Assessment of Non-Suicidal Self-Injurious Behaviors}

There are a number of measures that are used to assess potential and current NSSI thoughts and behaviors. Among those previously developed are comprehensive health and mental health measures and/or manualized treatments programs/modules such as the Youth Risk Behavior Survey (YRBS), the Behavior Assessment Scales for Children (BASC), and the Diagnostic Interview Schedule for Children eating disorders module (see Hilt, Lloyd Richardson, \& Prinstein, 2008; see Klonsky \& Olino, 2008). Other specific measures are targeted to overarching psychiatric disorders for which self-injury is a feature such as depression, anxiety and borderline personality (see Hilt et al., 2008; 
see Klonsky \& Olino, 2008). For example, some of these measures include the McLean Screening Instrument for Borderline Personality Disorder, the Depression and Anxiety Stress Scales, and the Diagnostic Interview Schedule for Children (DISC; see Klonsky \& Olino, 2008; see Lloyd-Richardson et al., 2007; see Nock et al., 2006; see Nock et al., 2007).

There are few empirically validated, comprehensive measures that solely focus on the specific behavior of NSSI (Nock et al., 2007). These instruments measure aspects of NSSI such as the age of onset, frequency, intensity, duration, and function of selfinjurious behavior. Some examples include the Self-Injurious Thoughts and Behaviors Interview (SITBI), the Lifetime Parasuicide Count (LPC), and the Functional Assessment of Self-Mutilation (FASM). These measures are in interview format and have been shown to have acceptable reliability (Jacobson, 2008; Lloyd-Richardson et al., 2007; Nock et al., 2007). Other measures have been specifically designed for the study by the research and reportedly have face validity. For instance, a question from a specific measure designed by Hilt et al. (2008) to assess self-injurious behaviors in their study was, "Have you harmed or hurt your body on purpose (for example, cutting or burning your skin, hitting yourself, or pulling out your hair)?"

Factors that are typically associated with NSSI behaviors have also been assessed. Because individuals who engage in NSSI behaviors may also have a history of familial or peer relationship problems, Hilt et al (2008) incorporated variables to examine these factors in The Inventory of Parent and Peer Attachment (IPPA). Additionally, students may have a history of suicide; therefore, it is not uncommon for a measure of suicidal ideation or suicidality to appear in a standard battery of NSSI behavior assessment. 
Clearly, there are a wide variety of assessment measures for assessing nonsuicidal self-injurious behaviors. These measures gather information through a self-report checklist or interview format. From a practical standpoint, there is a shortage of empirically validated, user-friendly and quick measures for screening or assessments in non-clinical settings such as schools.

\section{Treatment Options for Individuals who Engage in Self-Injurious Behaviors}

A number of effective evidence-based interventions are available in reducing the occurrence of NSSI behaviors. Once barriers, such as the reluctance to seek treatment have been addressed, professionals can begin the therapeutic process. NSSI interventions fall in two categories: behavioral and pharmacological. Behavioral strategies that impart alternate coping skills for individuals have been widely accepted as the most effective treatment for NSSI behaviors (Nixon, Aulakh, Townsend, \& Atherton, 2009). Pharmacological options are less conclusive and more experimental in addressing NSSI behaviors, specifically. Often overarching mental health issues dictate which medications are prescribed (Bloom \& Holly, 2011).

Behavioral Therapy. Many psychotherapists suggest a regiment of medication and behavioral interventions as an effective treatment plan addressing NSSI behaviors (Rollink et al., 2001). For example, Dialectical Behavioral Therapy (DBT) has proven to be a highly effective cognitive-behavioral treatment for individuals with borderline personality in which NSSI behaviors are typically an underlying feature (Bohus, Haaf, Stiglmayr, Pohl, Bohme, \& Linehan, 2000; Schinagle, 2002; Share Shame, n.d.). DBT entails combining cognitive behavior therapy with social skills training 
(Linehan, 1993). The focus is not on finding the solution, but rather clearly breaking down the problem in order to define it and equip the individual with the necessary skills to address the problem (Schinagle, 2002). The primary assumptions for individuals using DBT are that individuals are suffering from emotional dysregulation and absolute thinking (i.e., thinking in black or white terms) (Schinagle, 2002). With respect to NSSI, it is assumed that individuals are engaging in the behavior in response to various stressors or environmental stimuli. The four stages of DBT focus on decreasing life threatening behaviors and posttraumatic stress symptoms while increasing the individual's self-worth and quality of life (Linehan, 1993; Miller, Rathus, \& Linehan, 2006; Schinagle, 2002). Lastly, a treatment plan that focuses on affect regulation, communication and control is recommended to address the variety of underlying causes of individuals who engage in self-injurious behaviors. (Kirkcaldy, Brown, \& Siefen, 2007; Linehan, 1993).

Psychopharmacological options. Neurological deficits such as low levels of neurotransmitters (e.g., serotonin,) and defective receptor sites may be present in individuals who engage in self-injurious behaviors (see Rollinik et al., 2001; Share Shame, n.d.). These findings suggest that individuals who engage in self-injurious behaviors have ineffective mechanisms or low levels of the neurochemicals that normally help to alleviate feelings of inadequacy associated with mental health disorders.

Consequently, Rollink et al. (2001) suggest psychotropic medicines in therapeutic treatment plans for those who engage in NSSI behaviors. In addition to treatments that are behavioral in nature, medications such as opioid antagonists and neuroleptics (e.g., clozapine, risperidone) are recommended (Rollink et al., 2001). Selective serotonin reuptake inhibitors (SSRIs) have mixed results when used to address NSSI behaviors in 
individuals rather than specific mental health disorder(s) that may be present (Bloom \& Holly, 2011). Opioid antagonists (e.g., naltrexone) affect the release of endorphins of those who engage in self-injurious behaviors while neuroleptics help treat psychosis. Cassano et al. (2001) suggest that antidepressants and mood stabilizers act as effective medications in addressing self-injuring behaviors. It should be noted that for both studies cited above, patients were diagnosed with borderline personality disorder (BPD) and not solely NSSI behaviors. Though promising, there are no long-term studies to demonstrate these interventions are efficacious in extinguishing the occurrence of NSSI behaviors.

School-Based Interventions. There are a number of empirically validated interventions that have been presented in the literature to assist students who engage in self-injurious behaviors in schools. Strategies include: creating a safe environment for the student within the school; providing structure and support within the school community; helping students take responsibility for their behaviors; teaching students more adaptive ways to cope with stressors in their lives; involving other agencies to create interdisciplinary and interagency teams within the school to support students; identifying triggers or cues that prompt the behavior; identifying more positive coping strategies; identifying safe people and safe places for students to go; reducing harm that arises from the behavior; avoiding shaming and startling reactions when treating a student who engages in NSSI behaviors; and, developing a protocol for detection, intervention, and referral (D’Onofrio, 2007; Miller \& Brock, 2010; Nixon \& Heath, 2009; Shapiro, 2008; Walsh, 2005). Because these strategies involve both health and mental health aspects, it is incumbent upon school-based professionals to work collaboratively to address this growing concern. 


\section{Variables Associated with Non-Suicidal Self-Injurious Behaviors}

Individuals who engage in NSSI behaviors do so as a coping mechanism for acute and chronic stressors (Klonsky \& Weinberg, 2009; Lloyd-Richardson, Nock, \& Prinstein, 2009; Lloyd-Richardson, Perrine, Dierker, \& Kelley, 2007). Research suggests that individuals engaging in NSSI behaviors often feel remorseful for previous events that have happened in their lives (Klonsky et al., 2011; Lloyd-Richardson, Nock \& Prinstein, 2009). These may include sexual and/or physical abuse, or family issues such as divorce (Deliberto \& Nock, 2008; Kirkcaldy, Brown, \& Siefen, 2007). Moreover, individuals that engage in NSSI behaviors are often self-critical and suffer from feelings of inadequacy (Jacobson et al., 2008). Also, environmental factors may illustrate a moderating relationship that may demonstrate varying degrees of an individual engaging in NSSI behaviors (Lloyd-Richardson, Nock, \& Prinstein, 2009). The environment in which the individual engages in NSSI could be a mediating and/or moderating factor. For example, if individuals have a home environment that is strife-ridden, it may be more difficult to cope with various physical or emotional events and thus engage in NSSI behaviors. On the other hand, buffering factors such as caring adults, extracurricular activities, and other adaptive coping strategies may preclude an individual with a troubling environment from engaging in NSSI behaviors. Consequently, researchers have wrestled with pinpointing one particular reason as to why individuals engage in NSSI, which is the center of debate to date (Lloyd-Richardson, Nock, \& Prinstein, 2009). It is likely there are multiple drivers across multiple systems that maintain NSSI behaviors and other symptoms related to overarching mental health disorders. 
Peer associations may be another strong moderating and/or significant mediating variable to determine if individuals use NSSI to cope with daily life stressors. Findings suggest that when individuals are exposed to or associate with others engaging in NSSI, they are more likely to engage in NSSI as well (Claes, Houben, Vandereycken, Bijttebier, \& Muehlenkamp, 2010; Nock \& Prinstein, 2005). Although there may be a predisposition for mental health disorders such as depression or anxiety for individuals engaging in NSSI, they feel as if NSSI may be the best coping mechanism for them when their peers view it as effective. These findings are supported by the increasing number of individuals reporting that NSSI facilitates a cathartic release of emotions via social media and networking sites such as YouTube (Klonsky et al., 2011; Lewis, Heath, St. Denis, \& Noble, 2011). It was observed that videos that illustrated an individual or fictional character engaging in NSSI behaviors received the greatest number of hits on YouTube (Lewis, Heath, St. Denis, \& Noble, 2011).

\section{Non-Suicidal Self-Injury Through an Ecological Orientation Lens}

From an ecological standpoint, mental health disorders can adversely affect functioning within various social systems (e.g., family, school, community). Similarly, these subsystems can serve as maintaining antecedents or consequences for individuals' maladaptive behaviors. It is estimated up to $7 \%$ of children diagnosed with a mental health disorder are not receiving the care that they so desperately need (NIHCM Foundation, 2005). Access to affordable and quality care is one of many reasons that practitioners are looking at other resources to address this growing concern. 
This may involve incorporating strengths and positive connections across all environments to address symptomatic behaviors. It also entails addressing specific stressors across environments. For example, when examining the family subsystem, often invalidating comments, traumatic events and poor boundaries add to the development of symptoms associated with various mental health disorders (Henggeler, Schoenwalk, Borduin, Rowland, \& Cunnigham, 2009). Often, the aforementioned risk factors may leave the individual searching for effective coping mechanisms that are often maladaptive such as NSSI. What remains a key factor for the home environment is the potential supportive and caring attitudes that counter the individual's inability to regulate their emotions effectively. Huey et al. (2004) conducted a study that examined the effects of working with families and other subsystems by randomly assigning individuals to Multisystemic Family Therapy (MST) or inpatient treatment. The study found symptoms associated with various mental health disorders were significantly reduced with MST, but results should be viewed with caution given that almost half of these individuals also received inpatient treatment.

Community, which may refer to neighborhoods and other social environments can also maintain or buffer the symptoms of mental health disorders such as NSSI. For example, some neighborhoods may be nestled in pockets of urban areas or located in rural areas that lack the resources to support individuals and families experience mental health crises. As a result, schools are increasingly becoming one ideal choice to provide preventative and early intervention care to children and adolescents (Best, 2005; Moor et al., 2007). Since most children and adolescents spend a significant amount of time in 
schools, they serve as an prime locale to address mental health issues that may not ordinarily be addressed elsewhere in the community.

While it is widely accepted that students attend school to learn, it is important to note the processes and optimal situations in which learning occurs. School-based professionals are in a unique position to address these issues in the environment in which learning occurs. The multiplicative effect of all subsystems in which the individual exists are influential and should be taken into consideration when developing effective interventions addressing mental health disorders.

\section{Community and School-Based Health and Mental Health Professionals Knowledge of NSSI Behaviors}

Various studies examining the perceptions of teachers, health professionals, hospital staff and psychologists (Best, 2005; Heath, Toste, \& Beetam, 2006; Huband \& Tatum, 2000) were conducted to determine their readiness in working with individuals engaging in NSSI behaviors. Findings showed no school- or community-based professional was neither exceptionally knowledgeable nor comfortable working with individuals that engage in NSSI behaviors. Furthermore, Huband and Tatum (2000) found that subtle and/or complex aspects of NSSI, such as how to differentiate self-injury from suicidal gestures or copycat cutting, are even more likely to be misunderstood. It should be noted that when teachers have experience with individuals engaging in NSSI, awareness significantly increased (Best, 2005).

Additionally, evidence-based interventions that are practical have been found in a number of studies (Best, 2005; Huband \& Tatum, 2000; Lieberman, Toste, \& Heath, 
2009). According to the mean scores obtained by participants in Huband and Tatum's (2000) study, there were professionals who indicated they were unsure or would never implement some of the empirically validated interventions. Participants were also more likely to endorse "Always" to "With my current knowledge, I would immediately refer this student to a better qualified person such as a psychiatrist to deal with this issue.” This was further confirmed by the informal qualitative analysis with $23 \%$ of individuals who offered suggestions and comments indicated they would refer to an outside person or agency. This is also supported by the current literature that found clinical staff (e.g., psychiatric nurses, psychologists, and non-psychiatric nurses) in need of more evidencebased interventions (Huband \& Tatum, 2000). Again, these findings come from a professional mental health population in which accurate knowledge and practice of evidence-based interventions with individuals who engage in self-injurious behaviors would be expected.

Equally important are opportunities for inservice training or professional development for school-based professionals involving NSSI behaviors. Despite the readily available material through organizations such as the American Psychological Association (APA), National Association of School Psychologists (NASP), Cornell University, Mayo Clinic, and Self Abuse Finally Ends (S.A.F.E.) Alternatives, it is surprising that school-based health and mental health professionals have little to no knowledge at all (Best, 2005; Moore, 2009). These individuals are professionals that serve as resources for other school-based staff and students. For instance, S.A.F.E. Alternatives offers training to organizations looking to create groups assisting individuals who engage in NSSI behaviors. Additionally, S.A.F.E. Alternatives offers inpatient and 
outpatient services to individuals engaging in NSSI behaviors. NASP offers public domain podcasts and written materials to assist school-based professionals in effectively working with students that engage in NSSI behaviors. These organizations in conjunction with the proliferation of scholarly articles and texts, present a plethora of information and evidenced-based interventions for NSSI behaviors.

Even more telling are the low scores obtained in the "capacity to provide interventions section' of the survey. This refers to the access to resources and their selfefficacy in providing interventions to individuals who engage in self-injurious behaviors. Findings were consistent with the literature examining both school-based (e.g. counselors, school nurses and teachers) and clinical mental health staff (Best, 2005; Crawford, Geraghty, Street, \& Simonoff, 2003; Heath, Toste, \& Beetam, 2006; Huband $\&$ Tatum, 2000). It is important for school-based health and mental health professionals to have access to resources not only outside of the school, but also within the school (Best, 2005).

In fact, literature suggests NSSI behaviors are increasingly happening within schools and that access to evidence-based interventions and supports from other schoolbased professionals are paramount. These ideas are related to the evidence-based interventions shared in previous sections. General knowledge of self-injurious behaviors, knowledge of interventions, and having the capacity to provide those interventions are interrelated. School-based health and mental health professionals should possess skills needed to meet the needs of the growing population of students engaging in NSSI behaviors. For training purposes, it is vital to any successful intervention that deficits in knowledge and skills are identified. Once appropriately addressed, this may lead to 
increased sensitivity and effectiveness of interventions for students that engage in NSSI behaviors.

\section{School-Based Professionals Serving Students: Foundations of Social Cognitive Theory}

Social learning theory, now termed social cognitive theory, refers to a change in cognition and behavior resulting from the observation of one or more models (Bandura, 1989; Ormrod, 2008). The process in turn allows the individual to develop a selfevaluating mechanism that assess whether or not they can accomplish a task or learn new material (Bandura, 1982, 1989).

There are several components of social-cognitive theory. A few of the components include triarchic reciprocal causality, human agency, and affect and selfefficacy (Bandura, 1989; Woolfolk, 2010). Triarchic reciprocal causality (the relationship between environment, behavior and person) describes the relationship between all of the variables and their impact on learning (Woolfolk, 2010). For example, motivation combined with self-efficacy and environmental factors (e.g., models, context in which learning occurs, etc.) may influence an individual's learning (Woolfolk, 2010). Self-regulation capabilities entail the use of personal agency and self-assurance. People who are skeptical of their ability to exercise adequate control over their actions tend to undermine their efforts in situations that tax capabilities. Mastery of problem situations further strengthens self-regulatory efficacy (Bandura, 1982).

Human agency and perceived self-efficacy. Human agency refers to the capacity to reach ones goals by directing motivation, learning skills and emotions 
(Bandura, 1982, 1989; Woolfolk, 2010). Agency involves the intentional development of goals, designing action plans to reach those goals and finally carrying out the necessary actions to meet those goals (Woolfolk, 2010). It is the inherent desire to navigate the environment that develops characteristics of agency (Bandura, 1982). Bandura (1989) defines emergent agency in relation to self-efficacy as the causal relationship between an individual's motivation, cognitions, affect as well as other personal factors that lead individuals to feel efficacious enough to complete tasks. Emergent agency directly relates to an individual's motivation to learn and approach new tasks with a high sense of selfefficacy.

The development of perceived self-efficacy is borne of these characteristics in which an individual directs their thinking and behavior, in a corresponding fashion, to accomplish a task (Bandura, 1989). The "Can I do it?" state of mind guides behaviors of individuals to attempt various tasks (Ormrod, 2009; Woolfolk, 2010). According to Bandura (1982), perceived self-efficacy is "concerned with judgments of how well one can execute courses of action required to deal with prospective situations" (p.122).

Perceived self-efficacy and task persistence. Perceived self-efficacy can impact an individual's ability to approach tasks and take risks. Individuals' appraisal of their capabilities influences their affect and cognitions while approaching tasks (Bandura, 1989; Ormrod, 2008). Self-doubt, in some instances, can influence learning and encumber the individual's ability to perform efficaciously (Bandura, 1982; 1989; Ormrod, 2008). For example, those individuals who feel efficacious may see little need in placing effort in preparing for tasks that may highlight their abilities (Bandura, 1982). 
Self-efficacy is also context specific and can affect an individual's preparatory and performance effort differently (Ormrod, 2008).

Yet, Bandura (1989) asserts that varying degrees of effort exerted in completing tasks are not a mishap. In fact, it may demonstrate an increased likelihood of the individual attempting challenging tasks in the future. How else will one move beyond the current state of learning and behaving if charting new territory is not met with tenacity? Self-efficacy also refers to the how much effort individuals will put forth and how long they will persist in the face of obstacles or aversive experiences (Bandura, 1982). If perceived self-efficacy is high, then motivation to engage in tasks and perform well at these tasks will be demonstrated (Bandura, 1982). This also means that individuals will persist in their tasks.

Related to this concept, individuals with prior experiences in performing various tasks may have higher self-efficacy when approaching future, yet similar tasks (Bandura, 1989). For example, if a general education teacher has successful experiences in providing interventions to an individual that engages in NSSI, they may feel more efficacious in providing those same interventions and services to the another individual engaging in similar behaviors. With this particular example, it should not be assumed that little effort will be exerted on the part of the individual when approaching the task at hand. The focus of the individual's efforts may be more on treating the individual rather than showcasing their abilities.

Affect and self-efficacy. Affect and self-efficacy represent an individual specific approach to learning. These concepts vary widely depending on individual differences as well as experiences. With respect to self-efficacy, Ormrod (2008) defines it 
as the belief that one is "capable of executing behaviors or performing task successfully" (p. 135). This relates directly to motivation and task persistence. For example, if an individual has low motivation and low task persistence because they have little confidence in their competence in completing a task, it is likely that they will have negative affectivity. In fact, because they are less than efficacious regarding the task, the person may not be interested in learning new materials and/or tasks.

Learning can positively or negatively impact an individual's affect. Consequently, these emotions feed directly into their ability to feel that they are competent enough to achieve their goals or master a task (Ormrod, 2004). If an individual feels optimistic and has a more positive affectivity towards various tasks in general, they are more likely to feel efficacious, or at least take a more positive perspective in regards to their ability to complete a task. Furthermore, because they feel confident and competent in their ability to master a task, they are more open to learn new information and illustrate high levels of task persistence (Ormrod, 2004).

Approaching new tasks and sources of self-efficacy. According to Woolfolk (2010), there are four sources of self-efficacy: mastery experiences, vicarious experiences, social persuasion, and physiological arousal. Mastery experiences are related to past successes and failures of the individual. A positive correlation exists between selfefficacy and an individual's ability, effort, choices, and their strategies to accomplish a task (Bandura 1982, Woolfolk, 2010). For example, school-based professionals are likely to be more responsive, learn new strategies, and feel efficacious to the needs of individuals who engage in NSSI behaviors, if their previous experiences with these individuals have been positive and helpful. Conversely, if school-based professionals feel 
as if they lack the knowledge necessary to intervene with individuals who engage in NSSI behaviors and thus failed to respond in an inefficacious manner, they may not exert much effort to learn, yet alone utilize new strategies. This was observed in previous studies with teachers (Heath, Toste, \& Beettam, 2006) as well as health and mental health professionals (Best, 2005) who had contact with individuals who engaged in NSSI behaviors.

Secondly, vicarious experiences, such as modeling, also influence an individual's feelings of self-efficacy. An individual's perceived self-efficacy can be enhanced by viewing other individuals who are deemed to be representations of themselves; be it status (student-student), profession (nurse-nurse), or other personal characteristics, succeed at the same task (Bandura, 1982; Ormrod, 2008). For example, school nurses who observe other school nurses successfully intervening with students engaging in NSSI behaviors may be more inclined to seek out training and information to boost their ability to work efficaciously with those same individuals (see Shapiro, 2008). Vicarious learning may occur when an individual participates in professional development or works on multidisciplinary teams with like individuals who possess knowledge or expertise in a certain area. This in turn influences their perceived self-efficacy.

Another component of self-efficacy is social persuasion. This includes encouragement, feedback, and guidance from a respected source (Woolfolk, 2010). For example, special education teachers may be more likely to serve on teams to assist students that engage in NSSI behaviors if a school or district administrator has provided training and encouragement to them. Social persuasion lends heavily to the idea of collective self-efficacy. Collective self-efficacy refers to the perception of an individual's 
ability, the ability of others and their combined abilities to accomplish goals (FernándezBallestros, Diez-Nicolás, Caprara, Barbaranelli \& Bandura, 2002; Ormrod, 2008). When individuals feel their abilities and efforts make a difference, their sense of self-efficacy increases. For example, when school counselors are working with individuals that engage in NSSI behaviors, they may feel more successful in approaching the task as a member of a multidisciplinary team (Best, 2005) In fact, Fernández-Ballestros et al. (2002), suggest that individuals are more effective when approaching and tackling a task together rather than individually. It is this shared agenda and cooperative framework that increases perceived self-efficacy in all individuals.

Finally, somatic arousal, be it positive or negative, can impact an individual's sense of self-efficacy (Bandura, 1982). It is the individual's emotional response as they approach a task that influences their responsiveness, cognitions, and behavior. For example, if an individual approaches a task with anxiety and fear, they are more likely to feel less efficacious than an individual who approaches the same task with excitement and confidence. This is related to the relationship between affect and self-efficacy explained in the previous section. The crux of the current study seeks to examine a number of variables that may impact school-based professionals self-efficacy when working with individuals that engage in NSSI behaviors.

\section{Pilot Study of Knowledge of NSSI Behaviors and Related Interventions}

Moore (2009) devised a pilot study to examine the knowledge base of schoolbased health and mental health professionals regarding self-injury. It was hypothesized that school psychologists, due to their intensive mental health training, would have significantly more general knowledge of self-injurious behaviors as well as be better able 
to recognize any myths or misconceptions surrounding self-injury than school counselors and school nurses.

The second hypothesis was that school psychologists would have significantly more knowledge and self-efficacy in developing effective interventions related to selfinjury than other professionals due to the nature of their training. School counselors and school nurses, respectively, were expected to follow regarding their knowledge on intervention for individuals engaging in self-injurious behaviors. Thirdly, it was hypothesized that school psychologists and school counselors would have significantly more capacity to provide interventions and services to individuals engaging in selfinjurious behaviors than school nurses. Finally, with respect to school placement, it was hypothesized that individuals who are placed at the secondary levels, in junior high and high schools, would have significantly more general knowledge, knowledge of interventions, as well as the capacity to carry out those interventions.

The study included 260 participants and was conducted in Fall 2006 (Moore, 2009). Out of the 260 participants, 230 provided responses sufficient for data analysis. Participation from school psychologists and school psychological examiners was solicited via the National Association of School Psychologist (NASP) listserv. Similar recruitment letters were distributed to members of the Missouri Association of School Counselors, National Association of Schools Counselors, and Missouri School Social Workers via their respective listservs.

Overall Results for Health and Mental Health Professionals. Results from the study indicated that there were significant differences in the knowledge base of school-based health and mental health professionals. As predicted, school psychologists 
had statistically and significantly $(\mathrm{p}<.05)$ greater general knowledge score regarding selfinjury than school nurses. While school counselors' scores were lower than school psychologists and higher than school nurses, these differences were not statistically significant, and thus these hypotheses were not supported.

Analysis of item responses revealed that the general knowledge portion of the survey showed that most school psychologists and school counselors have accurate knowledge of self-injury with respect to its association with psychological disorders (e.g., depression, eating disorders, etc.) and common features (e.g., injurious behaviors occurring in secrecy, self-injurious behaviors are a coping mechanism). However, the knowledge of etiology and underlying mental health issues presented an area of uncertainty for these professionals. These findings may suggest that school-based professionals may have encountered students who engage in self-injurious behaviors, but have little to no knowledge of how self-injury develops in individuals.

Next, I hypothesized that school psychologists would have significantly more knowledge of interventions related to self-injury than school counselors and school nurses. Interestingly, the results indicated that school nurses had statistically and significantly $(\mathrm{p}<.05)$ more perceived knowledge of interventions than school counselors. There was an observed trend that their knowledge of interventions score was higher than all other professionals, with school psychologists following with the next highest score, and then school counselors.

The third hypothesis that school psychologists would have significantly more capacity to carry out interventions was not supported by study results. There were no significant differences, by the role of the health or mental health professional, in 
perceived ability to provide interventions or suggestions with respect to self-injury. It is surprising that health and mental health professionals did not perceive that they had the latitude or resources to carry out interventions. Most of these professionals were often part of multidisciplinary teams in schools and typically had access to other school-based professionals that serve as resources.

Though not statistically significant, school nurses had a higher score indicating more perceived capacity to carry out interventions followed by school psychologists then school counselors. This may be supported by the fact that school nurses are trained in crises interventions and play a very important role in carrying out safety plans (Shapiro, 2008). This finding may also tap into a self-efficacy issue that warrants further exploration.

Finally, results indicate that professionals serving children in high schools had a significantly greater level of knowledge of self-injurious behaviors over those professionals serving students across multiple schools. Multiple schools in the study could mean a number of schools from elementary to high school for which professionals are responsible. This partially supports the hypothesis of the pilot research. The finding suggests that school placement plays an integral role in the exposure of school-based professionals to NSSI behaviors more than likely due to the age of onset for individuals.

Survey Reliability. Reliability analysis for the original distinct sections of the measure yielded a Cronbach's Alpha ( $\left.{ }^{\alpha}\right)$ of .550 for section 2, .431 for section 3, and .356 for section 4 . Improvements made to the measure used in the current study include rewording of confusing and double-barreled questions. Additionally, new items were created for the general knowledge section. These items reflect updated prevalence rates, 
related interventions, and associated psychiatric disorders with NSSI behaviors. Lastly, based on recommendations and comments from survey participants, items were deleted or reworded and a self-efficacy section was added to the survey. This new section was added to determine to how efficacious school-based personnel feel when working with individuals who engage in NSSI behaviors. Additionally, professionals' general knowledge of NSSI behaviors was examined to determine how it impacts their selfefficacy.

Limitations of the Pilot Study. The data analysis in the pilot study was problematic in a number of ways on which the current study seeks to improve. One flaw of the pilot study was the lack of general mean comparisons among a variety of schoolbased professionals. Variables general knowledge of NSSI, knowledge of related interventions, and the capacity to implement those interventions were calculated for each school-based professional. Due to the relatively small sample size of school social workers $(n=5)$, this group was dropped and consequently not included in the analyses. Collapsing the group with other mental health professionals may have offered a complete picture of all mental health professionals on the aforementioned three variables. Additionally, teachers and administrators were not included in the pilot sample. Exploring the relationships among the variables by role might have been more meaningful than across group comparisons alone. The current study will examine all mean difference across measure constructs as it relates to role of the professional and their direct experience engaging in NSSI behaviors.

School-based professionals' direct experience in working with students that engage in NSSI behaviors was not examined in the pilot study. This variable may have 
acted as a mediator and/or moderator in explaining mean differences across professions with respect their general knowledge of NSSI behaviors and related interventions. Additionally, it may have mediated differences in self-efficacy in developing interventions, delivering services, and working with individuals that engage in NSSI behaviors across professions. The current study seeks to examine this important variable.

\section{Rationale for Current Study}

As a result of the growing number of children and adolescents diagnosed with mental health problems, schools are attempting to address the needs of students. Additionally, schools are encouraged to engage in practices that effectively address mental health wellness for all students through evidence-based prevention and early intervention practices (Reinke et al., 2011). Researchers and practitioners alike recognize how mental health problems could adversely affect learning (Lieberman, Toste, \& Heath, 2009; Ormord, 2009). The thrust of these practices is categorized as prevention and early intervention practices. Consequently, schools have developed crisis intervention teams, screening methods as well as school- and classroom-based interventions to address mental health problems and their associated features (Lieberman, Toste, \& Heath, 2009). Of these features, NSSI is a common thread among several mental health disorders such as depression, anxiety, and obsessive-compulsive disorder.

While assessment and intervention tools for self-injurious behaviors are available, the question remains whether or not school-based professionals have the knowledge, skills, and self-efficacy to respond to concerns of this nature. School-based professionals are on the frontlines of identifying and treating individuals with mental health issues 
(Heath, Toste, Sornberger, \& Wagner, 2011). Possessing knowledge of and having direct experience with mental health disorders and features such as non-suicidal self-injury could greatly influence the self-efficacy of helping professionals (Lieberman, Toste, \& Heath, 2009). For example, school-based professionals could target the distorted thinking of individuals who engage in NSSI behaviors in order to address feelings of inadequacy, loss, rejection, and fear (Alderman, 1997; Glassman, Weierich, Hooley, Deliberto \& Nock, 2007; Lieberman, Toste, \& Heath, 2009; Lloyd-Richardson, Perrine, Dierker, \& Kelley, 2007). With these critical elements in mind, it is important to clearly define and understand NSSI with respect to various clinical and general populations, particularly school-based populations. Additionally, discussing intervention and prevention strategies used in schools to address this growing concerning among adolescents is paramount (Lieberman, Toste, \& Heath, 2009).

Further, school-based professionals were selected to participate in the study for their vital roles in serving and educating children in the schools. Because of the likelihood that school psychologists, school counselors, and school social workers will provide information to school staff and students regarding NSSI and related behaviors, the researcher seeks to explore their general knowledge. Additionally, those who may receive information from these school-based health and mental health professionals, such as teachers and school/district administrators will be asked to share their current knowledge and level of self-efficacy in providing interventions and recommendations if confronted with this issue in their school. Similarly, school nurses are involved in treating students with medical or health-related concerns. Individuals who engage in selfinjurious behaviors are at risk for sustaining moderate to severe physical damage 
(Prinstein et al., 2008), and thus school nurses may play a significant role in addressing such concerns. Collaborative efforts to address NSSI behaviors and other mental health concerns are critical. The involvement of multiple professionals across disciplines and families of students with identified mental health concerns may prove to be an effective partnership. Nonetheless, because the aforementioned professionals are on the frontlines of intervening with students who may engage in NSSI behaviors, they are included in this timely and relevant study to ensure the safety and healthy socioemotional development of children.

In sum, this study digests the limitations of the pilot (Moore, 2009) and offers a number of strategies for improving on these limitations. First, the researcher will collapse across mental health, health, and educator roles to include all school-based professionals that participated in the study. That is, professional groups with few respondents will be added to closely related professionals groups with more respondents. This will insure that every response is accounted for and that groups will have more robust representation in analyses involving group comparisons.

Secondly, previous study findings suggest that most school-based professionals, overall, do not hold a high level of knowledge nor confidence in working with students engaging in NSSI behaviors (Best, 2005; Heath, Toste, Sornberger, \& Wagner, 2011; Moore, 2009). It is the intention of this study to further explore related variables that may explain this finding. The researcher has identified two mediating variables that were not measured in the pilot study. These variables are (a) school-based professionals' direct experience in working with students that engage in NSSI behaviors and (b) experience with various mental health disorders. These variables will offer a more complete picture 
of the relationship between role and knowledge of NSSI behaviors as well as role and perceived self-efficacy than just examining the relationships alone.

Third, the researcher will address the poor reliability of the pilot measure by increasing the number of items in each section; utilizing an extensive, current literature review in the development of measure items; and sampling the study with the intended population for input on readability and clarity in the measure items. To determine how well the measure assesses the true knowledge and perceived self-efficacy of school-based professionals, an analysis of internal consistency will be utilized.

Internal consistency refers to how well the observed score and true score are estimated to reduce the amount of unexplained or measurement error. Reliable instruments are critical to the use of path analytic methods like those proposed in this study (Olobatuyi, 2006; Tabachnik \& Fidell, 2012). There are several methods of assessing reliability of measures; however, due to the procedures and measures applied in this study, internal consistency is ideal. Internal consistency will be measured using Cronbach's alpha and composite reliability for each quantitative section of the instrument.

Finally, one of the main goals of this study is to investigate the level of selfefficacy of school-based professionals working with students who engage in NSSI behaviors. This will be accomplished by assessing the extent to which school-based professionals' have direct experience in working with students who engage in NSSI behaviors and their experience with mental health disorders. The proposed model addresses the limited scope of analysis presented in the pilot and is supported with social cognitive theory (SCT) as described above. Inquiry into these areas is extremely timely 
and relevant as all school-based professionals share a role in creating a safe school environment for students (Lieberman, Toste, \& Heath, 2009; Reinke et al., 2011).

Mental-health professionals such as school counselors and school psychologists are trained to identify and treat students with various mental health disorders (Best, 2005). However, the specific training of school psychological examiners, teachers, school/district administrators, and school nurses in working with individuals who engage in NSSI behaviors remains unclear. According to Social Cognitive Theory (SCT), perceived self-efficacy may play an integral role in professionals learning new information and applying this information in working with individuals that engage in NSSI behaviors. Further, SCT would predict that the higher the level of prior knowledge and direct experiences in a particular tasks, the higher the perceived self-efficacy and future task persistence.

Recent literature has alluded to the pre-service and in-service training of school nurses with respect to crises intervention and mental health disorders as they work in the physical treatment of these individuals (Best, 2005; Shapiro, 2008). There has been a question as to the knowledge and usage of evidence-based interventions for mental health disorders in general (Kratochwill \& Shernoff, 2004). With the information contained herein, the proposed study identifies variables associated with preparedness in working with students that engage in non-suicidal self-injurious behaviors. This will ultimately inform training and current practices as researchers and professionals develop tools to assist professionals. 


\section{Research Questions and Study Hypotheses}

Examining relationships between predictor and outcome variables alone provides only a portion of the picture. Related to this study, it is important to understand how other variables may explain variance among professionals based on their role in schools. To provide a more accurate explanation that accounts for the variance between these complex relationships, mediators are proposed and tested. A mediator is an intermediate variable that specifies further additional factors that may help to explain the causal relationship between predictor and outcome variables.

This study examines outcome variables, professional's general knowledge and perceived self-efficacy, which are predicted from the school-based professional's role and mediated by professional's direct experience with NSSI behaviors and experience in working with students who have mental health disorders. Experience working with students with various mental health disorders may sensitize school-based professional to recognize and seek additional information to assist students engaging in NSSI behaviors. This is an uncharted area of research and is the intent of the current study to explore this relationship has it relates to the role of the school-based professionals.

Research Question \#1: Are there certain school-based professionals that possess greater knowledge of NSSI behaviors and perceived self-efficacy due to their direct experiences working with individuals engaging in NSSI behaviors and knowledge of mental health disorders?

Secondary Research Question \#1A: Overall, is role of school based professional significantly associated with knowledge of mental health 
disorders, knowledge of NSSI behaviors, and experience with perceived selfefficacy working with students engaging in NSSI behaviors?

Secondary Research Question \#1B: If there is a significant multivariate effect, is there a significant univariate effect of school-based professional roles on the dependent variables?

Secondary Research Question \#1C: Given a significant univariate effect, which roles are significantly associated with each dependent variable?

Hypothesis \#1: Professionals with more direct experience with individuals engaging in NSSI and familiarity with various mental health disorders, will have higher general knowledge of NSSI behaviors and perceived self-efficacy than individuals with less direct experience based upon their specific role in school.

Research Question \#2: Does direct experience with individuals engaging in NSSI behaviors and familiarity of various mental health disorders significantly mediate the relationship between role of school-based professional and their perceived self-efficacy?

Secondary Research Question \#2A: Is the direct effect significantly greater than the indirect effect between role of school-based professional and their perceived self-efficacy as mediated by direct experience with NSSI behaviors? 
Secondary Research Question \#2B: Is the direct effect significantly greater than the indirect effect between role of school-based professional and their perceived self-efficacy as mediated by familiarity with various mental health disorders?

Hypothesis \#2: Professional's direct experiences with NSSI behaviors and familiarity with various mental health disorders will significantly predict their perceived selfefficacy based upon their role in the school.

Research Question \#3: Does direct experience with individuals engaging in NSSI behaviors and familiarity of various mental health disorders significantly mediate the relationship between role of school-based professional and their general knowledge of NSSI behaviors?

Secondary Research Question \#3A: Is the direct effect significantly greater than the indirect effect between role of school-based professional and general knowledge of NSSI behaviors as mediated by direct experience with NSSI behaviors?

Secondary Research Question \#3B: Is the direct effect significantly greater than the indirect effect between role of school-based professional and general knowledge of NSSI behaviors as mediated by familiarity with various mental health disorders? 
Hypothesis \#3: Professional's direct experience with students engaging in NSSI

behaviors and familiarity with various mental health disorders will significantly predict their general knowledge of NSSI behaviors based upon their role in the school. 


\section{CHAPTER THREE \\ METHODS}

\section{Participants}

Participants were recruited from general education teachers, special education teachers, school psychologists, school psychological examiners, school counselors, school nurses, school social workers, school and district administrators and any other self-identified professionals working with children and adolescents in schools.

Participants were also recruited from the following organizations: Missouri Association of School Nurses, American School Counselors Association, and School Social Work Association of America. The following school districts agreed to participate in the study: Mexico School District 59 (Mexico, MO), Boonville R-1 School District (Boonville, MO), Southern Boone School District (Ashland, MO), Fulton 58 School District (Fulton, MO), Moberly Public School District (Moberly, MO), North Kansas City School District (Kansas City, MO), Hazelwood School District (St. Louis, MO), and Harrisburg School District (Harrisburg, MO).

Final demographic information for all survey participants are reported in Tables 1, 2, 3, and 4. For the purposes of analysis, School Counselors, School Psychological Examiners, and School Psychologists were combined to form the Mental Health/Counseling Associate group. These groups were combined because of their similar backgrounds in mental health training and service delivery and low representation in the current study. Information with respect to race, ethnicity and gender of professionals were not obtained, as it was not the focus of this study. 
Table 1

Observed Number of Participants by School-Based Professional's Role (Total N = 333)

\begin{tabular}{|c|c|c|c|c|c|c|c|}
\hline $\begin{array}{l}\text { Number of } \\
\text { Professionals }\end{array}$ & $\begin{array}{l}\text { Support } \\
\text { Staff }\end{array}$ & $\begin{array}{l}\text { School } \\
\text { Social } \\
\text { Worker }\end{array}$ & $\begin{array}{l}\text { School } \\
\text { Nurse }\end{array}$ & $\begin{array}{l}\text { Regular } \\
\text { Ed } \\
\text { Teacher }\end{array}$ & $\begin{array}{l}\text { Special } \\
\text { Ed } \\
\text { Teacher }\end{array}$ & $\begin{array}{c}\text { Mental } \\
\text { Health/ } \\
\text { Counseling } \\
\text { Associate }\end{array}$ & $\begin{array}{l}\text { School } \\
\text { or } \\
\text { District } \\
\text { Admin }\end{array}$ \\
\hline & 27 & 72 & 30 & 131 & 36 & 22 & 15 \\
\hline
\end{tabular}

Table 2

Number of Identified School Placements by Grade Level (Total N=333)

\begin{tabular}{lcccccc}
\hline $\begin{array}{l}\text { Number of } \\
\text { Professionals }\end{array}$ & $\begin{array}{c}\text { Preschool/Early } \\
\text { Childhood }\end{array}$ & Elementary & $\begin{array}{c}\text { Middle } \\
\text { School }\end{array}$ & $\begin{array}{c}\text { Junior } \\
\text { High } \\
\text { School }\end{array}$ & $\begin{array}{c}\text { High } \\
\text { School }\end{array}$ & $\begin{array}{c}\text { Other } \\
\text { Combined }\end{array}$ \\
& 12 & 128 & 82 & 5 & 94 & 12 \\
\hline
\end{tabular}

Table 3

Education Level of School-Based Professionals (Total $\mathrm{N}=332,1$ missing)

\begin{tabular}{lcccccc}
\hline $\begin{array}{l}\text { High } \\
\text { Number of }\end{array}$ & $\begin{array}{c}\text { School } \\
\text { Diplo } \\
\text { ma }\end{array}$ & Associates & Bachelors & Masters & $\begin{array}{c}\text { Education } \\
\text { Specialist }\end{array}$ & Doctorate \\
& 8 & 13 & 74 & 203 & 24 & 10 \\
\hline
\end{tabular}

Table 4

School-Based Professionals by U.S. Region (Total N = 333)

\begin{tabular}{lccccc}
\hline Number of & North & South & West & East & Midwest \\
Professionals & 9 & 18 & 13 & 10 & 283 \\
& & & & & \\
\hline
\end{tabular}




\section{Instrumentation}

Non-Suicidal Self-Injury Survey for School-Based Professionals. In order to capture the knowledge base of self-injury of school-based professionals, the Non-Suicidal Self-Injury Survey for School-Based Professionals measure was created. This survey assesses both factual information and quality of knowledge regarding selfinjurious behaviors based upon current literature and clinical studies. It also taps into constructs related to mental health disorders and perceived self-efficacy. The various sections are described briefly below and a copy of the survey is provided in Appendix F.

Demographic Section. The survey is divided into five sections. The first section of the measure requests demographic information from each survey participant. This information includes indicating the region of the U.S. in which they work (North, South, East, West, Midwest); their professional role in schools (e.g., educator, school nurse, school psychologist, school social worker, school counselor, and school psychological examiner, school administrator, and district administrator); assigned school level, and respective school district in which they work. The intent of the current study is to explore the knowledge of school-based professionals as it relates to their self-identified role in the school and to request information from a variety of professionals. The demographic section also requests the participant indicate the number of direct experiences working with students engaging in NSSI behaviors and experience working with individuals with various mental health disorders.

General Knowledge of NSSI Behaviors. The general knowledge section consists of 27 items utilizing a 6-point Likert-scale $(5=$ Strongly Agree to $1=$ Strongly Disagree, and $0=$ Do Not Know) for general knowledge questions. This section requests information about the participants' general knowledge about self-injury and those who 
engage in self-injurious behaviors. More specifically, items are presented as statements based on current, evidence-based findings presented throughout scholarly articles, clinical trials, as well as published individual clinical cases. Other statements in this section reflect common myths and misconceptions associated with NSSI and those who engage in self-injurious behaviors. Items that require a negative response are 5, 6, 8, 9, $10,11,12,13,17,18,21,23,26$, and 27 are reverse coded. All other questions require that the participant respond by agreeing with the statement. A general knowledge score was calculated by summing all responses for participants on the knowledge scale. This will serve as a dependent variable and used to compare mean differences among professionals. Higher general knowledge sum scores imply higher accuracy in knowledge of NSSI behaviors. Due to errors in scale development, this section was recoded to reflect right ( 2 points), wrong ( 0 points) and do not know or neutral ( 1 point) responses to formulate the general knowledge score.

Knowledge of Interventions for NSSI Behaviors. $\quad$ The third section includes 9items and examines participants' current knowledge of recommendations, services, and/or interventions for students and families surrounding non-suicidal self-injury. This section utilizes a 5-point Likert scale ( $5=$ Strongly Agree to $1=$ Strongly Disagree $)$. Knowledge of Intervention scores are calculated by summing the corresponding values to responses for all section questions. Though included in pilot study analyses, it was not included in the current study. The current study's focus is solely on general knowledge and perceived self-efficacy as mediating by direct experiences and familiarity with various mental health disorders. This offers another perspective as it builds upon the current body of NSSI literature. 
Perceived Self-Efficacy. The fourth section of the survey consists of 8-items that request information from participants about their perceived self-efficacy in providing services, suggestions, recommendations and interventions to students and families related to NSSI. This section also utilizes a 5 -point Likert scale $(5=$ Strongly Agree to $1=$ Strongly Disagree). Here, a higher score reflects higher perceived self-efficacy. Perceived self-efficacy scores were obtained by summing school-based professionals' responses across all questions in the section.

Topical Questions and Comments. The fifth and final section is an open-ended question designed to gauge what school-based professionals believe about NSSI in general and their relative knowledge and training opportunities. This section also serves as an opportunity for professionals to comment about the survey and their experiences around NSSI. Common themes will be extracted to reflect school-based professionals' thoughts and opinions.

Study Mediating Variables. Direct experience with NSSI behaviors and familiarity with various mental health disorders serve as mediating variables in the path analysis presented in the next chapter. Questions presented in the demographic section of the survey served as measures for these variables. General knowledge of NSSI behaviors was measured by participants indicating their level of direct experience using the following scale: $1=$ None, $2=$ One or two experiences, $3=$ Some experiences, $4=$ Regular experiences, and $5=$ Very regular experiences. Familiarity with various mental health disorders was measured by participants indicating the number and type of mental health disorders they possess knowledge and working experience of while working with students. The following scale was used: Depression, Anxiety, Obsessive Compulsive 
Disorder, Oppositional Defiant Disorder, Conduct Disorder, Tourette's Syndrome, NonSuicidal Self-Injury, Eating Disorders, Others.

\section{Procedures}

The University of Missouri's Institutional Review Board (IRB) approved the current study prior to dissemination. Individual district IRB approval was obtained for the Hazelwood School District and North Kansas City School District prior to dissemination of the survey to professionals within the district. Other districts participated with consent form the superintendent. Districts were contacted via phone to schedule a personal meeting with the superintendent and the researcher. Upon meeting with the superintendent, the study was explained in detailed and a free professional development around the NSSI was offered in exchange for the district's participation. This face-to-face method proved to be $100 \%$ effective in enrolling districts in the study.

Initial drafts of the survey were closely reviewed for grammatical errors, sentence structure, and clarity with a professional from MU Student Success Writing Center. The 2-hour process yielded a measure that reworded ambiguous items and deleted redundant items. The measure was then administered to a small representative sample of the intended population. The group consisted of 3 teachers, 1 school psychological examiner, 1 school counselor, and 1 school administrator. This method also tested the measure for face validity. Suggestions regarding the wording or presentation of items were obtained and incorporated into the development process when applicable. Final adjustments of the survey, based on feedback from both groups of reviewers, were applied. 
Participants were recruited on a voluntary basis and offered a contingent incentive in which they may choose to enter a raffle for 1 of $20 \$ 20$ Mastercard/Visa gift cards. Out of the 21 schools asked to participate in the study, eight agreed. The survey was placed online using an online survey program, SurveyMonkey. SurveyMonkey allows for webbased administration of survey questionnaires and storage of survey responses in a secure, password protected database. Using capabilities of the online survey program, anonymity of participant responses were protected. Response rates for online surveys range from 20-30\% (Kaplowitz, Hadlock \& Levine, 2004). The researcher observed typical response rates for school administrators and educators. The observed response rates for organizations were minute in comparison to district participation. Reponses were organized by the online survey program and exported to IBM SPSS 21.0.

The link to the study's survey was included in a short introductory paragraph (Appendix C) emailed to district administrators who agreed to participate in the study. The call for participation petitioned potential subjects to complete an online survey through Surveymonkey (see Appendix F). Full recruitment and consent letters were a part of the survey created online. The recruitment/consent letter (Appendix A) highlighted the relevance of non-suicidal self-injurious behaviors to their practice within the schools. Introductory paragraphs and solicitation scripts (Appendix D) were disseminated through district's school-wide listservs or mass emailing systems. 


\section{CHAPTER FOUR}

DATA ANALYSIS

\section{Research Design}

The purpose of this study was to assess school-based health professionals' knowledge of NSSI behaviors and their perceived self-efficacy when working with individuals that engage in NSSI behaviors. The study also examines mediating variables, direct experience with NSSI behaviors and familiarity with mental health disorders, to determine how they influence school-based professional's perceived self-efficacy and general knowledge of NSSI behaviors. The current study has an intact comparison and survey research design. Intact research designs use groups that are currently in place based upon self-identified characteristics of study participants rather than assigning individuals to researcher constructed groups. For example, for this study, participants are selected based upon their profession as a school nurse, school counselor, general education teacher and so forth, and were not randomly assigned to those groups.

To assess school-based professionals' general knowledge of NSSI and perceived self-efficacy in working with students that engage in NSSI a survey instrument was developed. Reliability statistics were obtained for the instrument. Data analysis also involved calculation and comparison of mean differences across various school-based professionals with respect to general knowledge of NSSI, interventions related to NSSI, and professionals' perceived self-efficacy. Bivariate correlations are examined and displayed in a correlation matrix. This matrix provided the first step leading to the determination of path coefficients presented in the path analysis model. Lastly, the study 
examined mediating effects of direct experiences with NSSI and experiences with mental health disorders on professionals' general knowledge and perceived self-efficacy.

\section{Multivariate Analysis of Variance (MANOVA) Assumptions}

A Multivariate Analysis of Variance (MANOVA) was used to analyze the effects of mean differences in school-based professional's role on general knowledge of NSSI behaviors and perceived self-efficacy. Prior to conducting the formal multivariate analysis of variance procedures, the data were examined to ensure that the MANOVA assumptions would support the use of this procedure for data analysis. The three assumptions that were examined are: independence of observations, normal distribution of dependent variables, and homogeneity of variance-covariance matrices.

Independence of Observations. The independence assumption will be examined by determining if study participants worked independently on the survey instrument.

Normal Distribution of Dependent Variables. Inspection of the study data through histograms, skewness (maximum values exceed \pm 1 ) and kurtosis (maximum values exceed \pm 3 ) statistics, as well as Shapiro-Wilks statistics, were used to determine if the data is normally distributed. Positively skewed distributions are tall, wide, and shifted to the left with smaller tails to the right, indicating means fall to the left rather than the center of the distribution. Positive kurtosis distributions are observed to be extremely peaked and steep with short, thick tails indicating that more scores are found in the tails, which may underestimate the variance of a particular variable (Keppel \& Wickens, 2004; Tabachnik\& Fidell, 2007). 
Homogeneity of Covariances and Variances. Finally, to support the assumption of homogeneity of covariances and variances, Box's and Levene's test was used, respectively. Box's test will be used to test whether population covariance matrices for the dependent variables are equal across groups. Levene's test will be used to test whether variances of univariate tests are equal across groups.

\section{Linear Regression and Path Analysis Assumptions}

Prior to conducting a path analysis, the data were examined to ensure various assumptions were met. The assumptions are discussed as follows:

Normal Distribution of Dependent Variables. Inspection of the study data through histograms, skewness (i.e., significant skewness is inferred if maximum values exceed \pm 1 ) and kurtosis (i.e., significant kurtosis is inferred if maximum values exceed \pm 3 ) statistics, as well as Shapiro-Wilks statistics, was used to determine if the data is normally distributed. Positively skewed distributions are tall, wide, and shifted to the left with smaller tails to the right indicating means fall to the left rather than the center of the distribution. Positive kurtosis distributions are observed to be extremely peaked and steep with short, thick tails indicating that more scores are found in the tails, which may underestimate the variance of a particular variable (Keppel \& Wickens, 2004; Tabachnik \& Fidell, 2012). Violations of this assumption can be addressed through several transformation methods such as square root and natural log of observations methods (Olobatuyi, 2006).

Linearity. The use of path analysis assumes that variables are linearly related. This means that as values of variable $\mathrm{X}$ increases there is a corresponding increased incremental change in the units of variable $\mathrm{Y}$ and vice versa. This is true while holding 
other independent variables constant. This is necessary to explain variation in outcome variables in relation to the variance in predictor variables. Additionally, linearity increases prediction accuracy and decreases prediction error (Olobatuyi, 2006; Tabachnick \& Fidell, 2012). Plotting unstandardized residuals against independent and predicted variables tests linearity. Loess lines are used to determine fit of plots. Violations of this assumption will lead to biased regression coefficients and standard errors resulting in estimates that are not representative of true population parameters.

Interval Level of Variables. All variables must be on interval or ratio scales of measurement to conduct a path analysis (Olobatuyi, 2006). This is necessary to ensure accurate predictions in the model. This is also especially important in complex models such as those proposed in the current study (Olobatuyi, 2006). All measured variables in the data set are on an interval or ratio level of measurement. Violations of this assumption would not allow for proper data analysis and will ultimately lead to inaccurate and unreliable findings.

Lack of Measurement Error. In a path analysis it is essential to have reliable independent variables that are selected a priori and free from measurement error to insure accurate parameter estimates and variances. In other words, the measure used to obtain the variables used in the analysis should be reliable so that model variables are equally reliable. This is achieved by utilizing various internal consistency reliability estimates such as the Cronbach's Alpha Coefficient, Kuder-Richardson or the test-retest method (Olobatuyi, 2006; Tabachnick \& Fidell, 2012). Violations of this assumption would result in errors in path coefficient estimates thus impacting accurate interpretations of the 
coefficient. Responses on instruments have acceptable reliability with coefficient estimates of .70 or better (George \& Mallery, 2003).

Homoskedasticity. Error terms in a path analysis should remain constant as they are distributed across independent variables (Olobatuyi, 2006). This assumption determines if error variance is constant around the regression line at various values of independent variables. When violated, the variances at each value of independent variables are not all equal. Plotting saved residuals against each predictor variable and values tests this assumption. Violations of this assumption result in inaccurate standard errors and ultimately inaccurate significance tests. When this assumption is violated, Weighted Least Squares Regression can be used to assign observations with high variance a low weight value and those with low variance will be assigned a high weight value.

Lack of Multicollinearity. Multicollinearity addresses the issue of correlation between independent variables. Violations of this assumption appear when independent variables are highly correlated, thus demonstrating an approximate linear relationship or exhibiting perfect multicollinearity (i.e., a correlation or multiple correlation of .90 or greater). In either scenario, regression coefficients may be found to lack statistical significance due to large standard errors (Olobatuyi, 2006; Tabachnick \& Fidell, 2012). When this assumption is violated, collapsing independent variables into one predictor variable, if a common theoretical concept is present, may address this issue (Olobatuyi, 2006).

Model Specification. Accuracy in model specification ensures reliable estimates of model parameters. It is vital to path analysis that the model is constructed 
based on sound theory only including the most relevant variables in the model. This assumption also entails entering variables into the model correctly to obtain accurate estimates. Lack of Fit Test and sound theory can be used to test model specification (Olobatuyi, 2006). Violations of this assumption jeopardize statistical significance test values (tests are too sensitive) and the models ability to correctly estimate parameters.

Model Identification. Path models that are identified have the same number of observed correlations as there are path coefficients or implied moments. This is important to obtain accurate estimates of path coefficients or unknown parameters from the structural model (Olobatuyi, 2006). This assumption can be tested to determine if the model is just identified, over-identified, or under-identified by using structural equations for each covariance and variance as a function of the path coefficients (Olobatuyi, 2006; Tabachnick \& Fidell, 2012). Models that are just-identified have an equal number of equations to determine path coefficients as there are covariances between variables. Conversely, models that are under-identified have more covariances between variables in the model than there are equations used to determine path coefficients. Over-identified models have more equations for path coefficients than there are variable covariances. Violations of this assumption depend on the type of identification error. Models that are under-identified result in inaccurate estimates of path coefficients. This can be addressed through the use Ordinary Least Square (OLS) method, but estimates will be inconsistent (Olobatuyi, 2006). Models that are over-identified results in undefined parameter estimates. One last note, the number of equations used to calculate path coefficients in the current model does not necessarily equal the total number of unique (i.e., not counting 
diagonals) bivariate correlations. Identified model with independent and dependent variables parsed out by their associated path components is shown in Table 5 . 
Table 5

Variables Used to Calculate Path Analysis Coefficients

\begin{tabular}{lll}
\hline Model & Independent Variables & Dependent Variables \\
\hline Non-Suicidal Self Injury & $\begin{array}{l}\text { Direct Experience with } \\
\text { NSSI Behaviors* }\end{array}$ & Perceived Self-Efficacy** \\
& Professional's Role & \\
Familiarity with Mental & \\
Health Disorders* & \\
& Professional's Role & NSSI Direct Experiences* \\
& Professional's Role & Familiarity with Mental \\
& Health Disorders* \\
& $\begin{array}{l}\text { Direct Experiences with } \\
\text { NSSI Behaviors* }\end{array}$ & General Knowledge \\
Familiarity with Mental & \\
Health Disorders* & \\
Professional's Role & \\
&
\end{tabular}

*Variable serves as mediator in models. ${ }^{* *}$ Denotes outcome variable in models.

\section{Transformation Procedure to Satisfy Normality Assumption}

After conducting the normality tests listed above and the data appears to be seriously non-normally distributed, transformation methods will be employed.

Transformation for group data is recommended should the dependent variables prove to violate skewness and kurtosis maximums (Tabachnick \& Fidell, 2007). Transformation methods are used to make the mean difference the median difference which is a better "measure of central tendency" when distributions are skewed (Tabachnick \& Fidell, 
2007). In addition, transformation methods are used to make the mean independent of the variance so data is normally distributed (Bland \& Altman, 1996).

Data can be transformed in a number of ways depending on the shape of the distribution and severity of non-normality. For data that is moderately non-normal, the square root transformation is recommended. For significantly non-normal data the logarithm method is recommended while the inverse is suggested for severely nonnormal data (Tabachnick \& Fidell, 2007). Using the SPSS 21.0 statistical program, variable transformation methods will be utilized by entering the mathematical shorthand for square root (sqrt) or inverse and adding each item within the dependent variable to the transformation equation. Normality statistics will be obtained again to determine the effectiveness of the transformation method. It is recommended; however, to attempt various methods to gain the best results for a normally distributed variable (Tabachnik \& Fidell, 2007).

\section{Missing Data and Unequal Cell Sizes Analysis}

Several missing data treatment methods were employed. Upon inspection of the missing data, the researcher determined that that the missing data was completely at random. Additionally, missing data can result in unequal cell sizes, thus affecting data analysis and generalizability (Tabachnik \& Fidell, 2007). Cell sizes as well as research

priorities and methods will be examined prior to conducting the MANOVA to determine whether Type I, II, III, or IV Sums of Squares should be used. Type I method is used when cell sizes are unequal, the study is nonexperimental, and the researcher's seeks the freedom "to set up the sequence of adjustment of covariates, main effects, and 
interactions" (p. 218, Tabachnik \& Fidell, 2007). It is recommended if unequal cell sizes are observed and the study is survey or nonexperimental, Type II Sums of Squares should be utilized. Type II Sums of Squares involves "adjusting for each effect except terms that 'contain' the effect being tested” (p.163, Langsrud, 2003). Type III and Type IV Sums of Squares, the most widely used and conservative, assume equal weighted cell sizes and are typically employed in experimental studies (see Tabachnik \& Fidell, 2007).

\section{A Priori and Post Hoc Power Analysis}

Power is the probability of detecting effects that have statistical significance in the data analysis (Tabachnik \& Fidell, 2007). An a priori and post hoc power analysis using IBM SPSS Sample Power 3.0 and general statistic program 21.0, respectively, will be conducted to determine if "low power has affected the internal validity of the findings." (Onwuegbuzie \& Leech, 2004). A priori power analysis helps determine the sample size needed to obtain significant findings (e.g., effect sizes); while post hoc power analysis guides future research by analyzing actual sample sizes that obtained significant effect sizes. The criteria for determining acceptable power for the study will be .80 or greater recommended by Jacob Cohen (see Onwuegbuzie \& Leech, 2004; Tabachnik \& Fidell, 2007). According to a priori power analysis results conducted by the statistical program IBM Sample Power 3.0, the study has sufficient power to achieve statistical significance in the findings $(.95, \mathrm{p}<.01)$. 


\section{Proposed Bivariate Relationships and Analysis}

Proposed directions of point-biserial correlations of study variables are presented in Table 6. Point-Biserial correlations with superscripts are school-based professional role dependent. For example, a negative point-biserial correlation will be observed for teachers (e.g., special education teachers) who have little direct experience with NSSI behaviors. Conversely, a positive point-biserial correlation will be observed with school nurses and their direct experience with NSSI behaviors. Lastly, this matrix will be used to calculate path coefficients.

Prior to conducting the path analysis, point-biserial correlations among all variables in the model will be examined (Olobatuyi, 2006; Tabachnik \& Fidell, 2007). Partial correlations will be examined to determine if point-biserial correlations are a true reflection of all underlying processes impacting the correlation. Partial correlations allow for "pairwise correlations that are adjusted for effects of all other variables" (Tabachnik \& Fidell, 2007). 
Table 6.

Predicted Directions of Point-Biserial Correlations for Model

\begin{tabular}{|c|c|c|c|c|c|}
\hline Variable & $\begin{array}{c}\text { Role of } \\
\text { Professional }\end{array}$ & $\begin{array}{c}\text { Direct } \\
\text { Experience } \\
\text { with NSSI }\end{array}$ & $\begin{array}{c}\text { Experience } \\
\text { with Mental } \\
\text { Health } \\
\text { Disorders }\end{array}$ & $\begin{array}{l}\text { Professional's } \\
\text { General } \\
\text { Knowledge of } \\
\text { NSSI }\end{array}$ & $\begin{array}{c}\text { Professional's } \\
\text { Perceived Self } \\
\text { Efficacy }\end{array}$ \\
\hline $\begin{array}{c}\text { Role of } \\
\text { Professional }\end{array}$ & 1.00 & & & & \\
\hline $\begin{array}{c}\text { Direct } \\
\text { Experience } \\
\text { with NSSI }\end{array}$ & + or - * $^{*}$ & 1.00 & & & \\
\hline $\begin{array}{c}\text { Experience } \\
\text { with Mental } \\
\text { Health } \\
\text { Disorders }\end{array}$ & + or $-^{*}$ & + & 1.00 & & \\
\hline $\begin{array}{l}\text { Professional's } \\
\text { General } \\
\text { Knowledge of } \\
\text { NSSI }\end{array}$ & + or - * $^{*}$ & + & + & 1.00 & \\
\hline $\begin{array}{l}\text { Professional's } \\
\text { Perceived Self } \\
\text { Efficacy }\end{array}$ & + or - * & + & + & + & 1.00 \\
\hline
\end{tabular}

*Direction of point-biserial correlation will depend on the role of school-based professional.

\section{Multivariate Analysis of Variance of Data (MANOVA)}

After the assumptions for MANOVA are examined, survey scores will be summed within survey sections for each participant. Afterwards, a k-group MANOVA will be performed on four dependent variables scores (direct experience with NSSI behaviors, familiarity with various mental health disorders, general knowledge of NSSI behavior score and perceived self-efficacy score) with role of school-based professional as the independent variable.

Multivariate, univariate, and post hoc tests will be reported and tested at the $p<$ .05 or less significance level. If the results are significant, univariate analyses will be conducted for each dependent variable to determine specifically where the significant 
effect is located. To control for Type I error associated with single multiple single ANOVAs, the Bonferroni correction method will be used. This method sets the p-value at .05 and conservatively calculates the new p-value by dividing it by the number of dependent variables (Stevens, 2001). Afterwards, post hoc tests are conducted to determine significant differences between school-based professional group (independent variables) means on dependent variables. The Tukey HSD statistic, a conservative procedure to control for family-wise error for all pairwise comparisons, will be used for post hoc analyses (Keppel \& Wickens, 2004).

Partial eta squared $\left(\eta_{\mathrm{p}}{ }^{2}\right)$ will be reported as proportions of sample variances accounted for by dependent variables associated with various groups within the independent variable. Partial eta squared considers all independent variables and interactions and partials out the variance accounted for by the independent variable (or group) of focus (Richardson, 2011). In other words, it is a method to account for sources of variance, other than error, to provide a more accurate estimate of variance. When reporting partial eta squared effect sizes, Cohen (1988) states that .0099 is a small effect size, .0588 is a medium effect size and .1379 is a large effect size.

\section{Linear Regression and Path Analysis}

Independent and dependent variables will be entered into models accordingly and path coefficients will be calculated using linear regression and path analytic techniques (Pedhazurh, 1997). The current study's model will be specified and approximated with IBM SPSS 21.0 program. To illustrate mediation, direct effects, and indirect effects used in the analysis, models are presented with dependent or endogenous variables with 
school-based professional's role serving as the independent or exogenous variable. Mediating variables, direct experience with NSSI behaviors and familiarity with various mental health disorders serve as predictor variables in when analyzing indirect effects on outcome variables general knowledge of NSSI behaviors and perceived self-efficacy of school-based professionals.

Role of school-based professional encompasses the following self-identified professions: school counselor, school nurse, school psychologist, school psychological examiner, general education teacher, special education teacher, school social worker, school/district administrator, and other support staff (e.g., media specialists, speechpathologists, administrative assistants). Given the significantly unequal sample sizes (n) across some the categories, roles will be collapsed into 7 distinct categories for analysis. These groups are general education teachers, special education teachers, school nurses, school social workers, mental health/counseling associates, support staff, and school/district administrators. For regression analysis, role of school-based professional will be dummy coded into 6 categories with general education teachers serving as the reference variable.

The enter selection technique for linear regression and path analysis will be employed to determine how well school-based professional's direct experience with students that engage in NSSI behaviors and familiarity with various mental health disorders explains school-based professional's general knowledge of NSSI and their perceived self-efficacy. If mediation or partial mediation is observed, indirect path coefficients will be significant and larger than direct path coefficients. Sobel tests will be 
calculated to determine if indirect effects are significant. See linear regression and path analysis assumptions for further details regarding Sobel test. 


\section{CHAPTER FIVE}

RESULTS

The purpose of the study is to determine school-based health and mental health professionals' knowledge of self-injury and relevant interventions. A k-Group Multivariate Analysis of Variance (MANOVA) was conducted on four dependent variables (general knowledge, perceived self-efficacy, direct experiences with NSSI, and familiarity with mental health disorders) with respondents' role in the school (school nurses, mental health and counseling associates, general education teachers, special education teachers, school social workers, support staff, and school/district administrators) as the independent variable.

A path analysis was conducted to determine the relationships among independent (predictor) variables, mediators, and dependent (outcome) variables. The independent variable in the model is role of the school-based professional. Mediating variables are professionals' direct experience with NSSI behaviors and familiarity with various mental health disorders. The dependent variables are general knowledge of NSSI and perceived self-efficacy scores.

\section{Overall Scores for Respondents on Dependent Variables}

The overall subscale scores and respective standard deviations for all respondents based upon their role in the schools for each dependent variable are reported in Tables 7 , 8,9 and 10 . Scores were calculated by summing the respective value for each response within subscales. Higher scores denote more general knowledgeable of NSSI behaviors for professionals. Additionally, higher perceived self-efficacy scores indicate that 
professionals perceive themselves as competent to work with students that engage in NSSI behaviors. Actual number of disorders that the professional is familiar with when working with students is presented. If professionals indicated other disorders than those presented in the survey, credit for each mental health disorder listed was given. The direct experience variable was on an increasing scale of "None to Very Regular Experiences" in working with students that engage in NSSI behaviors. 
Table 7

Range, Mean and Standard Deviation of Dependent Variable Scores by Professional's Role in the School

\begin{tabular}{|c|c|c|c|c|}
\hline \multirow[b]{2}{*}{$\begin{array}{c}\text { School-based } \\
\text { Professional's Role } \\
(\mathrm{n}=333)\end{array}$} & \multicolumn{4}{|c|}{ Dependent Variables } \\
\hline & $\begin{array}{c}\text { General } \\
\text { Knowledge } \\
\text { Score } \\
(\text { Min }=0.00, \\
\text { Max 54.00) } \\
\end{array}$ & $\begin{array}{c}\text { Direct Experience } \\
\text { with NSSI } \\
(\operatorname{Min}=0.00 \\
\operatorname{Max}=5.00)\end{array}$ & $\begin{array}{c}\text { Number of } \\
\text { Disorders } \\
\text { Familiar With } \\
\text { (Value varies } \\
\text { by experience) }\end{array}$ & $\begin{array}{c}\text { Perceived } \\
\text { Self-Efficacy } \\
\text { Score } \\
(\operatorname{Min}=0.00, \\
\text { Max }=40.00)\end{array}$ \\
\hline \multicolumn{5}{|l|}{$\begin{array}{l}\text { Regular Ed Teacher } \\
(\mathrm{n}=131)\end{array}$} \\
\hline Range & 39.00 & 4.00 & 9.00 & 33.00 \\
\hline Mean & 37.92 & 1.08 & 4.28 & 26.23 \\
\hline $\mathrm{SD}$ & 5.90 & .94 & 2.50 & 5.56 \\
\hline \multicolumn{5}{|l|}{$\begin{array}{l}\text { Special Ed Teacher } \\
(n=36)\end{array}$} \\
\hline Range & 22.00 & 4.00 & 11.00 & 36.00 \\
\hline Mean & 36.94 & 1.11 & 4.86 & 24.28 \\
\hline SD & 5.74 & 1.09 & 2.64 & 9.42 \\
\hline \multicolumn{5}{|l|}{$\begin{array}{l}\text { School Social Worker } \\
(\mathrm{n}=72)\end{array}$} \\
\hline Range & 23.00 & 4.00 & 10.00 & 17.00 \\
\hline Mean & 41.31 & 2.50 & 6.24 & 33.18 \\
\hline $\mathrm{SD}$ & 6.06 & 1.02 & 2.03 & 3.56 \\
\hline \multicolumn{5}{|l|}{$\begin{array}{l}\text { School Nurse } \\
(\mathrm{n}=30)\end{array}$} \\
\hline Range & 29.00 & 4.00 & 12.00 & 30.00 \\
\hline Mean & 37.63 & 1.67 & 6.13 & 28.10 \\
\hline $\mathrm{SD}$ & 6.34 & .99 & 2.05 & 9.25 \\
\hline \multicolumn{5}{|l|}{$\begin{array}{l}\text { Mental Health and } \\
\text { Counseling Associate } \\
(\mathrm{n}=22)\end{array}$} \\
\hline Range & 44.00 & 3.00 & 11.00 & 29.00 \\
\hline Mean & 38.36 & 2.09 & 6.50 & 31.59 \\
\hline $\mathrm{SD}$ & 9.06 & .68 & 2.11 & 5.90 \\
\hline \multicolumn{5}{|l|}{$\begin{array}{l}\text { Support Staff } \\
(\mathrm{n}=27)\end{array}$} \\
\hline Range & 31.00 & 4.00 & 8.00 & 35.00 \\
\hline Mean & 35.63 & .89 & 3.11 & 21.44 \\
\hline SD & 7.03 & 1.01 & 2.45 & 9.34 \\
\hline \multicolumn{5}{|l|}{$\begin{array}{l}\text { School/District } \\
\text { Administrator } \\
(\mathrm{n}=15)\end{array}$} \\
\hline Range & 24.00 & 4.00 & 6.00 & 40.00 \\
\hline Mean & 33.07 & 1.93 & 6.00 & 28.27 \\
\hline $\mathrm{SD}$ & 8.22 & 1.16 & 1.96 & 9.25 \\
\hline
\end{tabular}


Table 8

Range, Mean, and Standard Deviation of Dependent Variable Scores by Professional's School Assignment (60\% or more time spent)

\begin{tabular}{|c|c|c|c|c|}
\hline \multirow[b]{2}{*}{$\begin{array}{c}\text { School-Based } \\
\text { Professional } \\
\text { District Placement } \\
(\mathrm{n}=333)\end{array}$} & \multicolumn{4}{|c|}{ Dependent Variables } \\
\hline & $\begin{array}{c}\text { General } \\
\text { Knowledge } \\
\text { Score } \\
(\text { Min }=0.00, \\
\text { Max 54.00) }\end{array}$ & $\begin{array}{c}\text { Direct } \\
\text { Experience with } \\
\text { NSSI } \\
(\operatorname{Min}=0.00, \\
\operatorname{Max}=5.00) \\
\end{array}$ & $\begin{array}{c}\text { Number of } \\
\text { Disorders } \\
\text { Familiar With } \\
\text { (Value varies by } \\
\text { experience) }\end{array}$ & $\begin{array}{c}\text { Perceived } \\
\text { Self-Efficacy } \\
\text { Score } \\
(\operatorname{Min}=0.00, \\
\operatorname{Max}=40.00)\end{array}$ \\
\hline \multicolumn{5}{|l|}{ Preschool/Early } \\
\hline \multicolumn{5}{|l|}{ Childhood $(n=12)$} \\
\hline Range & 24.00 & 2.00 & 11.00 & 36.00 \\
\hline Mean & 36.42 & .50 & 3.67 & 23.92 \\
\hline $\mathrm{SD}$ & 7.53 & .80 & 3.26 & 9.91 \\
\hline \multicolumn{5}{|l|}{$\begin{array}{l}\text { Elementary } \\
(\mathrm{n}=128)\end{array}$} \\
\hline Range & 33.00 & 4.00 & 11.00 & 40.00 \\
\hline Mean & 38.37 & 1.17 & 4.69 & 27.47 \\
\hline SD & 5.85 & .99 & 2.35 & 7.07 \\
\hline \multicolumn{5}{|l|}{$\begin{array}{l}\text { Middle School } \\
(\mathrm{n}=82)\end{array}$} \\
\hline Range & 28.00 & 4.00 & 13.00 & 31.00 \\
\hline Mean & 38.18 & 1.63 & 5.18 & 27.78 \\
\hline $\mathrm{SD}$ & 5.85 & 1.07 & 2.61 & 6.10 \\
\hline \multicolumn{5}{|l|}{$\begin{array}{l}\text { Junior High School } \\
(\mathrm{n}=5)\end{array}$} \\
\hline Range & 4.00 & 2.00 & 4.00 & 5.00 \\
\hline Mean & 46.40 & 2.60 & 6.60 & 33.20 \\
\hline $\mathrm{SD}$ & 1.52 & .89 & 1.67 & 2.17 \\
\hline \multicolumn{5}{|l|}{$\begin{array}{l}\text { High School } \\
(n=94)\end{array}$} \\
\hline Range & 47.00 & 4.00 & 12.00 & 40.00 \\
\hline Mean & 37.86 & 1.97 & 5.49 & 28.53 \\
\hline $\mathrm{SD}$ & 7.94 & 1.20 & 2.54 & 7.87 \\
\hline \multicolumn{5}{|l|}{$\begin{array}{l}\text { Other Combined } \\
(n=12)\end{array}$} \\
\hline Range & 23.00 & 4.00 & 9.00 & 24.00 \\
\hline Mean & 36.08 & 2.00 & 5.58 & 26.00 \\
\hline SD & 7.97 & 1.28 & 2.91 & 7.69 \\
\hline
\end{tabular}

a. Includes participants who endorsed responses that did not fit in the above school level placement category such as those working in buildings with all grades or district offices. 
Table 9

Range, Mean, and Standard Deviation of Dependent Variable Scores by Professionals' Highest Attained Degree

\begin{tabular}{|c|c|c|c|c|}
\hline \multirow[b]{2}{*}{$\begin{array}{c}\text { Highest Degree } \\
\text { Attained } \\
(\mathrm{n}=332,1 \text { missing })\end{array}$} & \multicolumn{4}{|c|}{ Dependent Variables } \\
\hline & $\begin{array}{c}\text { General } \\
\text { Knowledge } \\
\text { Score } \\
(\text { Min }=0.00 \\
\text { Max 54.00) }\end{array}$ & $\begin{array}{c}\text { Direct } \\
\text { Experience } \\
\text { with NSSI } \\
(\mathrm{Min}=0.00 \\
\operatorname{Max}=5.00)\end{array}$ & $\begin{array}{c}\text { Number of } \\
\text { Disorders } \\
\text { Familiar With } \\
\text { (Value varies by } \\
\text { experience) }\end{array}$ & $\begin{array}{c}\text { Perceived } \\
\text { Self-Efficacy } \\
\text { Score } \\
(\operatorname{Min}=0.00, \\
\operatorname{Max}=40.00)\end{array}$ \\
\hline \multicolumn{5}{|l|}{ High School } \\
\hline \multicolumn{5}{|l|}{ Diploma $(n=8)$} \\
\hline Range & 20.00 & 4.00 & 8.00 & 25.00 \\
\hline Mean & 29.88 & 1.00 & 2.38 & 14.25 \\
\hline $\mathrm{SD}$ & 6.94 & 1.41 & 3.07 & 7.87 \\
\hline \multicolumn{5}{|l|}{$\begin{array}{l}\text { Associates } \\
(\mathrm{n}=13)\end{array}$} \\
\hline Range & 27.00 & 3.00 & 8.00 & 26.00 \\
\hline Mean & 37.38 & 1.08 & 4.15 & 27.77 \\
\hline SD & 7.39 & 1.04 & 2.70 & 6.14 \\
\hline \multicolumn{5}{|l|}{$\begin{array}{l}\text { Bachelors } \\
(n=74)\end{array}$} \\
\hline Range & 30.00 & 4.00 & 12.00 & 38.00 \\
\hline Mean & 38.05 & 1.05 & 4.46 & 26.12 \\
\hline $\mathrm{SD}$ & 5.54 & .98 & 2.74 & 6.47 \\
\hline \multicolumn{5}{|l|}{$\begin{array}{l}\text { Masters } \\
(n=203)\end{array}$} \\
\hline Range & 47.00 & 4.00 & 13.00 & 40.00 \\
\hline Mean & 38.77 & 1.67 & 5.24 & 28.43 \\
\hline $\mathrm{SD}$ & 6.80 & 1.15 & 2.40 & 7.13 \\
\hline \multicolumn{5}{|l|}{$\begin{array}{l}\text { Specialist } \\
(\mathrm{n}=24)\end{array}$} \\
\hline Range & 25.00 & 4.00 & 8.00 & 23.00 \\
\hline Mean & 37.29 & 2.04 & 6.25 & 30.92 \\
\hline $\mathrm{SD}$ & 6.99 & 1.00 & 1.98 & 5.98 \\
\hline \multicolumn{5}{|l|}{$\begin{array}{l}\text { Doctorate } \\
(n=10)\end{array}$} \\
\hline Range & 17.00 & 4.00 & 5.00 & 13.00 \\
\hline Mean & 36.60 & 2.20 & 6.70 & 29.10 \\
\hline SD & 6.33 & 1.23 & 1.70 & 5.20 \\
\hline
\end{tabular}


Table 10

Range, Mean, and Standard Deviation of Dependent Variable Scores by Professionals' Region of the U.S. Worked

\begin{tabular}{|c|c|c|c|c|}
\hline \multirow[b]{2}{*}{$\begin{array}{l}\text { Region of the U.S. } \\
\qquad(\mathrm{n}=333)\end{array}$} & \multicolumn{4}{|c|}{ Dependent Variables } \\
\hline & $\begin{array}{c}\text { General } \\
\text { Knowledge } \\
\text { Score } \\
(\text { Min }=0.00, \\
\text { Max 54.00) }\end{array}$ & $\begin{array}{c}\text { Direct } \\
\text { Experience } \\
\text { with NSSI } \\
(\operatorname{Min}=0.00, \\
\operatorname{Max}=5.00)\end{array}$ & $\begin{array}{c}\text { Number of } \\
\text { Disorders } \\
\text { Familiar With } \\
\text { (Value varies by } \\
\text { experience) }\end{array}$ & $\begin{array}{c}\text { Perceived } \\
\text { Self-Efficacy } \\
\text { Score } \\
(\operatorname{Min}=0.00, \\
\operatorname{Max}=40.00)\end{array}$ \\
\hline \multicolumn{5}{|l|}{ North $(n=9)$} \\
\hline Range & 18.00 & 2.00 & 8.00 & 27.00 \\
\hline Mean & 40.33 & 1.56 & 4.56 & 26.22 \\
\hline $\mathrm{SD}$ & 6.14 & .88 & 2.79 & 10.24 \\
\hline \multicolumn{5}{|l|}{ South $(n=18)$} \\
\hline Range & 22.00 & 4.00 & 8.00 & 11.00 \\
\hline Mean & 40.78 & 2.67 & 5.94 & 32.50 \\
\hline $\mathrm{SD}$ & 5.33 & .97 & 2.07 & 3.33 \\
\hline \multicolumn{5}{|l|}{ East $(n=10)$} \\
\hline Range & 20.00 & 4.00 & 6.00 & 14.00 \\
\hline Mean & 38.10 & 2.20 & 5.70 & 32.60 \\
\hline $\mathrm{SD}$ & 8.16 & 1.32 & 1.95 & 4.20 \\
\hline \multicolumn{5}{|l|}{ West $(n=13)$} \\
\hline Range & 20.00 & 4.00 & 5.00 & 23.00 \\
\hline Mean & 39.54 & 2.23 & 6.23 & 32.62 \\
\hline $\mathrm{SD}$ & 6.85 & 1.01 & 1.92 & 5.94 \\
\hline \multicolumn{5}{|l|}{ Midwest $(n=283)$} \\
\hline Range & 47.00 & 4.00 & 13.00 & 40.00 \\
\hline Mean & 37.85 & 1.41 & 4.94 & 27.10 \\
\hline $\mathrm{SD}$ & 6.68 & 1.11 & 2.59 & 7.19 \\
\hline
\end{tabular}

\section{Survey Reliability}

Internal consistency reliability estimates for the original distinct sections of the measure yielded a Cronbach's Alpha ( $\left.{ }^{\alpha}\right)$ of .612 for section 2 (General Knowledge of NSSI Behaviors), and .873 for section 4 (School-Based Professionals' Perceived SelfEfficacy). An item reliability analysis was performed for each of the aforementioned sections of the survey. Using the SPSS reliability item analysis, results indicated no significant improvements $(\Delta=.001-.008)$ in reliability if various items were deleted as 
flagged by the statistical software as items that lowered the overall subsection's reliability. This observation was applicable to the general knowledge subsection of the instrument. Finally, the perceived self-efficacy subsection resulted in no significant improvements ( $\Delta=.006-.008)$ to Cronbach's Alpha coefficient.

\section{Multivariate Analysis of Variance (MANOVA) Assumptions.}

Prior to analyzing the data with MANVOA techniques, various assumptions were tested. The following assumptions were examined to determine the feasibility of using MANOVA to compare group differences for significance across all dependent variables. Independence of Observations. The IBM SPSS 21.0 procedure, General Linear Model, was used for the analyses of assumptions. The data reasonably satisfied the assumption of independent observations. The study assumes that participants logged onto the website and independently completed the survey. Professionals' scores on dependent variables appear to not be affected by others in the same group.

Normal Distribution of Dependent Variables. When determining if the data are normally distributed, multivariate and univariate normality tests were performed. Tables 11 and 12 report skewness and kurtosis values for dependent variables and role of professional by dependent variables, respectively. Using skewness and kurtosis statistics, multivariate tests of normality determined that the skewness and kurtosis maximums were not exceeded; therefore, the data are reasonably normally distributed. However, upon examination of univariate tests of normality, histograms and skewness and kurtosis statistics indicated that the data exhibited low to severe levels of skewness and kurtosis for various variables for regular education teachers, special education teachers, support 
staff, school nurses, school and district administrators, and mental health and counseling associates. When examining the dependent variable general knowledge of NSSI behaviors, skewness (-2.774) and kurtosis (9.957) maximum values were exceeded for mental health and counseling associates. Next, school nurses exceeded the skewness maximum value (-1.203) for general knowledge of NSSI behaviors. Skewness (-1.536) and kurtosis (4.777) maximum values were exceeded for regular education teachers within the general knowledge of NSSI behaviors section as well.

When examining the dependent variable school-based professional's perceived self-efficacy, skewness (-1.393) and kurtosis (5.605) maximum values were exceeded for school nurses. Skewness maximum values were slightly exceeded for special education teachers

$(-1.029)$ and moderately exceeded the maximum value for mental health and counseling associates (-2.336). Kurtosis maximum values were exceeded for mental health and counseling associates (8.269) and school/district administrators (6.363).

When examining dependent variable direct experience with NSSI behaviors, skewness maximum values for support staff (1.197) were exceeded. No kurtosis maximum values were exceeded for direct experience with NSSI behaviors.

Finally, when examining the dependent variable familiarity with various mental health disorders, skewness maximum values were slightly exceeded for school/district administrators (-1.043). Kurtosis maximum values were exceeded for the following school-based professionals: school nurses (3.878) and mental health and counseling associates (3.828). 


\section{Table 11}

Multivariate Skewness and Kurtosis Values for Dependent Variables Before Transformation Methods

\begin{tabular}{ccccc}
\hline & \multicolumn{4}{c}{ Dependent Variables } \\
\cline { 2 - 4 } & General & Direct & Number of & Perceived \\
Knowledge & Experience & Disorders & Familiar to the \\
Test of Normality & Score & with NSSI & $\begin{array}{c}\text { Self-Efficacy } \\
\text { Professional }\end{array}$ & Score \\
\hline
\end{tabular}

Skewness

Value

$-1.123 *$

.371

$-.238$

$-1.183 *$

Kurtosis

Value

2.861

$-.531$

$-.333$

2.173

*Exceeds maximum skewness value for acceptable range of normality $( \pm 1)$. ** Exceeds maximum kurtosis value for acceptable range of normality $( \pm 3)$. 
Table 12

Univariate Test of Skewness and Kurtosis for Role of School-Based Profession by Dependent Variable Before Transformation Methods

\begin{tabular}{|c|c|c|c|c|}
\hline \multirow[b]{2}{*}{$\begin{array}{c}\text { School-based } \\
\text { Professional's Role } \\
(\mathrm{n}=333)\end{array}$} & \multicolumn{4}{|c|}{ Dependent Variables } \\
\hline & $\begin{array}{l}\text { General } \\
\text { Knowledge } \\
\text { Score }\end{array}$ & $\begin{array}{c}\text { Direct Experience } \\
\text { with NSSI }\end{array}$ & $\begin{array}{l}\text { Number of } \\
\text { Disorders } \\
\text { Familiar to the } \\
\text { Professional } \\
\end{array}$ & $\begin{array}{c}\text { Perceived } \\
\text { Self-Efficacy } \\
\text { Score }\end{array}$ \\
\hline \multicolumn{5}{|l|}{$\begin{array}{l}\text { Regular Ed Teacher } \\
(\mathrm{n}=131)\end{array}$} \\
\hline Skewness & $-1.536^{*}$ & .738 & -.084 & -.390 \\
\hline Kurtosis & $4.777 * *$ & .540 & -1.043 & 1.154 \\
\hline \multicolumn{5}{|l|}{$\begin{array}{l}\text { Special Ed Teacher } \\
(\mathrm{n}=36)\end{array}$} \\
\hline Skewness & -.618 & .751 & -.067 & $-1.029 *$ \\
\hline Kurtosis & -.300 & -.084 & -.024 & .667 \\
\hline \multicolumn{5}{|l|}{$\begin{array}{l}\text { School Social Worker } \\
(\mathrm{n}=72)\end{array}$} \\
\hline Skewness & -.462 & -.245 & -.269 & -.391 \\
\hline Kurtosis & -.905 & -.415 & -.077 & -.038 \\
\hline \multicolumn{5}{|l|}{$\begin{array}{l}\text { School Nurse } \\
(\mathrm{n}=30)\end{array}$} \\
\hline Skewness & $-1.203 *$ & -.159 & -.348 & $-1.393 *$ \\
\hline Kurtosis & 2.559 & .087 & $3.878^{* *}$ & $5.605^{* *}$ \\
\hline \multicolumn{5}{|l|}{$\begin{array}{l}\text { Mental Health and } \\
\text { Counseling Associate } \\
(n=22)\end{array}$} \\
\hline Skewness & $-2.774 *$ & .869 & .937 & $-2.336^{*}$ \\
\hline Kurtosis & $9.957 * *$ & 2.199 & $3.828 * *$ & $8.269 * *$ \\
\hline \multicolumn{5}{|l|}{$\begin{array}{l}\text { Support Staff } \\
(\mathrm{n}=27)\end{array}$} \\
\hline Skewness & -.624 & $1.197^{*}$ & .333 & -.495 \\
\hline Kurtosis & .805 & 1.702 & -.950 & -.605 \\
\hline \multicolumn{5}{|l|}{$\begin{array}{l}\text { School/District } \\
\text { Administrator } \\
(\mathrm{n}=15)\end{array}$} \\
\hline Skewness & .228 & .461 & $-1.044 *$ & $-2.099 *$ \\
\hline Kurtosis & -1.328 & -.354 & .530 & $6.363 * *$ \\
\hline
\end{tabular}

Shapiro-Wilks tests indicate a departure from normality for direct experience when working with NSSI behaviors for all school-based professionals except school/district administrators $(\mathrm{p}<.05)$. Additionally, Shapiro-Wilks tests indicate a 
violation of normality for familiarity with various mental health disorders for regular education teachers, school nurses, school/district administrators, support staff, and mental health/counseling associates $(\mathrm{p}<.05)$. Next, the Shapiro-Wilks test indicated a departure from normality for general knowledge of NSSI behaviors specifically for regular education teachers, school social workers, and mental health and counseling associates ( $p$ $<.05)$. Finally, Shapiro-Wilks tests indicated a departure from normality for the dependent variable perceived self-efficacy scores for school social workers, school/district administrators, and mental health/counseling associates $(\mathrm{p}<.05)$. Nonetheless, the F test is robust to departures from normality so long as the data do not demonstrate platykurtosis (i.e., a flat and wide distribution shape) as illustrated through histograms plots (T. Whittaker, Class notes, September 2005; Stevens, 2001). After examining histogram plots across dependent variables by role, no distribution appeared platykurtotic and therefore can reasonably be included in the final analysis.

Univariate tests of normality indicate dependent variables familiarity with various mental health disorders and perceived self-efficacy is seriously non-normally distributed across numerous roles of school-based professionals. Due to normality violations, data transformation methods were employed. Final skewness and kurtosis values for dependent variables after transformation methods are presented in Table 13, 14, and 15. 
Table 13

Multivariate Skewness and Kurtosis Values for Dependent Variables After Transformation Methods

\begin{tabular}{lcccc}
\hline & \multicolumn{4}{c}{ Dependent Variables } \\
\cline { 2 - 5 } & $\begin{array}{c}\text { General } \\
\text { Knowledge } \\
\text { Score }\end{array}$ & $\begin{array}{c}\text { Direct } \\
\text { Experience } \\
\text { with NSSI }\end{array}$ & $\begin{array}{c}\text { Number of } \\
\text { Disorders } \\
\text { Familiar to the } \\
\text { Professional }\end{array}$ & $\begin{array}{c}\text { Pelf-Efficacy } \\
\text { Score }\end{array}$ \\
\hline $\begin{array}{l}\text { Skewness } \\
\text { Value }\end{array}$ & -.653 & .371 & -.238 & -.376 \\
$\begin{array}{l}\text { Kurtosis } \\
\text { Value }\end{array}$ & .612 & -.531 & -.333 & -.186 \\
& & & & \\
\hline
\end{tabular}

*Exceeds maximum skewness value for acceptable range of normality $( \pm 1)$. ** Exceeds maximum kurtosis value for acceptable range of normality $( \pm 3)$.

Transformation Procedure to Satisfy Normality Assumption. Transformation methods were performed to satisfy the normality assumption needed for MANOVA and path analysis. Test of normality for questions in the General Knowledge of NSSI Behaviors and Perceived Self-Efficacy sections of the survey are listed in Tables 12 and 13. These tables provide skewness and kurtosis values before and after transformation methods were employed. The square root, inverse, $\log _{10}$, and natural log methods were examined. After exploring all transformation methods, the log base 10 and inverse methods improved normality of the dependent variable perceived self-efficacy. Reflection of most variables within the general knowledge of NSSI behaviors and selfefficacy mean scores was carried out prior to transformation. Reflection changes the direction of skewed variables so that they are positively and slightly skewed approaching normality. 
After transformation, some variables that violated maximum values were within limits of normality. It should be noted that while multivariate skewness statistics and graphs indicated no violations for the dependent variables familiarity with various mental health disorders and direct experience with NSSI behaviors after transformation methods, they still seriously violated normality, per Shapiro-Wilks statistics, skewness, and kurtosis statistics. Therefore, no transformation methods were employed on these variables. Conversely, normality was dramatically improved for the dependent variables perceived self-efficacy and general knowledge of NSSI behaviors after transformation methods were employed. This is to be expected given that the latter dependent variables are composed of multiple variables that are capable of being transformed. 
Table 14

Pre and Post Transformation Tests of Normality Values for Questions Within the General Knowledge of NSSI Behaviors Scale

\begin{tabular}{|c|c|c|c|c|c|}
\hline $\begin{array}{c}\text { General } \\
\text { Knowledge } \\
\text { Section Items }\end{array}$ & $\begin{array}{c}\text { Pre } \\
\text { Skewnes } \\
\text { s Value }\end{array}$ & $\begin{array}{c}\text { Pre } \\
\text { Kurtosis } \\
\text { Value }\end{array}$ & $\begin{array}{c}\text { Optimal } \\
\text { Transformation } \\
\text { Method }\end{array}$ & $\begin{array}{c}\text { Post } \\
\text { Skewness } \\
\text { Value }\end{array}$ & $\begin{array}{l}\text { Post } \\
\text { Kurtosis } \\
\text { Value }\end{array}$ \\
\hline Referred to as cutting & $-2.177^{*}$ & 2.943 & Reflect \& inverse & $-2.024 *$ & 2.194 \\
\hline Female populations & $-1.881^{*}$ & 1.725 & Reflect \& $\log _{10}$ & $1.792 *$ & 1.355 \\
\hline Male populations & $-2.366^{*}$ & $3.785 * *$ & Reflect \& inverse & $-2.233 *$ & $3.082 * *$ \\
\hline Coping Mechanism & -2.918 & 7.031 & Reflect \& inverse & $-2.627 *$ & $5.104^{* *}$ \\
\hline Enjoys physical pain & -.997 & -.740 & - & -.997 & -.740 \\
\hline Individuals with OCD & -.073 & -1.346 & - & -.073 & -1.346 \\
\hline Sexual Abuse & -.553 & -1.542 & - & -.553 & -1.542 \\
\hline Disorder in DSM & .065 & -.468 & - & .065 & -.468 \\
\hline Tattoo and piercings & -.918 & -.798 & - & -.918 & -.798 \\
\hline Rite of passage & -.364 & -1.332 & - & -.364 & -1.332 \\
\hline Attempt suicide & -.095 & -1.154 & - & -.095 & -1.154 \\
\hline Individual therapy & -.475 & -1.116 & - & -.475 & -1.116 \\
\hline Lower SES & -1.393 & .450 & Reflect \& $\log _{10}$ & $1.157^{*}$ & -.355 \\
\hline Ethnic groups & -2.187 & 3.264 & Reflect \& inverse & $-1.874^{*}$ & 1.669 \\
\hline Related to attachment & .572 & -1.006 & - & .572 & -1.006 \\
\hline Over 30 Observed & -.925 & -.902 & - & -.925 & -.902 \\
\hline Eating disorder & .230 & -1.032 & - & .230 & -1.032 \\
\hline Attention seeking & .114 & -1.147 & - & .114 & -1.147 \\
\hline School age population & -1.392 & .276 & Reflect \& inverse & $-1.108 *$ & -.661 \\
\hline Physical abuse & -.948 & -.930 & - & -.948 & -.930 \\
\hline More males & -1.494 & .658 & Reflect \& inverse & $-1.166^{*}$ & -.515 \\
\hline Emotional Abuse & -1.527 & .494 & Reflect \& inverse & $-1.377^{*}$ & -.034 \\
\hline Only in Caucasians & -2.762 & 6.499 & Reflect \& inverse & $-2.328 *$ & $3.654 * *$ \\
\hline Underlying symptom & -2.735 & 5.908 & Reflect \& inverse & $-2.477 *$ & $4.308 * *$ \\
\hline No other strategies & -1.877 & 1.763 & Reflect \& inverse & $-1.686^{*}$ & .943 \\
\hline $\mathrm{BPD}$ & -1.240 & .222 & Reflect \& $\log _{10}$ & .948 & -.741 \\
\hline Leads to suicide & -.402 & -1.422 & - & -.402 & -1.422 \\
\hline
\end{tabular}

*Exceeds maximum skewness value for acceptable range of normality $( \pm 1)$. ${ }^{* *}$ Exceeds maximum kurtosis value for acceptable range of normality $( \pm 3)$. 
Table 15

Pre and Post Transformation Tests of Normality Values for Questions Within Professional's Perceived Self-Efficacy Scale

\begin{tabular}{|c|c|c|c|c|c|}
\hline $\begin{array}{c}\text { General } \\
\text { Knowledge } \\
\text { Section Items }\end{array}$ & $\begin{array}{l}\text { Pre } \\
\text { Skewnes } \\
\text { s Value }\end{array}$ & $\begin{array}{l}\text { Pre } \\
\text { Kurtosi } \\
\text { s Value }\end{array}$ & $\begin{array}{c}\text { Optimal } \\
\text { Transformation } \\
\text { Method }\end{array}$ & $\begin{array}{c}\text { Post } \\
\text { Skewnes } \\
\text { s Value }\end{array}$ & $\begin{array}{c}\text { Post } \\
\text { Kurtosi } \\
\text { s Value }\end{array}$ \\
\hline $\begin{array}{l}\text { Comfortable } \\
\text { intervening }\end{array}$ & -.342 & -.636 & - & -.342 & -.636 \\
\hline $\begin{array}{l}\text { Comfortable with } \\
\text { recommendations }\end{array}$ & -.199 & -.737 & - & -.199 & -.737 \\
\hline $\begin{array}{l}\text { Comfortable } \\
\text { speaking with } \\
\text { students }\end{array}$ & -.934 & .346 & - & -.934 & .346 \\
\hline $\begin{array}{l}\text { Comfortable with } \\
\text { coping strategies }\end{array}$ & -.522 & -.391 & - & -.522 & -.391 \\
\hline $\begin{array}{l}\text { Comfortable with } \\
\text { crisis planning }\end{array}$ & -.167 & -.737 & - & -.167 & -.737 \\
\hline $\begin{array}{l}\text { Comfortable if knew } \\
\text { more about NSSI }\end{array}$ & $-1.384^{*}$ & 1.931 & Reflect \& $\log _{10}$ & .187 & -.579 \\
\hline $\begin{array}{l}\text { Comfortable on } \\
\text { multidisciplinary } \\
\text { teams }\end{array}$ & $-1.412^{*}$ & 1.929 & Reflect \& $\log _{10}$ & .167 & -.297 \\
\hline $\begin{array}{l}\text { Comfortable with } \\
\text { professional } \\
\text { development }\end{array}$ & $-1.725^{*}$ & $3.549 * *$ & Reflect \& inverse & .274 & -1.453 \\
\hline
\end{tabular}

Homogeneity of Variance-Covariance Matrices. Box's Test of Equality of Covariance Matrices did not support the assumption of homogeneity of covariance matrices $(\mathrm{p}<.01)$. Box's test it is highly sensitive to departures of normality such as those discussed in previous sections (Stevens, 2001). Similalry, homogeneity of variance for univariate $\mathrm{F}$ tests were significant for dependent variables familiarity with mental health disorders and perceived self-efficacy $(\mathrm{p}<.05)$ per Levene's Test of Equality of 
Error Variances. These findings suggest that greater error variances could be observed for larger groups of professionals resulting in a conservative $\mathrm{F}$ test. Additionally, this finding could also lead to rejecting the null hypothesis falsely more frequently. Statistical power may be adversely impacted as well. However, due to the number of groups that are included in the MANOVA analysis, a conservative p-value for statistical significance is utilized. This allows for the observance and interpretation of true group differences rather than significant findings due to sample size differences and higher error variances and covariances. Lastly, due to unequal variances assumed, Games-Howell statistics are reported for dependent variables that violated Levene's test.

\section{Table 16}

Expected Number of Participants for A Priori Power Analysis of Power $=.95$ (Needed $\mathrm{N}=330$ )

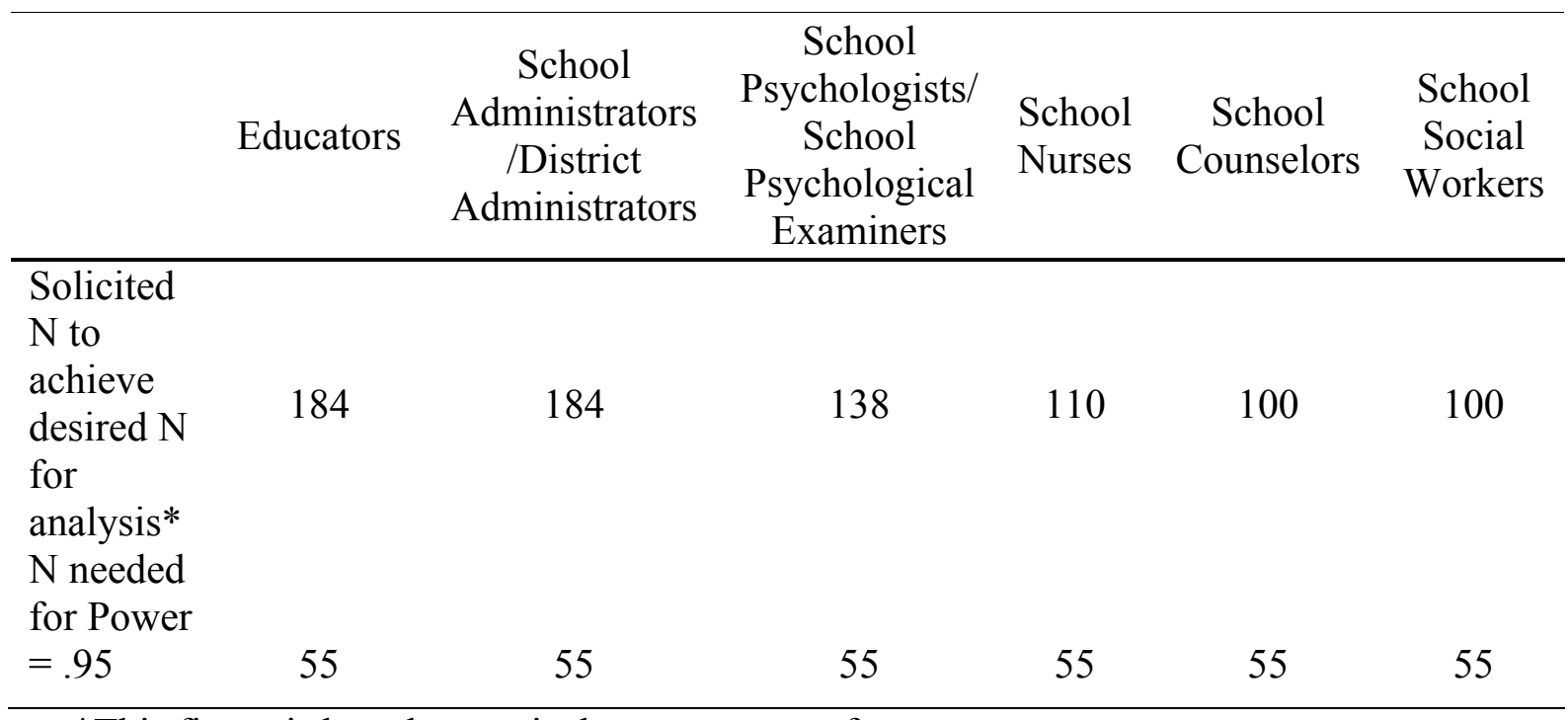

\footnotetext{
*This figure is based on typical response rates for surveys.
} 
A priori and Post Hoc Power Analysis for MANOVA

Table 16 presents the number of participants needed to achieve desired study power of .95. Upon further examination of data, larger variances were associated with large groups of professionals such as general education teachers and special education teachers. Although the F-test is conservative, power was extremely high (.965-1.00). The values of power for the multivariate analysis of professional's role in the school was 1.00 using Pillai's Trace statistic. There have been a number of researchers (Beasley \& Sheehan, 1994; Olson, 1976; Tabachnick \& Fidell, 2007) who advocate for the use of Pillai's Trace statistic over Wilks' Lambda when data is seriously non-normally distributed as evident with dependent variables general knowledge of NSSI behaviors and familiarity with mental health disorders. The statistic has proven to be robust against Type I error inflation (Beasley \& Sheehan, 1994). While some of the predicted number of subjects by profession was not met, the study far exceeded the number for general education teachers, special education teachers, and school social workers.

\section{Missing Values Analysis/Unequal Cell Sizes}

There were a relatively small number of missing values in the current data set. Little's Missing Completely At Random (MCAR) test was not significant $\left[\chi^{2}(74)=82.07\right.$, $\mathrm{P}>.05$ ]indicating that data was missing completely at random. Deleting all professionals with missing values was not an option as crucial data would be lost. The total number of professionals that began the survey was 361 . The conservative deletion of missing data involved identifying the number of participants in which outcome measures were not 
answered. A total of 28 surveys were deleted yielding a final number of 333 professionals in the current study.

Table 17 reports missing data patterns among professionals and specific questions within various sections of the survey. The data represented below are observed patterns of the 333 professionals in the study. Missing values were treated with the assumption that there are unequal sample sizes in all cells for the analysis. Although most researchers support the use of the Type III method when interpreting MANOVA findings, difficulty generalizing and interpreting findings is observed with data analysis methods when cell sizes are unequal (Tabachnik \& Fidell, 2007). The Type I method was selected to adjust for unequal cell sizes and allow the researcher to develop the current study model. Using Type I sums of squares is preferable with nonexperimental as well as controlling for main effects and interactions in the study model (Langsrud, 2003; Tabachnik \& Fidell, 2007). 


\section{Table 17}

Missing Value Patterns Among School-Based Professionals for the Knowledge of NSSI Behaviors and Perceived Self-Efficacy Survey Sections

\begin{tabular}{|c|c|c|}
\hline \multirow{2}{*}{$\begin{array}{l}\text { Role of School-Based } \\
\text { Professional }\end{array}$} & \multicolumn{2}{|c|}{$\begin{array}{c}\text { Number of Professionals with } \\
\text { Missing Responses Across } \\
\text { Outcome Measures (\%) }\end{array}$} \\
\hline & $\begin{array}{c}\text { General } \\
\text { Knowledge of } \\
\text { NSSI Behaviors }\end{array}$ & $\begin{array}{l}\text { School-Based } \\
\text { Professionals' } \\
\text { Self-Efficacy }\end{array}$ \\
\hline Regular Education Teacher $(\mathrm{N}=131)$ & 15 & 15 \\
\hline Special Education Teacher $(\mathrm{N}=36)$ & 4 & 4 \\
\hline School Social Worker $(\mathrm{N}=72)$ & 7 & 7 \\
\hline School Nurse $(\mathrm{N}=30)$ & 4 & 4 \\
\hline School/District Administrator $(\mathrm{N}=15)$ & 3 & 3 \\
\hline Support Staff $(\mathrm{N}=27)$ & 9 & 9 \\
\hline $\begin{array}{l}\text { Mental Health/ } \\
\text { Counseling Associate }(\mathrm{N}=22)\end{array}$ & 1 & 1 \\
\hline
\end{tabular}




\section{Multivariate Analysis of Variance Analysis (MANOVA) Interpretations}

MANOVA Research Question \#1A: Overall, is role of school based

professional significantly associated with knowledge of mental health disorders,

knowledge of NSSI behaviors, and experience with NSSI behaviors, and perceived self-efficacy working with students engaging in NSSI behaviors?

The results of the MANOVA analysis are shown in Table 18. There is a significant multivariate effect of school-based professionals' role in the school on the set of dependent variables, $F(24,1300)=.487, \mathrm{p}<.000$, which has a moderately large effect $\left(\eta_{\mathrm{p}}{ }^{2}=.12\right)$. This suggests that the study participant's role in the school significantly affected their responses regarding their knowledge of self-injurious behaviors, perceived self efficacy, direct experience with NSSI behaviors, and familiarity with mental health disorders, and accounting for $12 \%$ of the variance of scores.

Table 18

Multivariate Analysis: Effects of Role in School and School Placement on Four Dependent Variables

\begin{tabular}{lcccccc}
\hline Source & $\begin{array}{c}\text { Pillai's } \\
\text { Trace }\end{array}$ & $F$ & $\begin{array}{c}\text { Hypothesis } \\
d f\end{array}$ & $\begin{array}{c}\text { Error } \\
D f\end{array}$ & $p$ & Partial $\eta^{2}$ \\
\hline $\begin{array}{l}\text { Professional's } \\
\text { Role in the }\end{array}$ & & & & & & \\
School & .487 & 7.51 & 24 & 1300 & $<.000$ & .122 \\
\hline
\end{tabular}


Univariate Analysis of Variance

MANOVA Research Question \#1B: If there is a significant multivariate effect, is there a significant univariate effect of school-based professional roles on the dependent variables?

Results of the univariate analysis of variance are shown in Table 19. There is a significant univariate effect of school-based professionals' role in the schools on their direct experience with NSSI behaviors variable $(\mathrm{p}<.000)$, representing a large effect size on the dependent variable $\left(\eta_{\mathrm{p}}{ }^{2}=.28\right)$. This suggests that school-based professionals' role in the school significantly affected their general knowledge of NSSI behaviors. There is also a significant univariate effect of school-based professionals' role on their familiarity of mental health disorders variable $(p<.000)$, representing a large effect size on the dependent variable $\left(\eta_{p}{ }^{2}=.18\right)$. This suggests that school-based professionals' role in the school significantly affected their familiarity with mental health disorders. Similarly, significant univariate effects of school-based professionals' role in the school significantly affected their general knowledge of NSSI behaviors and perceived self efficacy (both at the $\mathrm{p}<.000$ ), representing medium to moderately large effect sizes on the dependent variable $\left(\eta_{\mathrm{p}}{ }^{2}=\right.$ $.06)$ and $\left(\eta_{\mathrm{p}}^{2}=.31\right)$, respectively. 


\section{Table 19}

Univariate Analyses: Effect of School-Based Professional's Role in School on Dependent Variables General Knowledge of NSSI Behaviors, Direct Experience with NSSI Behaviors, Familiarity with Mental Health Disorders, and Perceived Self-Efficacy with Transformed Scores

\begin{tabular}{lcccccc}
\hline Source & $S S^{a}$ & $d f$ & $M S$ & $F$ & $p$ & Partial $\eta^{2}$ \\
\hline $\begin{array}{l}\text { Direct Experience } \\
\text { with NSSI }\end{array}$ & & & & & & \\
Behaviors & & & & & & \\
$\quad$ Role in School & 120.86 & 6 & 20.14 & 20.14 & $<.000$ & .28 \\
$\quad$ Error & 313.71 & 325 & .97 & & & \\
Familiarity with & & & & & & \\
Mental Health & & & & & & \\
Disorders & & & & & \\
$\quad$ Role in School & 376.72 & 6 & 62.77 & 11.55 & $<.000$ & \\
$\quad$ Error & 1766.96 & 325 & 5.44 & & & \\
General Knowledge & & & & & & \\
of NSSI Behaviors & & & & & & \\
$\quad$ Role in School & 427.00 & 6 & 71.17 & 3.29 & $<.004$ & \\
$\quad$ Error & 7032.28 & 325 & 21.64 & & & \\
Perceived Self- & & & & & & \\
Efficacy & & & & & \\
$\quad$ Role in School & 3004.91 & 6 & 500.82 & 24.29 & $<.000$ & \\
$\quad$ Error & 6701.21 & 325 & 20.62 & & &
\end{tabular}


Post Hoc Tests

\section{MANOVA Research Question \#1C: Given a significant univariate effect, which} roles are significantly associated with each dependent variable?

Comparisons of means on dependent variables were conducted using the Tukey approach for dependent variables direct experience with NSSI behaviors and general knowledge of NSSI behaviors (Keppel \& Wickens, 2004). The Games-Howell procedure was used to examine pairwise differences between roles of school-based professionals for the familiarity with mental health disorders and perceived self-efficacy variables due to the violation of homogeneity of variance. Means and standard deviations for all four dependent variables are presented in Table 20. Due to having four dependent variables, I conservatively controlled for Type I error inflation by setting the alpha level at .007 . This was derived from dividing .05 by the number of groups in the independent variable school-based professionals' role in the school (i.e., $\mathrm{p}=.05 / 7=.007$ ). 
Table 20

Tukey HSD and Games-Howell Post Hoc Comparison of Mean Scores on Dependent Variables for School Based Professional's Role in the School (After Transformation Methods)

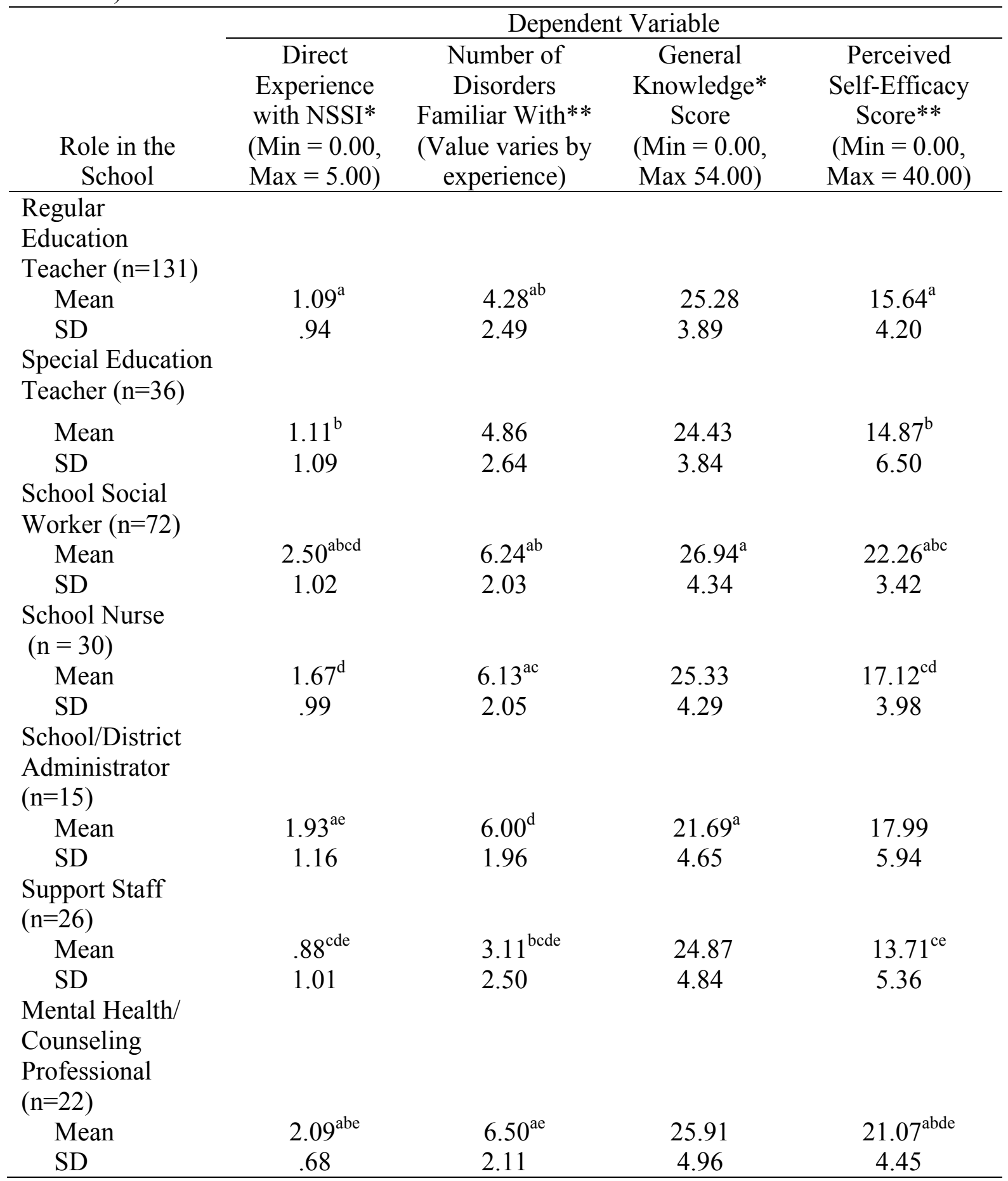

Notes. Means in the same column sharing the same letter superscript differ at $p<.007$. In addition, all means are reported before data transformation methods. *Tukey HSD statistic was used for mean comparisons. **Games-Howell statistic was used for mean comparisons. 
Direct Experience with NSSI Behaviors. Comparisons of means indicate a significant difference between school social workers and regular education teachers, special education teachers, school/district administrators, support staff, and school nurses with respect direct experience in working with students that engage in NSSI behaviors. This finding suggests that school social workers have significantly more direct experience working with students that engage in NSSI behaviors than regular education teachers, special education teachers, support staff, and school nurses. Similarly, mental health/counseling associates have significantly higher direct experience with NSSI behaviors than regular education teachers, special education teachers, school/district administrators, and support staff. This suggests that mental health/counseling associates have more direct experience working with students that engage in NSSI behaviors than support staff. Although not significantly different for all school-based professionals, school social workers had the highest self-reported experience working with students that engage in NSSI behaviors than any other professional.

Familiarity with Mental Health Disorders. Comparisons of means indicate a significant difference in the number of mental health disorders that professionals are familiar with through their work with students for support staff and most school-based professionals such as school social workers, school nurses, school/district administrators, and mental health/counseling associates. This means that support staff tended to have significantly less familiarity with various mental health disorders such as anxiety, depression, or eating disorders than their counterparts. Moreover, support staff had the least amount of familiarity of mental health disorders than all school-based professionals. Support staff was followed by regular education teachers with respect to lower number of 
familiarity with various mental health disorders. Regular education teachers reported significantly less familiarity with various mental health disorders than school social workers, school nurses, and mental health/counseling associates. Interestingly, special education teachers were not significantly different than any school-based professional.

General Knowledge of NSSI Behaviors. Mean comparisons indicate a significant difference in the amount of general knowledge between school social workers and school/district administrators. This finding suggests that school/district administrators have significantly less general knowledge of NSSI behaviors than school social workers. There were no other significant differences observed as most groups of professionals obtained scores that were close in range to one another. School social workers were observed to have the highest general knowledge scores overall.

Perceived Self-Efficacy. Finally, mean comparisons indicate a significant difference in the level of perceived self-efficacy between school social workers and regular education teachers, school nurses, support staff, and special education teachers. General education teachers, special education teachers and support staff tended to rate their perceived self-efficacy lower than school social workers. This suggests that school social workers may feel more at ease interacting and providing intervention to students that engage in NSSI behaviors than their peers. Similarly, mental health/counseling associates reported significantly greater levels of perceived self-efficacy than regular education teachers, special education teachers, school nurses, and support staff. Mental health/counseling associates tended to rate their perceived self-efficacy levels higher than most of their peers. It is striking that support staff rated themselves considerably lower in perceived self-efficacy, yet they scored similar general knowledge scores to regular and 
special education teachers and greater that school/district administrators. The implications of these findings are discussed in the following chapter.

\section{Bivariate Correlational Data}

Table 2 illustrates point-biserial correlations for all independent and dependent variables by professional's role. Dummy coding was employed on the independent variable with general education teachers serving as the reference group. 


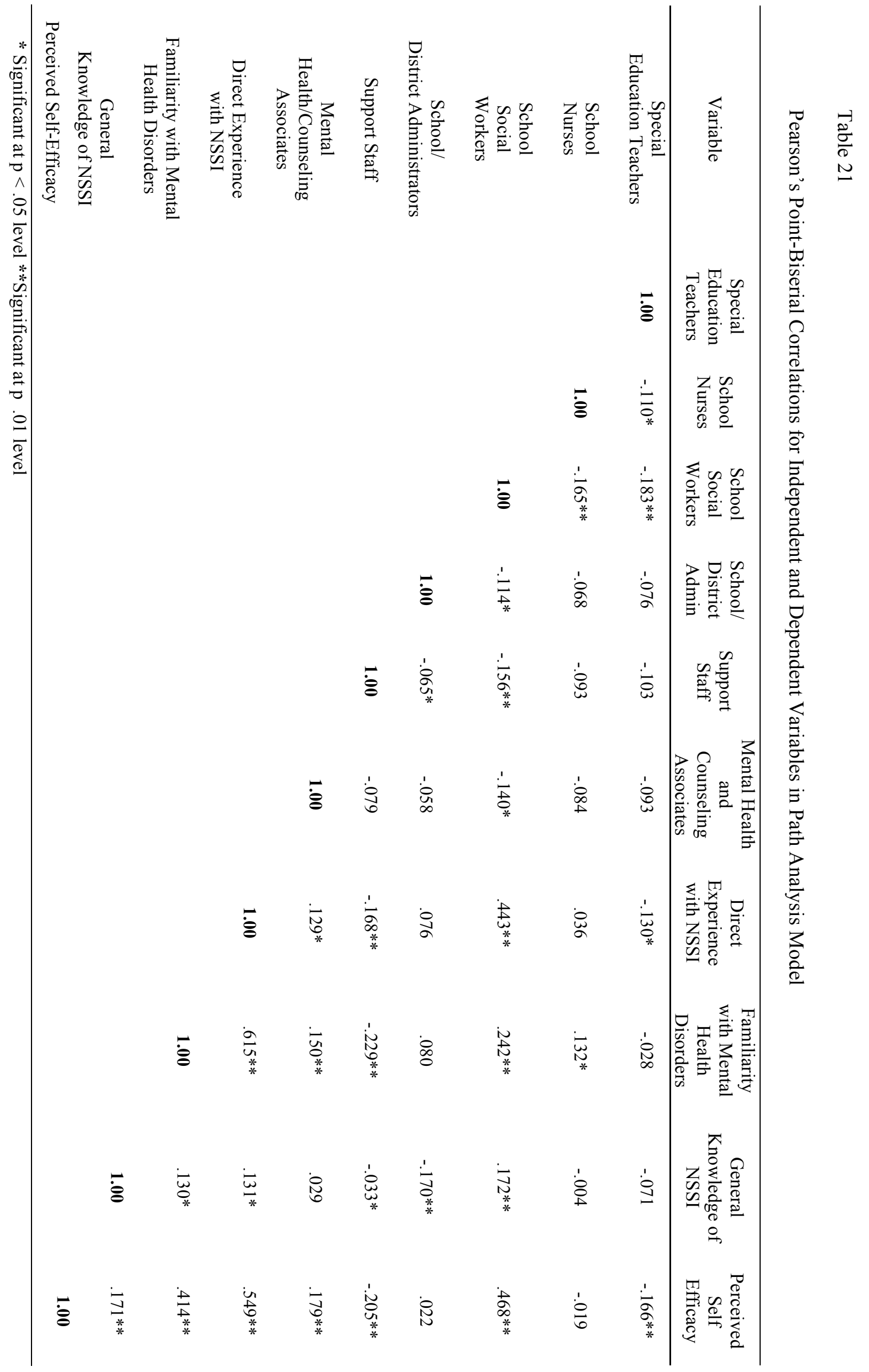


Perceived Self-Efficacy Bivariate Relationships. There is positive, moderate, and significant $(\mathrm{p}<.05)$ relationship between school Social Workers versus non-School Social Workers and perceived self-efficacy. School Social Workers tended to indicate higher levels of perceived self-efficacy than non-School Social Workers. There is also a negative, low, and significant $(\mathrm{p}<.01)$ relationship between Support Staff versus nonSupport Staff, Special Education Teachers and non-Special Education Teachers, and School Nurses versus non-School Nurses. These professionals were more likely to indicate lower levels of perceived self-efficacy than their counter parts.

General Knowledge of NSSI Behaviors Bivariate Relationships There is a positive, low, and significant $(\mathrm{p}<.01)$ relationship between School Social Workers versus non-School Social Workers and general knowledge of NSSI behaviors. School Social Workers tended to have more general knowledge of NSSI behaviors than nonSchool Social Workers. Conversely, there is a negative, low, and significant $(\mathrm{p}<.05)$ relationship between Support Staff versus non-Support Staff and general knowledge of NSSI behaviors. Support Staff tended to have less general knowledge of NSSI behaviors than non-Support Staff. Similar findings were observed with a negative, significant, and low relationship between School/District Administrators versus non-School/District Administrators.

Direct Experience with NSSI Behaviors Bivariate Relationships. There is a positive, moderate, and significant $(\mathrm{p}<.01)$ relationship between School Social Workers and non-School Social Workers and direct experience with NSSI behaviors. School Social Workers tended to have more direct experience with students that engage in NSSI behaviors. Similarly, positive, low, and significant $(\mathrm{p}<.05)$ relationship between Mental 
Health and Counseling Associates versus non-Mental Health Counseling Associates was observed. Mental Health and Counseling Associates tended to have more direct experience with students that engage in NSSI behaviors than non-Mental Health and Counseling Associates.

There is a negative, low, and significant $(\mathrm{p}<.01)$ relationship between Support Staff versus non-Support Staff and direct experience with NSSI behaviors. Support Staff tended to have less direct experience working with students that engage in NSSI behaviors. There is also a negative, low, and significant $(\mathrm{p}<.05)$ relationship between Special Education Teachers versus non-Special Education Teachers and direct experience with NSSI behaviors. Special Education teachers tended to have less direct experience with students that engage in NSSI behaviors than non-Special Education Teachers.

Familiarity with Various Mental Health Disorders Bivariate Relationships. There is a positive, low, and significant $(\mathrm{p}<.05)$ relationship between School Nurses versus non-School Nurses and familiarity with various mental health disorders. School Nurses tended to have more experience working with students with various mental health disorders. There is also a positive, low, and significant $(\mathrm{p}<.01)$ relationship between School Social Workers versus non-School Social Workers and familiarity with various mental health disorders. School Social Workers tended to have more experience working with students with various mental health disorders. Finally, there is a positive, low and significant $(\mathrm{p}<.01)$ relationship between Mental Health and Counseling Associates versus non-Mental Health Counseling Associates. Mental Health and Counseling Associates tended to have more experience working with students who have various mental health disorders than non-Mental Health and Counseling Associates. 
There are negative, low, and significant $(\mathrm{p}<.01)$ relationship observed between Support Staff versus non-Support Staff and familiarity with various mental health disorders. Support Staff tended to have less experience working with students who have various mental health disorders than non-Support Staff.

\section{Linear Regression and Path Analysis Assumptions}

Prior to conducting regression and path analytic techniques, a number of assumptions should be met to ensure the data meets and/or exceeds criteria to continue with analysis. These assumptions are accompanied by a brief explanation and relevant findings below.

Normal Distribution of Dependent Variables. This assumption overlaps with the normality findings obtained during MANOVA analysis. Multivariate tests of normality were not significant and therefore supported this assumption. However, two univariate tests were significant for dependent variables familiarity with various mental health disorders and general knowledge of NSSI behaviors. Because the data exhibited some levels of moderate to severe skewness, the model was estimated with maximum likelihood estimation. Additionally, general knowledge of NSSI behaviors was comprised of 27 individual variables that were reflected, and transformed resulting in sufficient univariate normality.

Linearity. Because the predictor variable is a forced dichotomous nominal variable, plotting the unstandardized residuals against the predictor variable and unstandardized predicted values is not meaningful. Therefore, linearity is not an 
applicable assumption in this study as predictor variables are typically on an interval level scale to offer valid tests.

Interval Level of Measurement for Dependent Variables. All variables used in the analysis are interval level. Variables that are used in the analysis are direct experience, familiarity with various mental health disorders, general knowledge of NSSI behaviors, and perceived self-efficacy. The direct experience variable is measured on an interval scale using the question, "Do you have direct experience in working with individuals who engage in NSSI behaviors?" Familiarity with various mental health disorders was placed on an interval scale measuring the number of distinct disorders in which the professional has experience working with students. It was measured with the question, "Please indicate the experience you have had with children related to the following mental health disorders." A comment box for the professional to indicate other disorders they are familiar with was included as well. They were appropriately given credit for the number of disorders listed. Both general knowledge of NSSI behaviors and perceived self-efficacy variables were measured using a number of questions in the second and fourth sections of the survey.

Lack of Measurement Error. Cronbach's alpha was used to estimate the reliability of responses on the study's instrument. Reliability coefficients ranged from .61 to .87 . Two sections fell well above .70 as the acceptable range for reliability. However the general knowledge scale fell outside the acceptable range and in the questionable range $(\alpha=.60-.69)$. Violation of this assumption for the general knowledge scale indicates path coefficient estimates should be interpreted with some degree of caution. 
Homoskedasticity. Testing the assumption of homeskedasticity of residuals is not applicable in this study as the independent variable, role of school-based professional, is a forced dichotomous, nominal variable. Results of plots would not be meaningful.

Lack of Multicollinearity. Multicollinearity was examined by calculating the squared correlation of the independent variable role of school-based professional. It was observed that none of the bivariate correlations for role of school-based professional exceeded .80 (Olobatuyi, 2006).

Model Specification. Theory and prior research supports the specification of the current model. The current causal ordering and selected variables is presumably correct and accurately depicted.

Model Identification. Using SPSS 21.0 statistical programming, the current model was determined to be just-identified. This signifies that there are unique parameter estimates for each path coefficient in the model.

Sobel Test of Significant Indirect Effects. The Sobel test is used to determine if the mediated path (i.e., indirect path) is significant. Baron and Kenny (1986), Preacher and Hayes (2008), and Sobel (1982) recommend the use of the Sobel test under the following conditions:

1. The independent variable significantly affects the mediator;

2. The independent variable significantly affects the dependent variable in the absence of mediators;

3. The mediator has a significant unique effect on the dependent variable;

4. The effect of the independent variable on the dependent variable shrinks upon the addition of the mediator. 
The following equation is a mathematical illustration of the how the Sobel test is calculated:

$$
\text { Sobel }(Z \text { value })=a \mathrm{X} \mathrm{b} / \mathrm{SQRT}\left(\mathrm{b}^{2} \mathrm{X} \mathrm{S}_{\mathrm{a}}{ }^{2}+\mathrm{a}^{2} \mathrm{X} \mathrm{S}_{\mathrm{b}}{ }^{2}\right)
$$

The Sobel test uses unstandardized regression coefficients ( $a$ and $b$ ) with their associated standard errors $\left(S_{a}\right.$ and $\left.S_{b}\right)$. Coefficient ' $a$ ' is the calculated path coefficient between the independent variable and the mediator. Coefficient ' $b$ ' is the calculated path coefficient between the mediator and the dependent variable.

\section{Linear Regression and Path Analysis Interpretations}

The hypothesized model is depicted in Figures 1-24. Rectangles represent measured exogenous and endogenous variables. Path estimates are displayed in the model. Straight, one headed arrows represent direct effects on the variables. Indirect effects are illustrated by a one-headed arrow to a mediating variable with another oneheaded arrow from the mediator connected to an outcome variable. Direct and indirect effects were calculated in three steps. First, the outcome variable, general knowledge of NSSI behaviors was entered as the dependent variable while role of school-based professional served as the predictor variable. Next, independent variable, role of schoolbased professional, continued as the predictor variable while mediating variable, direct experience with NSSI, was entered as the dependent variable. Finally, both the predictor and mediating variable was entered as predictor variables while general knowledge of NSSI behaviors was entered as the dependent variable.

The current study examined the role of school-based professional on outcome variables general knowledge of NSSI behaviors and perceived self-efficacy. Direct 
experience with mental health disorders and familiarity with mental health disorders were mediators between role of school based professional and outcome variables. It was hypothesized both mediators indirectly and significantly predict professional's general knowledge of NSSI behaviors and their perceived self-efficacy based upon their role in school. 
Regression and Path Analysis Research Question \#2A: Is the direct effect significantly greater than the indirect effect between role of school-based professional and their perceived self-efficacy as mediated by direct experience with NSSI behaviors?

Table 22 provides a detailed account of each model by school-based professional as it pertains to research question $2 \mathrm{~A}$. Respective models follow the table with direct and mediated path coefficients. 
Table 22

Summary of Mediation Analyses for Direct Experience with NSSI Behaviors and Professionals' Perceived Self-Efficacy Variables by Role of School-Based Professional

\begin{tabular}{lllllll}
\hline Variable & $\beta$ & $\mathrm{B}$ & $\mathrm{SE}$ & $\mathrm{p}<$ & $\mathrm{R}^{2}$ & $\mathrm{R}^{2} \Delta$ \\
\hline
\end{tabular}

Model 1: Predicting Professionals' Perceived Self-Efficacy

Step 1:

Role of School-Based Professional

$\begin{array}{rcccc}\text { School Social Worker } & .47 & 6.15 & .64 & .000^{* * *} \\ \text { Special Education Teacher } & -.17 & -2.88 & .94 & .002 * * \\ \text { Support Staff } & -.21 & -4.06 & 1.06 & .000^{* * *} \\ \text { School Nurse } & -.02 & -.35 & 1.04 & .733 \\ \text { School/District Administrator } & .02 & .57 & 1.43 & .690 \\ \text { Mental Health and Counseling Associate } & .18 & 3.88 & 1.18 & .001 * *\end{array}$

Step 2:

$.40 * * * .09 * * *$

Role of School-Based Professional

$\begin{array}{rllll}\text { School Social Worker } & .28 & 3.69 & .64 & .000^{* * *} \\ \text { Special Education Teacher } & -.10 & 2.54 & .22 & .039^{*} \\ \text { Support Staff } & -.12 & -2.29 & .91 & .013^{*} \\ \text { School Nurse } & -.04 & -.72 & .87 & .409 \\ \text { School/District Administrator } & -.02 & -.50 & 1.20 & .676 \\ \text { Mental Health and Counseling Associate } & .11 & 2.40 & 1.00 & .017^{*}\end{array}$

Direct Experience with NSSI Behaviors

$\begin{array}{rcccc}\text { School Social Worker } & .43 & 2.01 & .23 & .000^{* * *} \\ \text { Special Education Teacher } & .54 & 2.54 & .22 & .000^{* * *} \\ \text { Support Staff } & .53 & 2.50 & .22 & .000^{* * *} \\ \text { School Nurse } & .55 & 2.60 & .22 & .000^{* * *} \\ \text { School/District Administrator } & .55 & 2.60 & .22 & .000^{* * *} \\ \text { Mental Health and Counseling Associate } & .54 & 2.53 & .22 & .000^{* * *}\end{array}$

Model 2: Predicting Professionals' Direct Experience with NSSI Behaviors

Step 1:

$.28 * * *$

Role of School-Based Professional

$\begin{array}{lllll}\text { School Social Worker } & .44 & 1.23 & .14 & .000 * * *\end{array}$

Special Education Teacher $\begin{array}{lllll}-.13 & -.48 & .20 & .018^{*}\end{array}$

$\begin{array}{lllll}\text { Support Staff } & -.17 & -.71 & .23 & .002 * *\end{array}$

$\begin{array}{lllll}\text { School Nurse } & .04 & .14 & .22 & .514\end{array}$

$\begin{array}{lllll}\text { School/District Administrator } & .08 & .42 & .30 & .170\end{array}$

Mental Health and Counseling Associate $\begin{array}{lllll}.13 & .59 & .25 & .019^{*}\end{array}$

*Statistical significance: $* \mathrm{p}<.05, * * \mathrm{p}<.01, * * * \mathrm{p}<.001$ 
Figure 1

Path Analysis Model for Special Education Teachers' Perceived Self-Efficacy as Mediated by Direct Experience with NSSI Behaviors

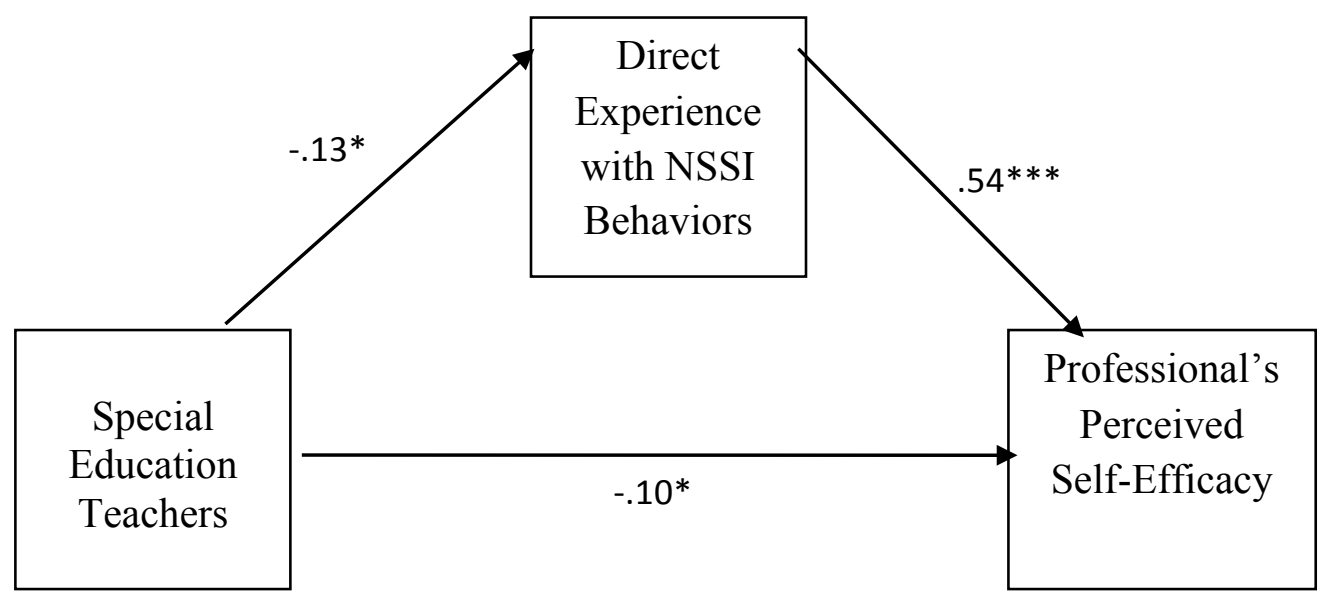

Bivariate Linear Regression and path analytic methods were employed to determine how well direct experience mediates the professional's role as a special education teacher and their perceived self-efficacy. Figure 1 represents a significant, partially mediated model. This suggests that although the relationship between professional's role as a special education teacher and their perceived self-efficacy is slightly diminished due to partial mediation by professional's direct experience with NSSI behaviors, it remains significant in the presence of the mediator. Indirect effect coefficients were calculated by taking the product of the coefficients from the predictor variable to the mediator and the mediator to the outcome variable. These values are presented in Table 22 for models related to research question $2 \mathrm{~A}$.

Sobel calculations were conducted to determine if the indirect effect is significant. The Sobel calculator is available through a reputable online website operated by Preacher (2013) at http://www.quantpsy.org/sobel/sobel.htm . The Sobel statistic indicates the 
indirect effect is significant $(Z=-2.33, p<.020)$. This suggest the mediating effect significantly intervenes and contributes to causal inferences of how school-based professional's direct experience with NSSI behaviors impacts their perceived selfefficacy while controlling for the school-based professional's role as a special education teacher.

Figure 2

Path Analysis Model for School Social Workers' Perceived Self-Efficacy as Mediated by Direct Experience with NSSI Behaviors

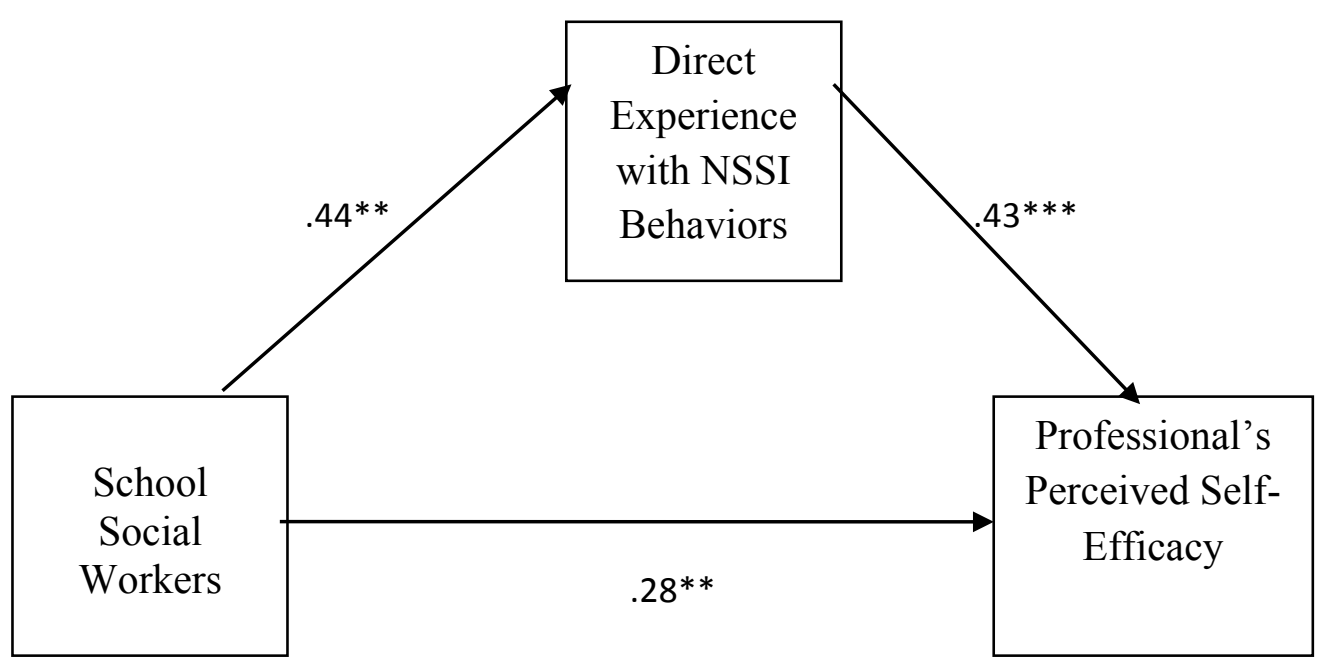

Figure 2 represents a significant, partially mediated model. This suggests that although the relationship between professional's role as a school social worker and their perceived self-efficacy is slightly diminished due to partial mediation by professional's direct experience with NSSI behaviors, it remains significant in the presence of the mediator. Sobel calculations were conducted to determine if the indirect effect is 
significant. The Sobel statistic indicates the indirect effect is significant $(\mathrm{Z}=6.23, \mathrm{p}<$ .000 ). This suggest the mediating effect significantly intervenes and contributes to causal inferences of how school-based professional's direct experience with NSSI behaviors impacts their perceived self-efficacy while controlling for the school-based professional's role as a school social worker.

Figure 3

Path Analysis Model for Support Staff's Perceived Self-Efficacy as Mediated by Direct Experience with NSSI Behaviors

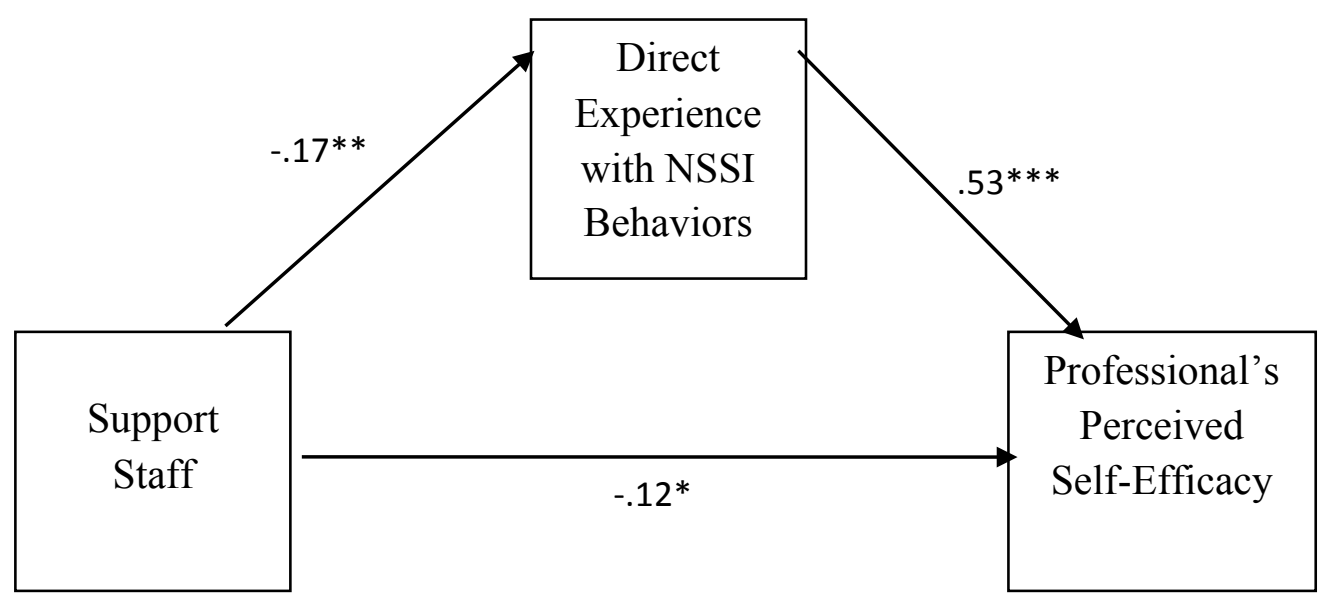

Figure 3 represents a significant, partially mediated model. This suggests that although the relationship between professional's role as support staff and their perceived self-efficacy is slightly diminished due to partial mediation by professional's direct experience with NSSI behaviors, it remains significant in the presence of the mediator. Sobel calculations were conducted to determine if the indirect effect was significant. The Sobel statistic indicates the indirect effect is significant $(Z=-3.00, p<.003)$. This suggest the mediating effect significantly intervenes and contributes to causal inferences 
of how school-based professional's direct experience with NSSI behaviors impacts their perceived self-efficacy while controlling for the school-based professional's role as support staff.

Figure 4

Path Analysis Model for School and District Administrators' Perceived Self-Efficacy as Mediated by Direct Experience with NSSI Behaviors

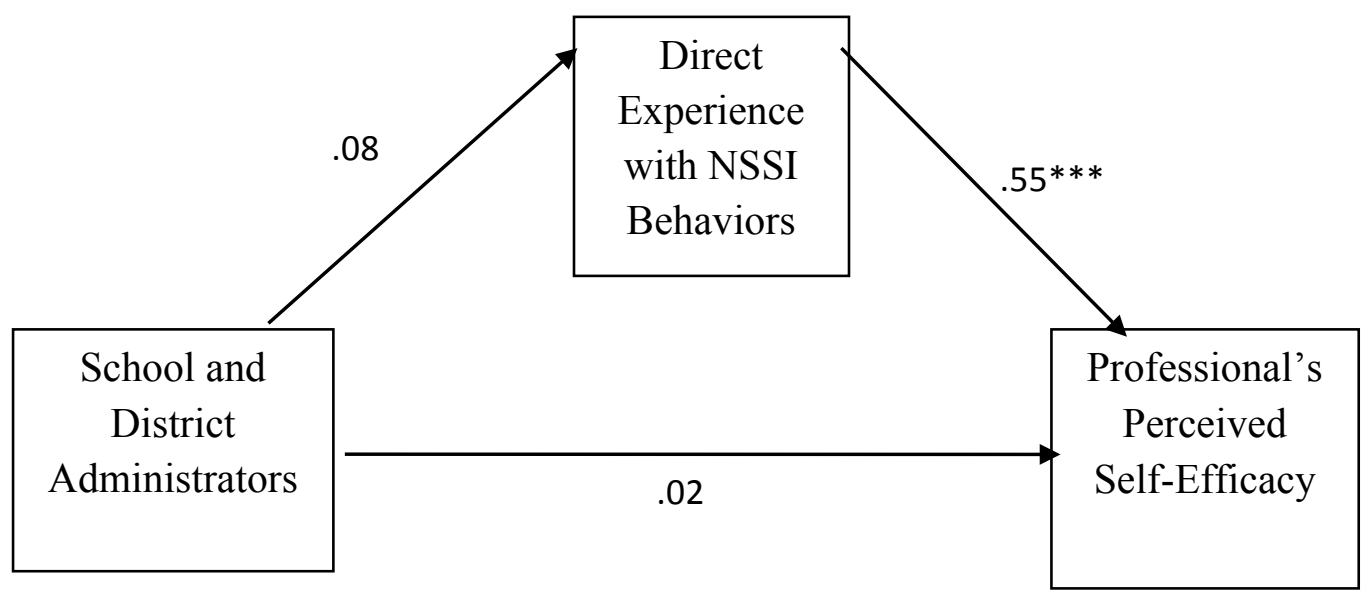

Figure 4 represents a nonsignificant, unmediated model. This suggests the relationship between the professional's role as a school or district administrator does not significantly predict their perceived self-efficacy. Additionally, having direct experience with NSSI behaviors does not significantly mediate the relationship between their role and perceived self-efficacy. Sobel calculations were conducted to determine the significance of indirect effects. The Sobel statistic indicates the indirect effect is not significant $(Z=1.37, p<.171)$. 
Figure 5

Path Analysis Model for School Nurses' Perceived Self-Efficacy as Mediated by Direct Experience with NSSI Behaviors

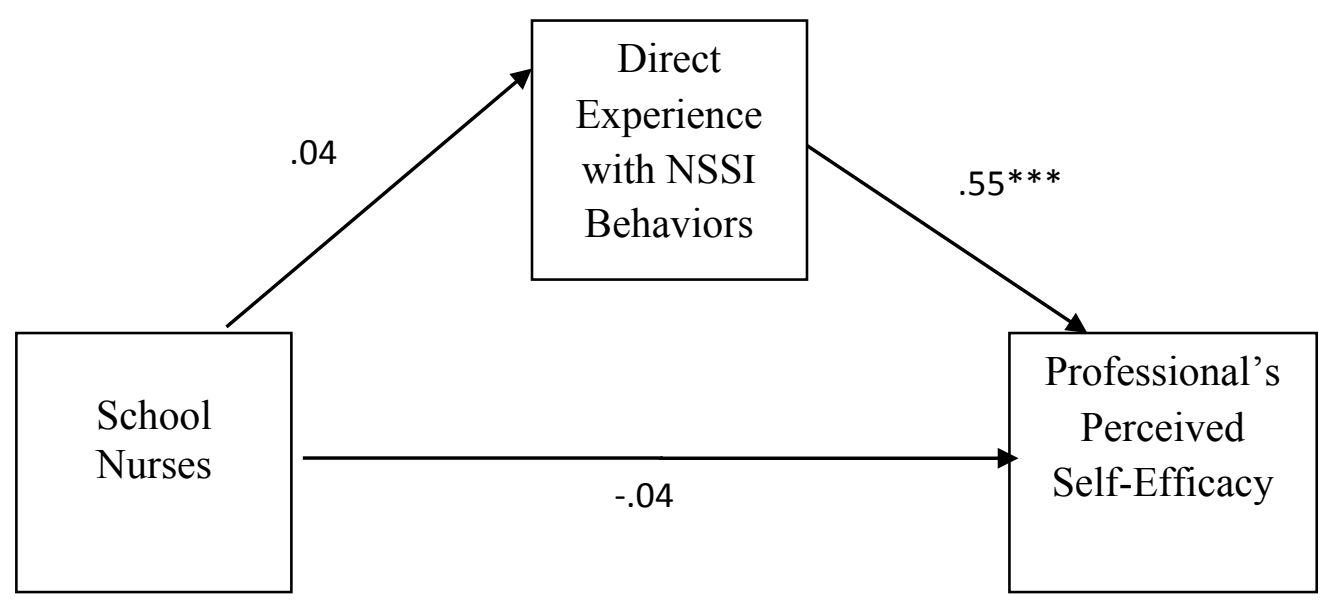

Figure 5 represents a nonsignificant, unmediated model. This suggests

professional's role as a school nurse does not significantly predict their perceived self-

efficacy. Additionally, having direct experience with NSSI behaviors does not significantly mediate the relationship between their role as a school nurse and perceived self-efficacy Sobel calculations were conducted to determine the significance of indirect effects. The Sobel statistic indicates the indirect effect is not significant $(Z=.65, p<$ $.516)$. 
Figure 6

Path Analysis Model for Mental Health and Counseling Associates' Perceived SelfEfficacy as Mediated by Direct Experience with NSSI Behaviors

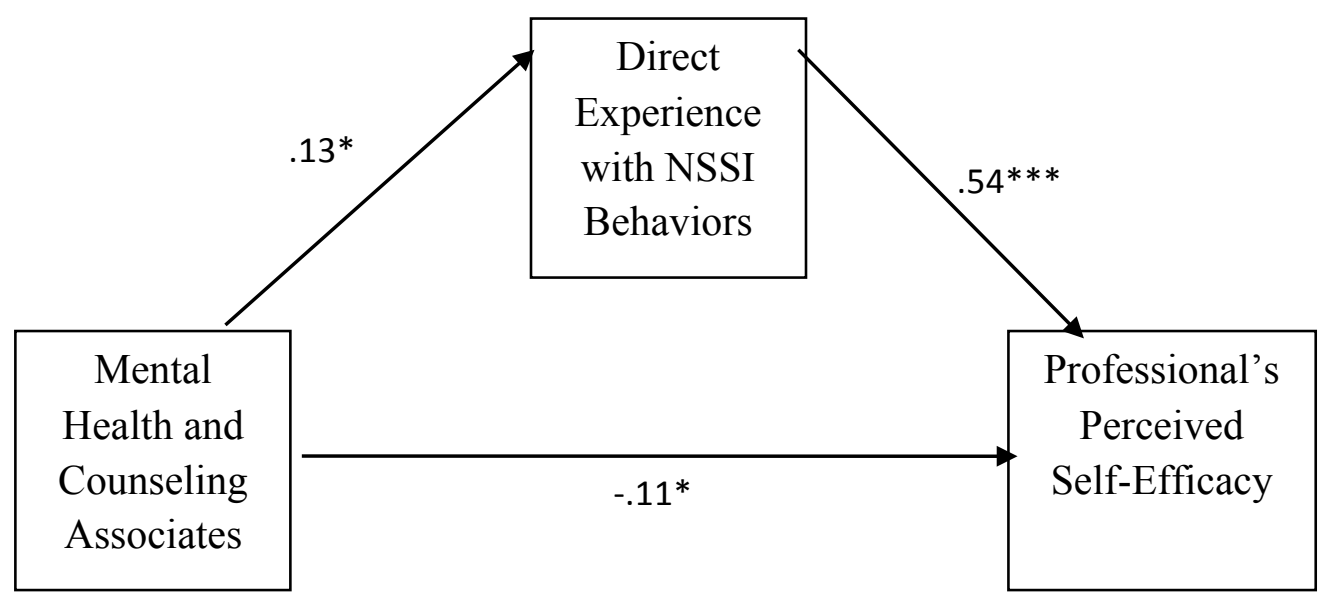

Figure 6 represents a significant, partially mediated model. This suggests that although the relationship between professional's role as a mental health and counseling associate and their perceived self-efficacy is slightly diminished due to partial mediation by professional's direct experience with NSSI behaviors, it remains significant in the presence of the mediator. Sobel calculations were conducted to determine if the indirect effect is significant. The Sobel statistic indicates the indirect effect is significant $(Z=$ $2.32, \mathrm{p}<.020)$. This suggest the mediating effect significantly intervenes and contributes to causal inferences of how school-based professional's direct experience with NSSI behaviors impacts their perceived self-efficacy while controlling for the school-based professional's role as a mental health or counseling associate. 
Regression and Path Analysis Research Question \#2B: Is the direct effect significantly greater than the indirect effect between role of school-based professional and their perceived self-efficacy as mediated by familiarity with various mental health disorders?

Table 23 provides a detailed account of each model by school-based professional as it pertains to research question $2 \mathrm{~B}$. Respective models follow the table with direct and mediated path coefficients. 
Table 23

Summary of Mediation Analyses for Familiarity with Mental Health Disorders Behaviors and Professionals' Perceived Self-Efficacy Variables by Role of School-Based Professional

\begin{tabular}{|c|c|c|c|c|c|c|}
\hline Variable & $\beta$ & $\mathrm{B}$ & SE & $\mathrm{p}<$ & $\mathrm{R}^{2}$ & $\mathrm{R}^{2} \Delta$ \\
\hline \multicolumn{7}{|c|}{ Model 1: Predicting Professionals' Perceived Self-Efficacy } \\
\hline \multicolumn{7}{|l|}{ Role of School-Based Professional } \\
\hline School Social Worker & .47 & 6.15 & .64 & $.000 * * *$ & & \\
\hline Special Education Teacher & -.17 & -2.88 & .94 & $.002 * *$ & & \\
\hline Support Staff & -.21 & -4.06 & 1.06 & $.000 * * *$ & & \\
\hline School Nurse & -.02 & -.35 & 1.04 & .733 & & \\
\hline School/District Administrator & .02 & .57 & 1.43 & .690 & & \\
\hline $\begin{array}{l}\text { Mental Health and Counseling Associate } \\
\text { Step 2: }\end{array}$ & .18 & 3.88 & 1.18 & $.001 * *$ & & \\
\hline Role of School-Based Professional & & & & & $.36 * * *$ & $.05 * * *$ \\
\hline School Social Worker & .39 & 5.13 & .62 & $.000 * * *$ & & \\
\hline Special Education Teacher & -.15 & -2.68 & .86 & $.002 * *$ & & \\
\hline Support Staff & -.12 & -2.31 & 1.01 & $.023 *$ & & \\
\hline School Nurse & -.08 & -1.41 & .95 & .138 & & \\
\hline School/District Administrator & -.01 & -.29 & 1.31 & .823 & & \\
\hline Mental Health and Counseling Associate & .12 & 2.59 & 1.09 & $.018 *$ & & \\
\hline \multicolumn{7}{|l|}{$\begin{array}{c}\text { Familiarity with Mental } \\
\text { Health Disorders }\end{array}$} \\
\hline School Social Worker & .32 & .68 & .10 & $.000 * * *$ & & \\
\hline Special Education Teacher & .41 & .87 & .11 & $.000 * * *$ & & \\
\hline Support Staff & .39 & .83 & .11 & $.023 *$ & & \\
\hline School Nurse & .42 & .90 & .11 & $.000 * * *$ & & \\
\hline School/District Administrator & .42 & .88 & .11 & $.000 * * *$ & & \\
\hline Mental Health and Counseling Associate & .40 & .84 & .11 & $.000 * * *$ & & \\
\hline
\end{tabular}

Model 2: Predicting Professionals' Familiarity with Mental Health Disorders

Step 1:

Role of School-Based Professional

School Social Worker $\begin{array}{llll}.24 & 1.49 & .33 & .000^{* * *}\end{array}$

$\begin{array}{lllll}\text { Special Education Teacher } & -.03 & -.23 & .45 & .609\end{array}$

$\begin{array}{lllll}\text { Support Staff } & -.23 & -2.13 & .50 & .000 * * *\end{array}$

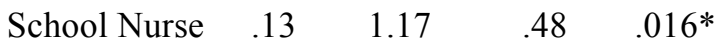

$\begin{array}{lllll}\text { School/District Administrator } & .08 & .98 & .67 & .146\end{array}$

$\begin{array}{lllll}\text { Mental Health and Counseling Associate } & .15 & 1.54 & .56 & .006^{* *}\end{array}$

*Statistical significance: ${ }^{*} \mathrm{p}<.05,{ }^{* *} \mathrm{p}<.01,{ }^{* * *} \mathrm{p}<.001$ 
Figure 7

Path Analysis Model for Mental Health and Counseling Associates' Perceived SelfEfficacy as Mediated by Familiarity with Various Mental Health Disorders

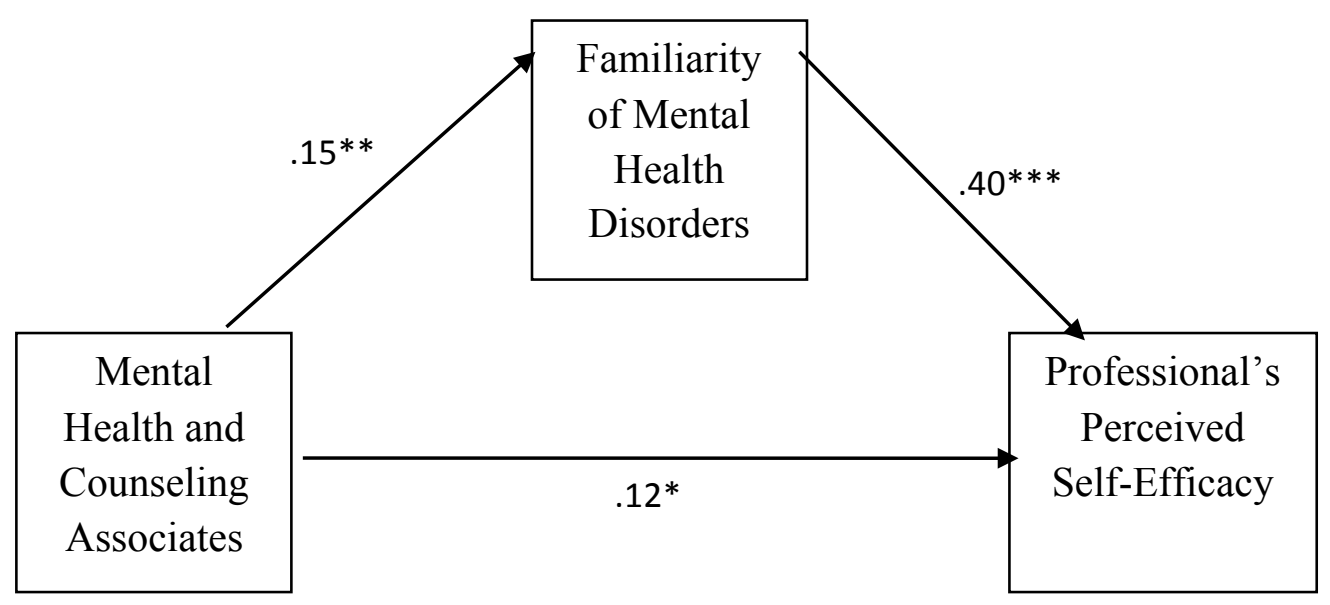

Figure 7 represents a significant, partially mediated model. This suggests that the relationship between the professional's role as a mental health and counseling associate and their perceived self-efficacy is only slightly diminished due to the partial mediation by professional's familiarity with various mental health disorders. However, the relationship remains significant in the presence of the mediator. Sobel calculations were conducted to determine if the indirect effect is significant. The Sobel statistic indicates the indirect effect is significant $(Z=2.61, \mathrm{p}<.009)$. This suggests familiarity with various mental health disorders significantly mediates the relationship and accounts for variance in professional's perceived self-efficacy while controlling for their role as a mental health and counseling associates. 
Figure 8

Path Analysis Model for School Social Workers' Perceived Self-Efficacy as Mediated by Familiarity with Various Mental Health Disorders

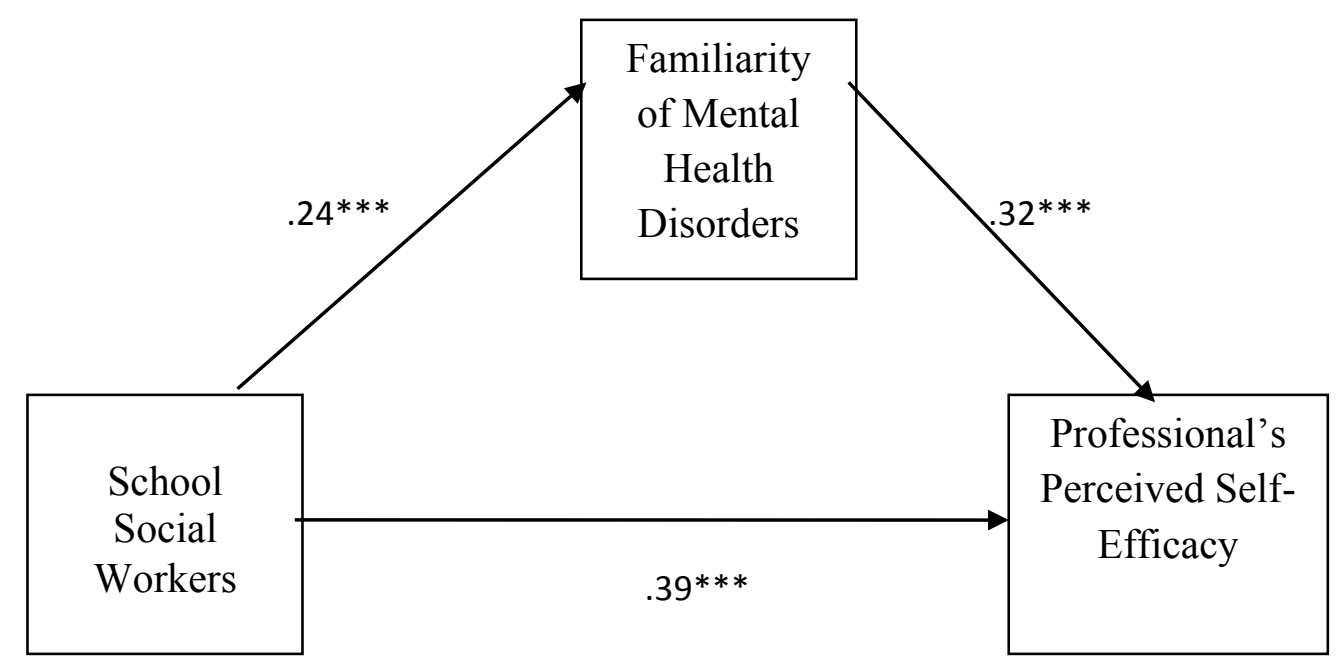

Figure 8 represents a significant, partially mediated model. This suggests that although the relationship between the professional's role as a social worker and their perceived self-efficacy is slightly diminished due to the partial mediation by professional's familiarity with various mental health disorders, it remains significant in the presence of the mediator. This scenario is also interesting given that the direct effect is greater than the indirect effect. This suggest that the mediated relationship is significant in its own right; however, it does not significantly add any more explanatory power to the prediction of school social worker's perceived self-efficacy scores. Sobel calculations were conducted to determine if the indirect effect is significant. The Sobel statistic indicates the indirect effect is significant $(Z=3.77, p<.000)$. This suggests that familiarity with various mental health disorders significantly mediates the relationship 
and accounts for variance in professionals' perceived self-efficacy while controlling for their role as a school social worker.

Figure 9

Path Analysis Model for Support Staff's Perceived Self-Efficacy as Mediated by Familiarity with Various Mental Health Disorders

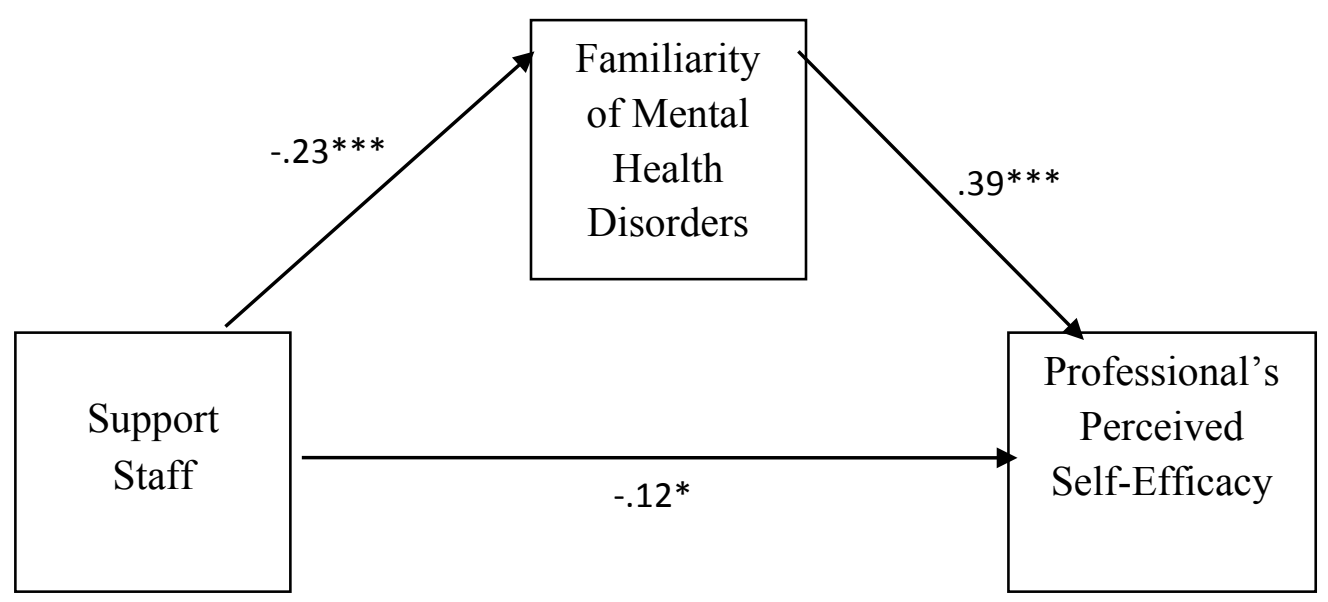

Figure 9 represents a significant, partially mediated model. This suggests that although the relationship between the professional's role as support staff and their perceived self-efficacy is slightly diminished due to the partial mediation by professional's familiarity with various mental health disorders, it remains significant in the presence of the mediator. Sobel calculations were conducted to determine if the indirect effect is significant. The Sobel statistic indicates the indirect effect is significant $(\mathrm{Z}=-3.73, \mathrm{p}<.000)$. This suggests that familiarity with various mental health disorders significantly mediates the relationship and accounts for variance in professional's perceived self-efficacy while controlling for their role as support staff. 
Figure 10

Path Analysis Model for School and District Administrators' Perceived Self-Efficacy as Mediated by Familiarity with Various Mental Health Disorders

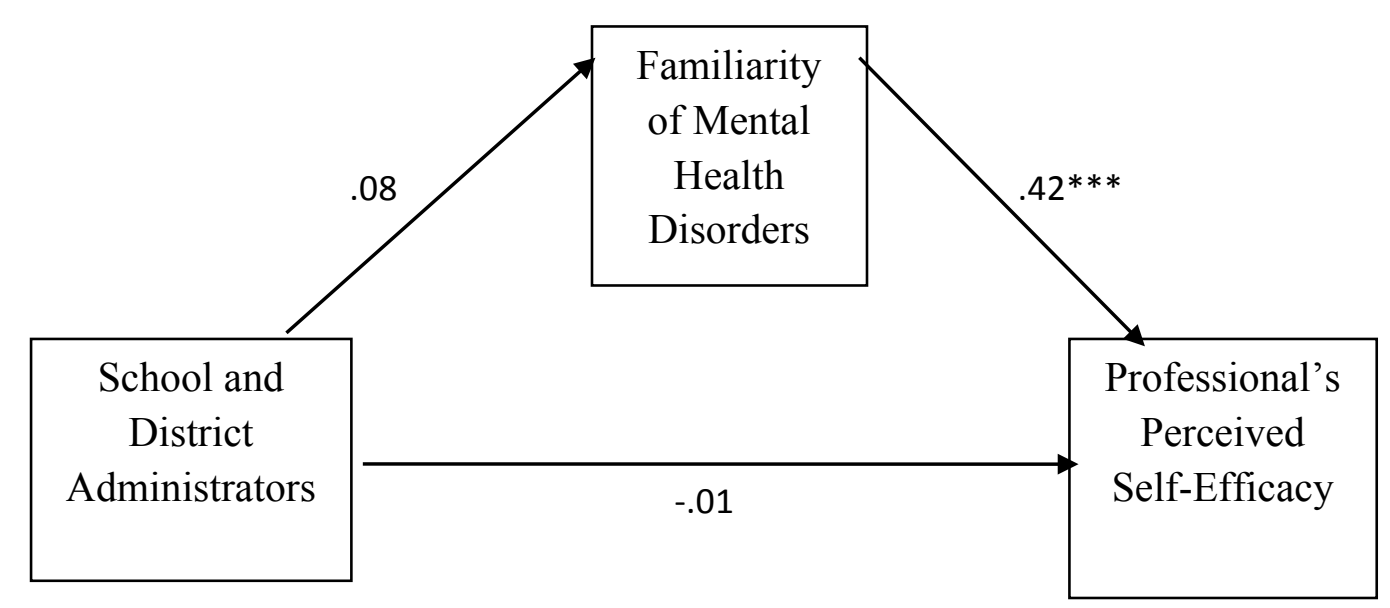

Figure 10 represents a nonsignificant, unmediated model. This suggests the relationship between professional's role as a school or district administrator does not significantly predict their perceived self-efficacy. Additionally, familiarity with various mental health disorders does not significantly mediate the relationship between their role and their perceived self-efficacy. Sobel calculations were conducted to determine if the indirect effect is nonsignificant as well. The Sobel statistic indicates the indirect effect is not significant $(\mathrm{Z}=1.44, \mathrm{p}<.151)$. 
Figure 11

Path Analysis Model for School Nurses' Perceived Self-Efficacy as Mediated by Familiarity with Various Mental Health Disorders

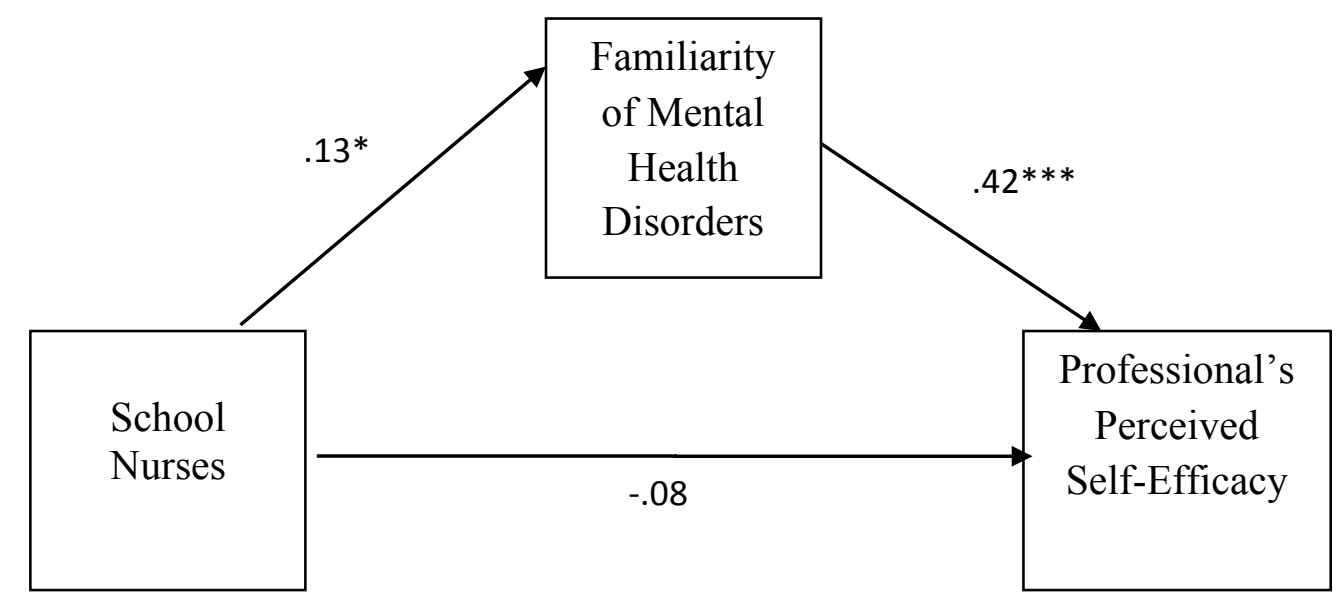

Figure 11 represents a significant, fully mediated model. This suggests the relationship between professional's role as a school nurse and their perceived selfefficacy is significantly diminished due to the mediation by professional's familiarity with various mental health disorders and is no longer significant in the presence of the mediator. Sobel calculations were conducted to determine if the indirect effect was significant. The Sobel statistic indicates the indirect effect is significant $(Z=2.33, p<$ .020). This suggests that familiarity with various mental health disorders significantly mediates the relationship and accounts for variance in professional's perceived selfefficacy while controlling for their role as a school nurse. 
Figure 12

Path Analysis Model for Special Education Teachers' Perceived Self-Efficacy as Mediated by Familiarity with Various Mental Health Disorders

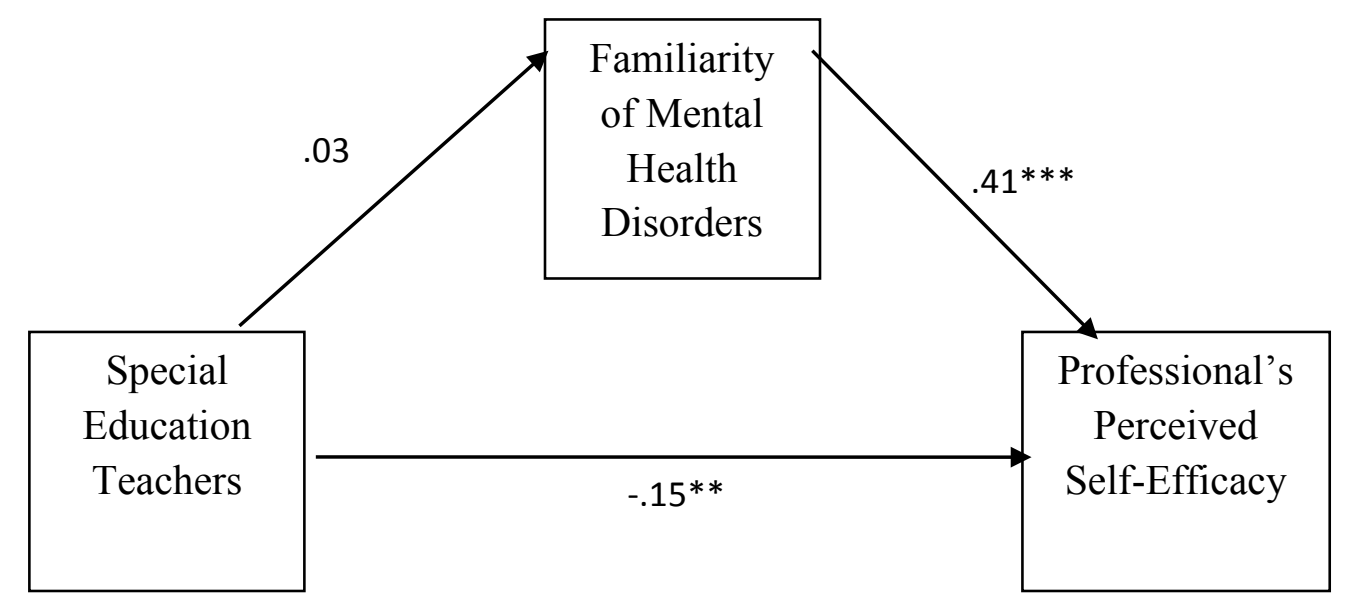

Figure 12 represents a nonsignificant, unmediated model. This suggests the relationship between professional's role as a mental health and counseling associate and their perceived self-efficacy is not significantly mediated by professionals' familiarity with various mental health disorders. Sobel calculations were conducted to determine if the indirect effect is significant. The Sobel statistic indicates the indirect effect is not significant $(Z=-.51, \mathrm{p}<.609)$. 
Path Analysis Research Question \#3A: Is the direct effect significantly greater than the indirect effect between role of school-based professional and general knowledge of NSSI behaviors as mediated by direct experience with NSSI behaviors?

Table 24 provides a detailed account of each model by school-based professional as it pertains to research question 3A. Respective models follow the table with direct and mediated path coefficients. 
Table 24

Summary of Mediation Analyses for Direct Experience with NSSI Behaviors and Professionals' General Knowledge of NSSI Behaviors Variables by Role of SchoolBased Professional

\begin{tabular}{|c|c|c|c|c|}
\hline Variable & $\beta$ & B & SE & $\mathrm{p}<$ \\
\hline
\end{tabular}

Step 1:

Model 1: Predicting Professionals' General Knowledge of NSSI Behaviors

Role of School-Based Professional

$\begin{array}{rcccc}\text { School Social Worker } & .17 & 1.98 & .62 & .002^{* *} \\ \text { Special Education Teacher } & -.07 & -1.08 & .84 & .197 \\ \text { Support Staff } & -.03 & -.57 & .95 & .551 \\ \text { School Nurse } & -.00 & -.07 & .90 & .936 \\ \text { School/District Administrator } & -.17 & -3.88 & 1.24 & .002^{* *} \\ \text { Mental Health and Counseling Associate } & .03 & .55 & 1.05 & .601\end{array}$

Step 2:

$.06 * * \quad .00$

Role of School-Based Professional

$\begin{array}{rllll}\text { School Social Worker } & .14 & 1.62 & .70 & .020^{*} \\ \text { Special Education Teacher } & -.06 & -.84 & .84 & .318 \\ \text { Support Staff } & -.01 & -.20 & .96 & .839 \\ \text { School Nurse } & -.01 & -.15 & .90 & .865 \\ \text { School/District Administrator } & -.18 & -4.14 & 1.23 & .001^{* *} \\ \text { Mental Health and Counseling Associate } & .01 & .23 & 1.05 & .828\end{array}$

Direct Experience with NSSI Behaviors

$\begin{array}{rcccl}\text { School Social Worker } & .07 & .28 & .25 & .260 \\ \text { Special Education Teacher } & .12 & .51 & .23 & .025^{*} \\ \text { Support Staff } & .13 & .53 & .23 & .021^{*} \\ \text { School Nurse } & .13 & .54 & .23 & .017^{*} \\ \text { School/District Administrator } & .15 & .60 & .22 & .008^{* *} \\ \text { Mental Health and Counseling Associate } & .13 & .54 & .23 & .020^{*}\end{array}$

Model 2: Predicting Professionals' Direct Experience with NSSI Behaviors

Step 1:

Role of School-Based Professional

School Social Worker Special Education Teache

Support Staff

School Nurse .04

$.44 \quad 1.23$

$.23 \quad .14$

.14

$.000^{* * *}$

$-71$

Administrator .08

.14

.23

.22

$002 * *$

School/District Administrator

.42

.30

.514

Mental Health and Counseling Associate $\quad .13 \quad .59$

*Statistical significance: ${ }^{*} \mathrm{p}<.05,{ }^{* *} \mathrm{p}<.01,{ }^{* * *} \mathrm{p}<.001$ 
Figure 13

Path Analysis Model for Mental Health and Counseling Associates' General Knowledge of NSSI Behaviors as Mediated by Direct Experience with NSSI Behaviors

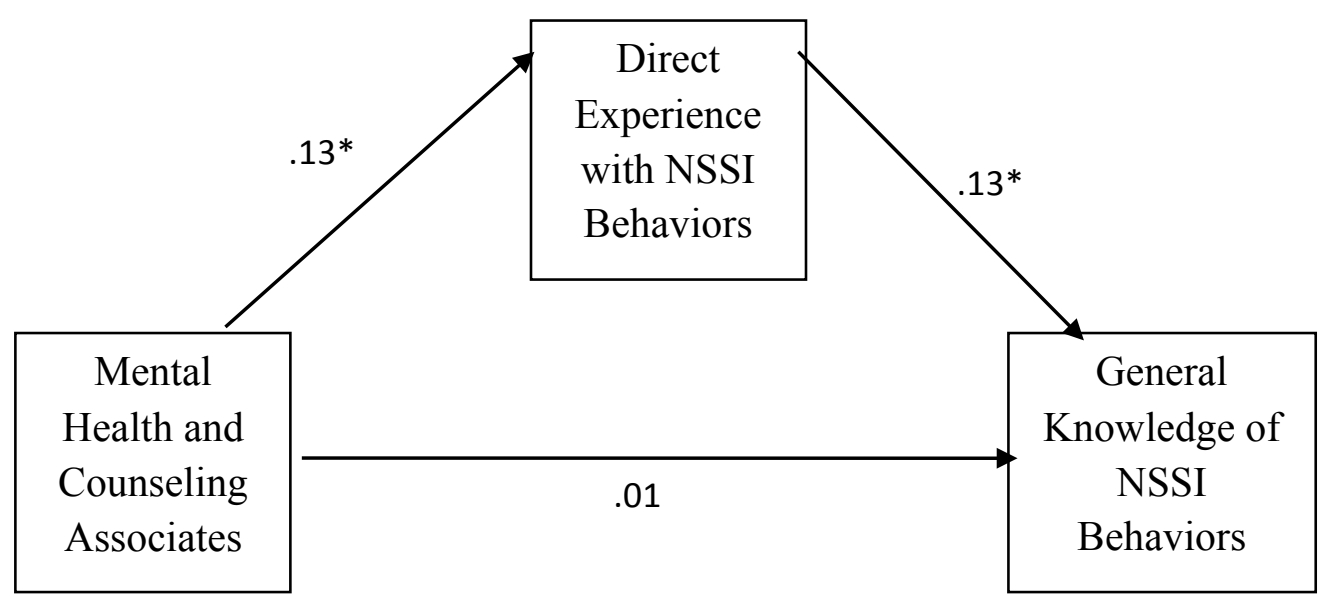

Figure 13 represents a nonsignificant, mediated model. This suggests the relationship between professional's role as a mental health and counseling associate and their general knowledge of NSSI behaviors is significantly diminished due to mediation by professional's direct experience with NSSI behaviors and is no longer significant in the presence of the mediator. Sobel calculations were conducted to determine if the indirect effect is significant. The Sobel statistic indicates the indirect effect is not significant $(Z=1.67, \mathrm{p}<.095)$. 
Figure 14

Path Analysis Model for School Social Workers' General Knowledge of NSSI Behaviors as Mediated by Direct Experience with NSSI Behaviors

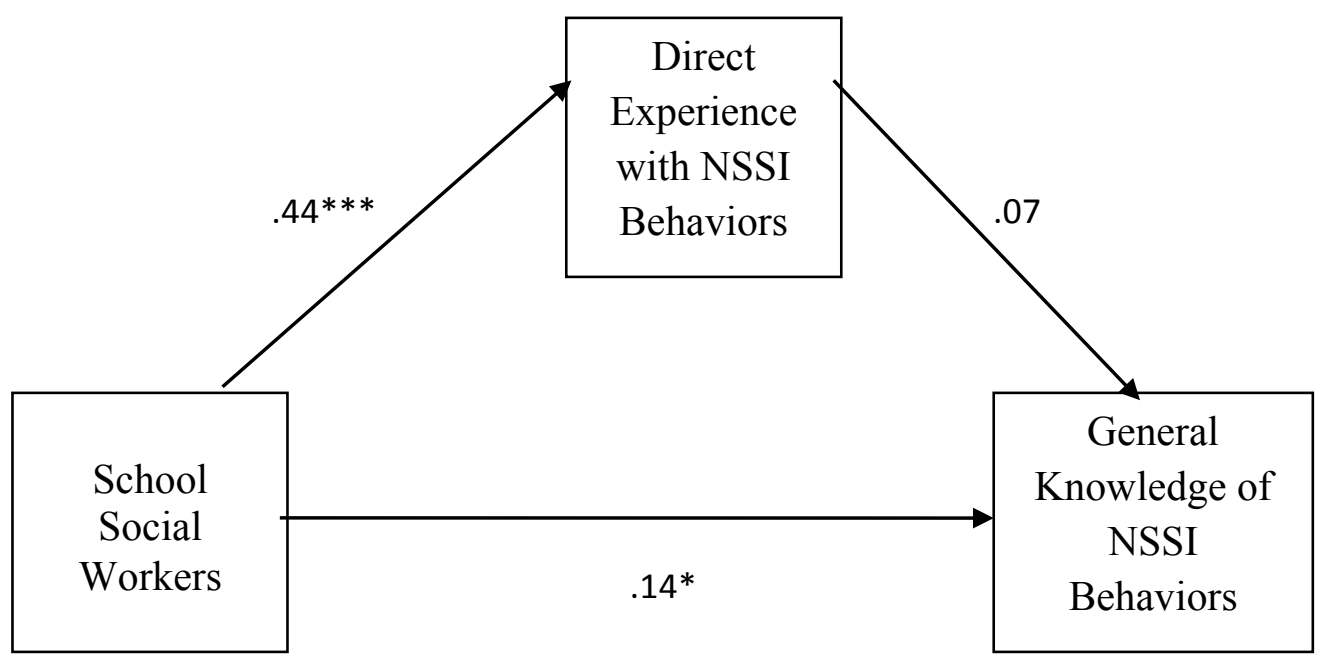

Figure 14 represents a nonsignificant, unmediated model. This suggests the relationship between professional's role as a social worker and their general knowledge of NSSI behaviors is not significantly mediated by professional's direct experience with NSSI behaviors. Sobel calculations were conducted to determine if the indirect effect is significant. The Sobel statistic indicates the indirect effect is not significant $(Z=1.12, p<$ $.263)$. 
Figure 15

Path Analysis Model for Support Staff's General Knowledge of NSSI Behaviors as Mediated by Direct Experience with NSSI Behaviors

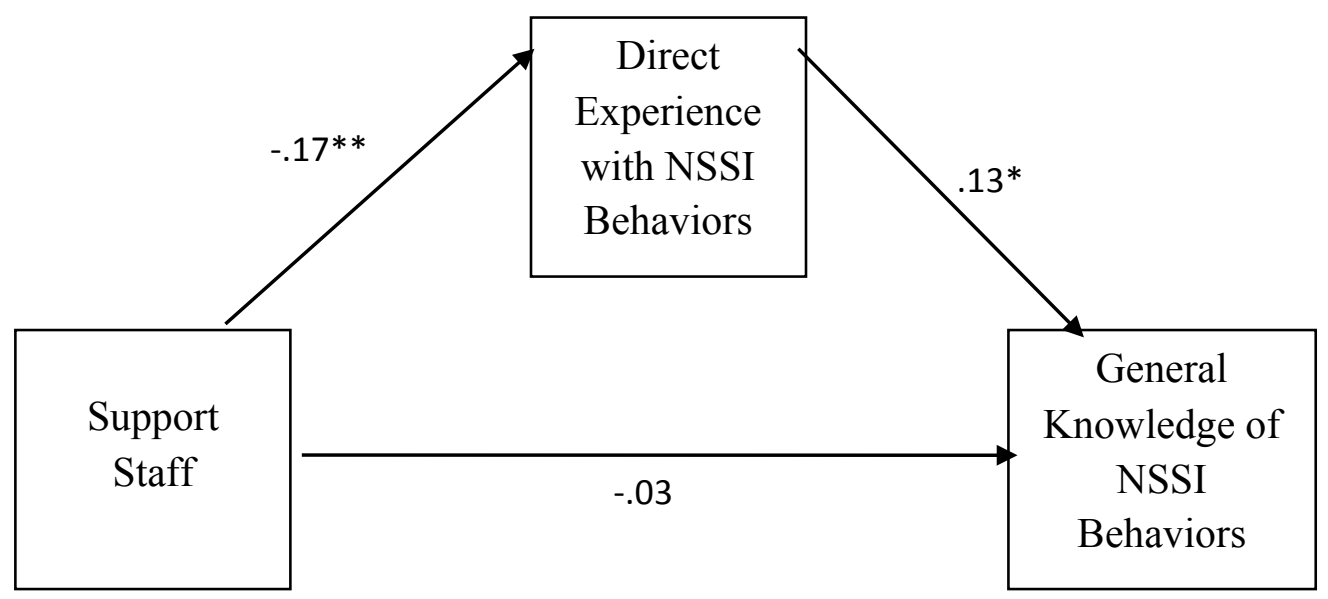

Figure 15 represents a nonsignificant, fully mediated model. This suggests the relationship between professional's role as support staff and their general knowledge of NSSI behaviors is significantly diminished due to mediation by professional's direct experience with NSSI behaviors. Sobel calculations were conducted to determine if the indirect effect is significant. The Sobel statistic indicates the indirect effect is significant $(Z=-1.86, p<.063)$. This suggests that although the mediator diminishes the direct effect, it does not significantly mediate the relationship between role of professional as support staff and their general knowledge of NSSI behaviors to the extent causal inferences are plausible. 
Figure 16

Path Analysis Model for School and District Administrators' General Knowledge of NSSI Behaviors as Mediated by Direct Experience with NSSI Behaviors

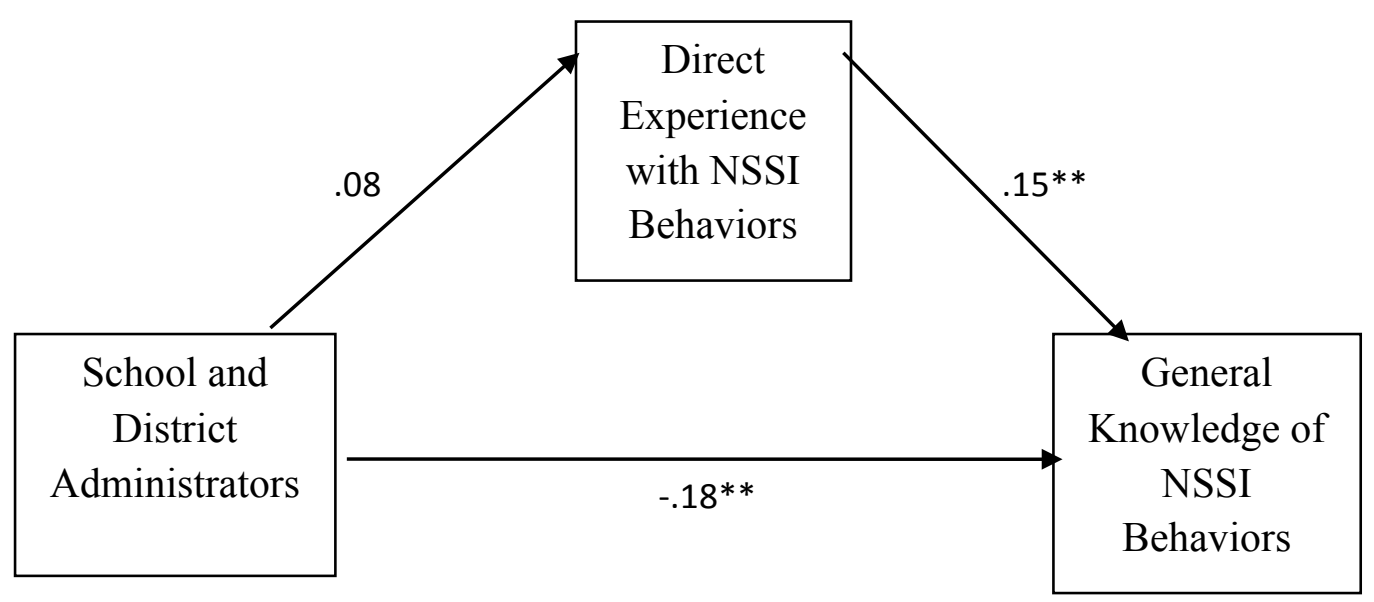

Figure 16 represents a nonsignificant, unmediated model. This suggests the relationship between professional's role as a school or district administrator and their general knowledge of NSSI behaviors is not diminished by mediation of professional's direct experience with NSSI behaviors and remains significant in the presence of the mediator. Sobel calculations were conducted to determine if the indirect effect is significant. The Sobel statistic indicates the indirect effect is not significant $(Z=1.23, p<$ $.220)$. 
Figure 17

Path Analysis Model for School Nurses' General Knowledge of NSSI Behaviors as Mediated by Direct Experience with NSSI Behaviors

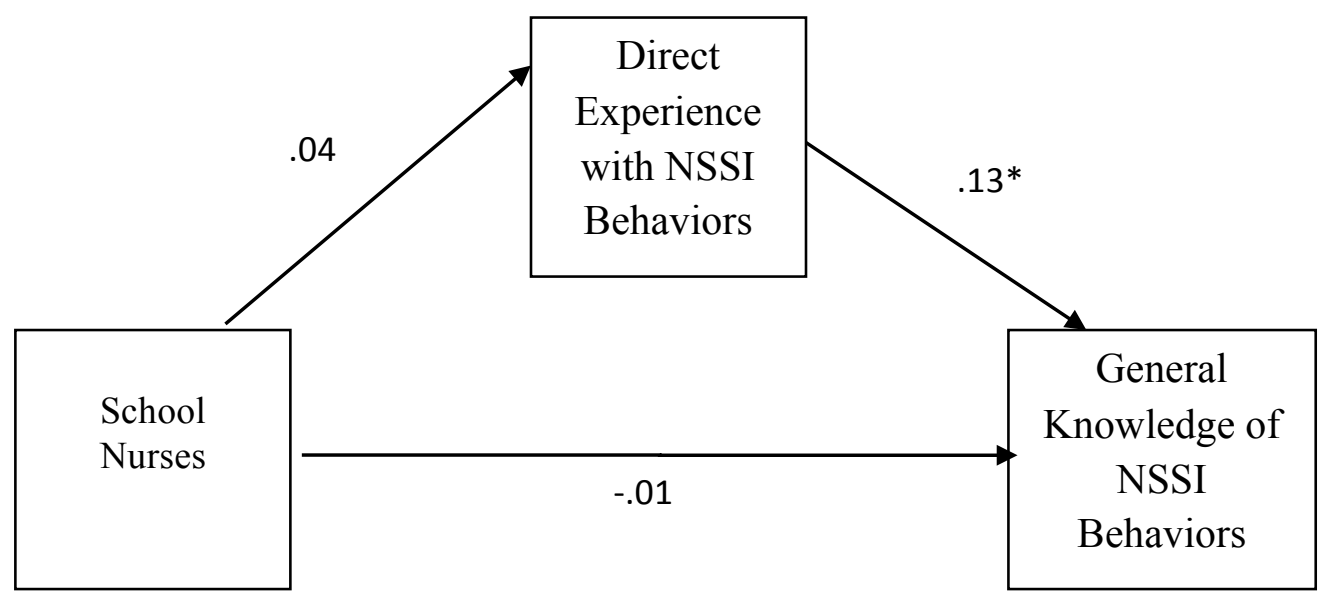

Figure 17 represents a nonsignificant, unmediated model. This suggests the relationship between professional's role as a school nurse and their general knowledge of NSSI behaviors is not significant in the presence of the mediator nor is the mediated relationship via direct experience with NSSI behaviors. Sobel calculations were conducted to determine if the indirect effect is significant. The Sobel statistic indicates the indirect effect is not significant $(\mathrm{Z}=.63, \mathrm{p}<.530)$. 
Figure 18

Path Analysis Model for Special Education Teachers' General Knowledge of NSSI Behaviors as Mediated by Direct Experience with NSSI Behaviors

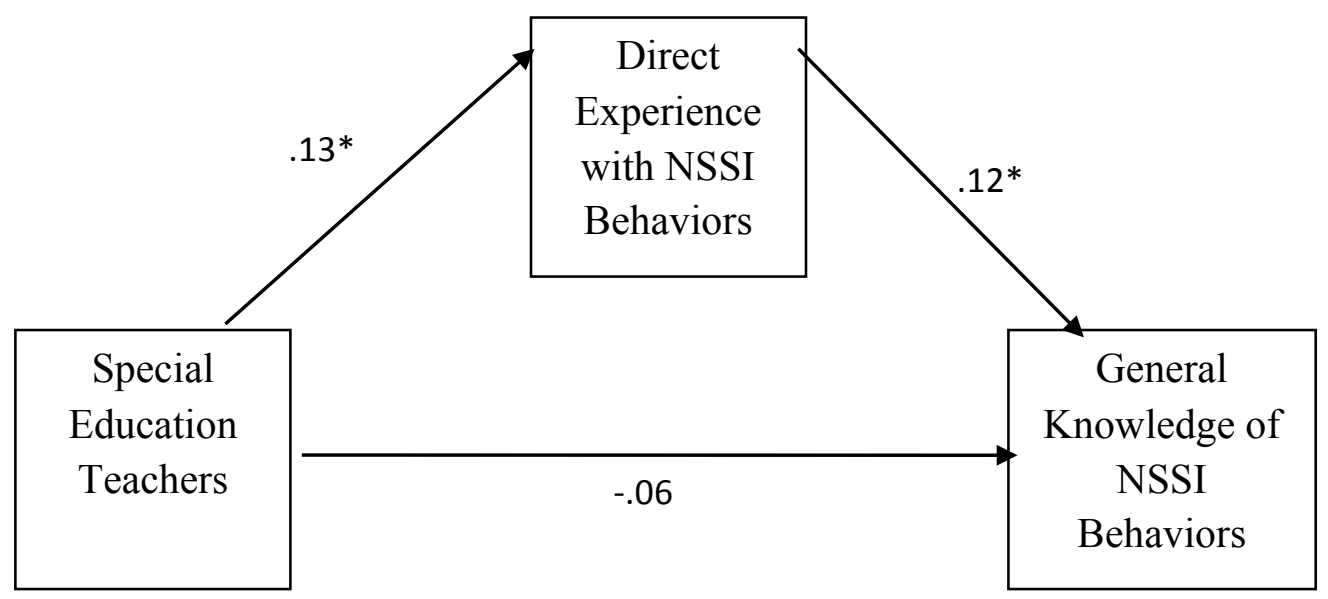

Figure 18 represents a nonsignificant, mediated model. This suggests the relationship between professional's role as a mental health and counseling associate and their general knowledge of NSSI behaviors is significantly diminished due to mediation by professional's Sobel calculations were conducted to determine if the indirect effect is significant. The Sobel statistic indicates the indirect effect is significant $(Z=-1.63, p<$ $.102)$. 
Regression and Path Analysis Research Question \#3B: Is the direct effect significantly greater than the indirect effect between role of school-based professional and general knowledge of NSSI behaviors as mediated by familiarity with various mental health disorders?

Table 25 provides a detailed account of each model by school-based professional as it pertains to research question 3B. Respective models follow the table with direct and mediated path coefficients. 
Table 25

Summary of Mediation Analyses for Familiarity with Mental Health Disorders and Professionals' General Knowledge of NSSI Behaviors Variables by Role of School-Based Professional

$\begin{array}{lllllll}\text { Variable } & \beta & \mathrm{B} & \mathrm{SE} & \mathrm{p}< & \mathrm{R}^{2} & \mathrm{R}^{2} \Delta\end{array}$

Model 1: Predicting Professionals' General Knowledge of NSSI Behaviors

Step 1:

$.06 * *$

Role of School-Based Professional

$\begin{array}{rcccc}\text { School Social Worker } & .17 & 1.98 & .62 & .002^{* *} \\ \text { Special Education Teacher } & -.07 & -1.08 & .84 & .197 \\ \text { Support Staff } & -.03 & -.57 & .95 & .551 \\ \text { School Nurse } & -.00 & -.07 & .90 & .936 \\ \text { School/District Administrator } & -.17 & -3.88 & 1.24 & .002^{* *} \\ \text { alth and Counseling Associate } & .03 & .55 & 1.05 & .601\end{array}$

Mental Health and Counseling Associate $\quad .03 \quad \begin{array}{llll}.55 & 1.05 & .601\end{array}$

Step 2:

Role of School-Based Professional

$\begin{array}{rllll}\text { School Social Worker } & .15 & 1.71 & .64 & .008^{* *} \\ \text { Special Education Teacher } & -.07 & -1.03 & .83 & .218 \\ \text { Support Staff } & -.00 & -.06 & .97 & .953 \\ \text { School Nurse } & -.02 & -.36 & .91 & .689 \\ \text { School/District Administrator } & -.18 & -4.15 & 1.23 & .001^{* *} \\ \text { th and Counseling Associate } & .01 & .18 & 1.05 & .864\end{array}$

$\begin{array}{llllll}\text { Mental Health and Counseling Associate } & .01 & .18 & 1.05 & .864\end{array}$

Familiarity with Mental

Health Disorders

$\begin{array}{rcccl}\text { School Social Worker } & .09 & .18 & .10 & .093 \\ \text { Special Education Teacher } & .13 & .24 & .10 & .019^{*} \\ \text { Support Staff } & .13 & .24 & .11 & .022^{*} \\ \text { School Nurse } & .13 & .25 & .10 & .016^{*} \\ \text { School/District Administrator } & .14 & .27 & .10 & .008^{* *} \\ \text { Salth and Counseling Associate } & .13 & .24 & .10 & .021^{*}\end{array}$

Model 2: Predicting Professionals' Familiarity with Mental Health Disorders

Step 1:

Role of School-Based Professional

$\begin{array}{rllll}\text { School Social Worker } & .24 & 1.49 & .33 & .000^{* * *} \\ \text { Special Education Teacher } & -.03 & -.23 & .45 & .609 \\ \text { Support Staff } & -.23 & -2.13 & .50 & .000^{* * *} \\ \text { School Nurse } & .13 & 1.17 & .48 & .016^{*} \\ \text { School/District Administrator } & .08 & .98 & .67 & .146 \\ \text { th and Counseling Associate } & .15 & 1.54 & .56 & .006^{* *}\end{array}$

Mental Health and Counseling Associate $\quad .15 \quad 1.54 \quad .56$

*Statistical significance: ${ }^{*} \mathrm{p}<.05,{ }^{* *} \mathrm{p}<.01,{ }^{* * *} \mathrm{p}<.001$ 
Figure 19

Path Analysis Model for Mental Health and Counseling Associates' General Knowledge of NSSI Behaviors as Mediated by Familiarity with Various Mental Health Disorders

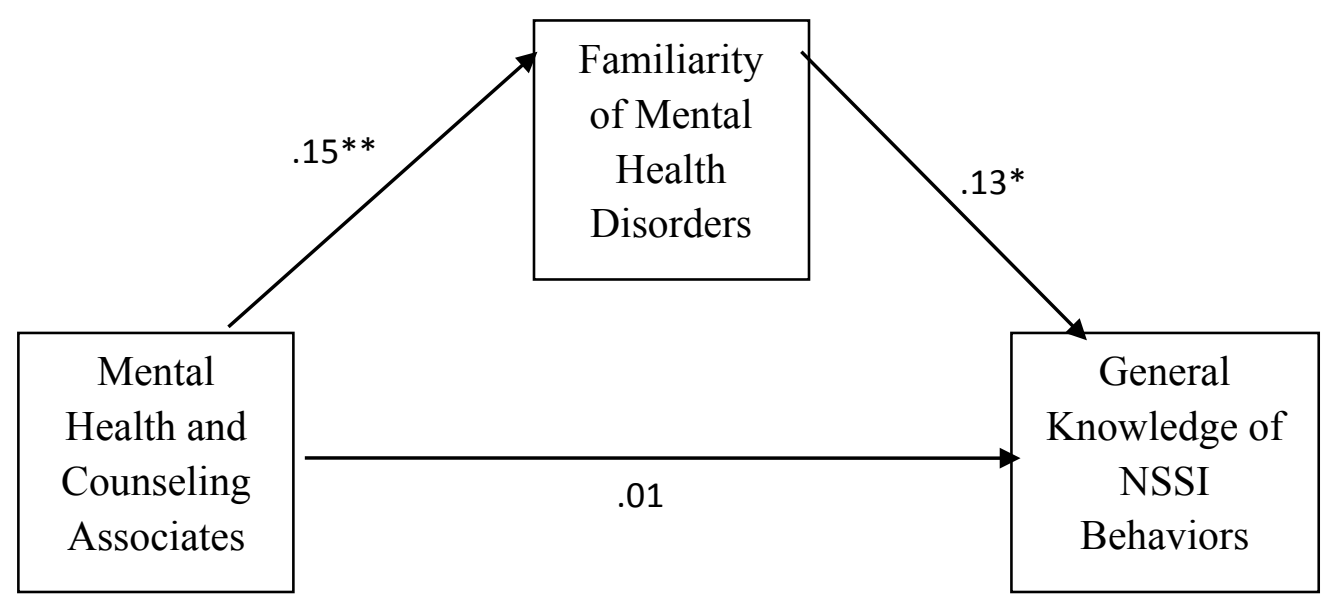

Figure 19 represents a significant, fully mediated model. This suggests the relationship between professional's role as a mental health and counseling associate and their general knowledge of NSSI behaviors is significantly diminished due to mediation by professional's familiarity with various mental health disorders. Sobel calculations were conducted to determine if the indirect effect is significant. The Sobel statistic indicates the indirect effect is not significant $(Z=1.78, \mathrm{p}<.075)$. This suggests that although the mediator diminishes the direct effect, it does not significantly mediate the relationship between role of professional as mental health and counseling associates and their general knowledge of NSSI behaviors to the extent causal inferences are plausible. 
Figure 20

Path Analysis Model for School Social Workers' General Knowledge of NSSI Behaviors as Mediated by Familiarity with Various Mental Health Disorders

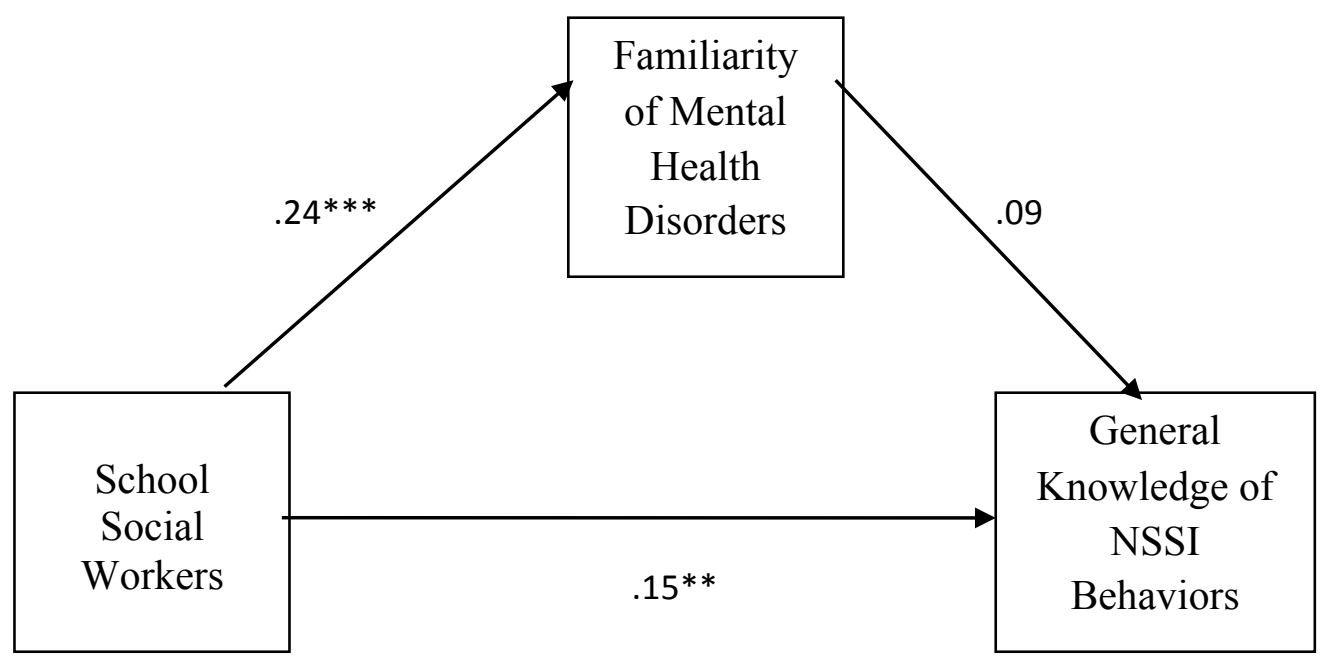

Figure 20 represents a nonsignificant, unmediated model. This suggests the relationship between professional's role as a social worker and their general knowledge of NSSI behaviors is not significantly diminished due to mediation by professional's familiarity with various mental health disorders. The direct effect remains significant in the presence of the mediator. Sobel calculations were conducted to determine if the indirect effect is significant. The Sobel statistic indicates the indirect effect is not significant $(\mathrm{Z}=1.58, \mathrm{p}<.115)$. 
Figure 21

Path Analysis Model for Support Staff's General Knowledge of NSSI Behaviors as Mediated by Familiarity with Various Mental Health Disorders

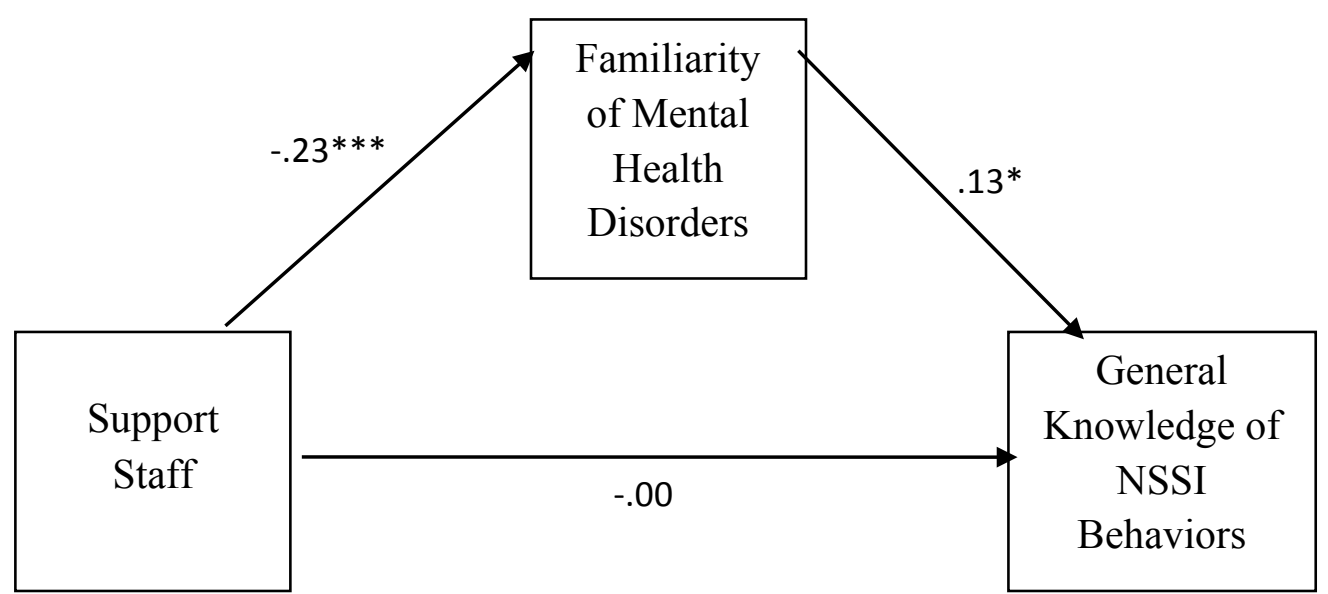

Figure 21 represents a significant, fully mediated model. This suggests the relationship between professional's role as support staff and their general knowledge of NSSI behaviors is significantly diminished due to mediation of professional's familiarity with various mental health disorders. Sobel calculations were conducted to determine if the indirect effect was significant. The Sobel statistic indicates the indirect effect is significant $(Z=-2.02, p<.043)$. This suggest that familiarity with various mental health disorders significantly mediates the relationship and accounts for variance in professional's general knowledge of NSSI behaviors while controlling for their role as support staff. 
Figure 22

Path Analysis Model for School and District Administrators' General Knowledge of NSSI Behaviors as Mediated by Familiarity with Various Mental Health Disorders

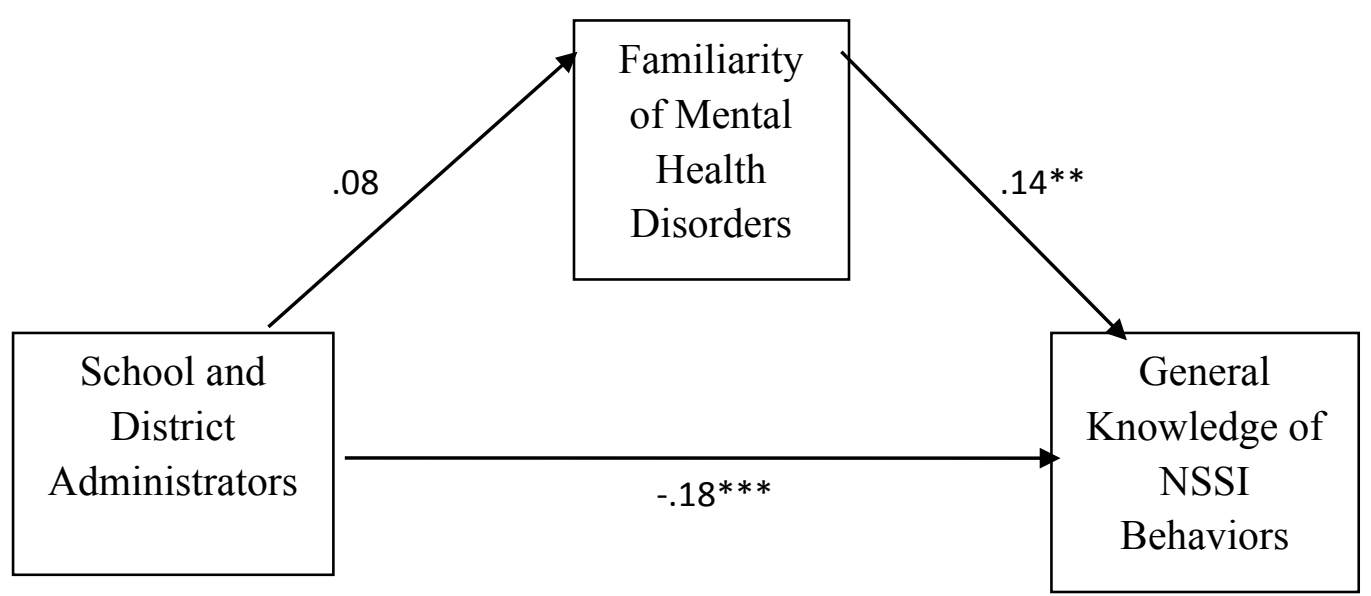

Figure 22 represents a nonsignificant, unmediated model. This suggests the relationship between professional's role as a school or district administrator and their general knowledge of NSSI behaviors is not significantly diminished due to mediation by professional's familiarity with various mental health disorders. The direct effect remains significant in the presence of the mediator. Sobel calculations were conducted to determine if the indirect effect is significant. The Sobel statistic indicates the indirect effect is not significant $(\mathrm{Z}=1.28, \mathrm{p}<.199)$. 
Figure 23

Path Analysis Model for School Nurses' General Knowledge of NSSI Behaviors as Mediated by Familiarity with Various Mental Health Disorders

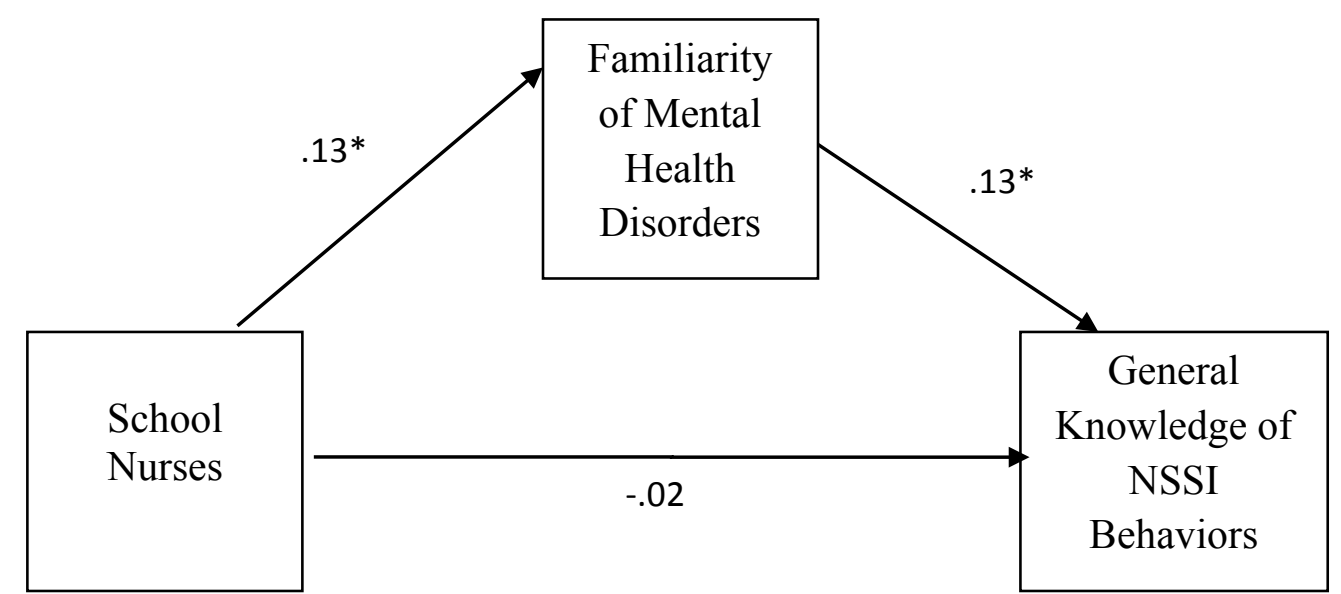

Figure 23 represents a nonsignificant, mediated model. This suggests the

relationship between professional's role as a school nurse and their general knowledge of NSSI behaviors is significantly diminished due to mediation by professional's familiarity with various mental health disorders. Sobel calculations were conducted to determine if the indirect effect is significant. The Sobel statistic indicates the indirect effect is not significant $(Z=1.71, \mathrm{p}<.087)$. This suggests that although the mediated relationship significantly diminishes the direct effect, the indirect effect or mediated relationship does not offer any plausible explanatory power to the model. 
Figure 24

Path Analysis Model for Special Education Teachers' General Knowledge of NSSI Behaviors as Mediated by Familiarity with Various Mental Health Disorders

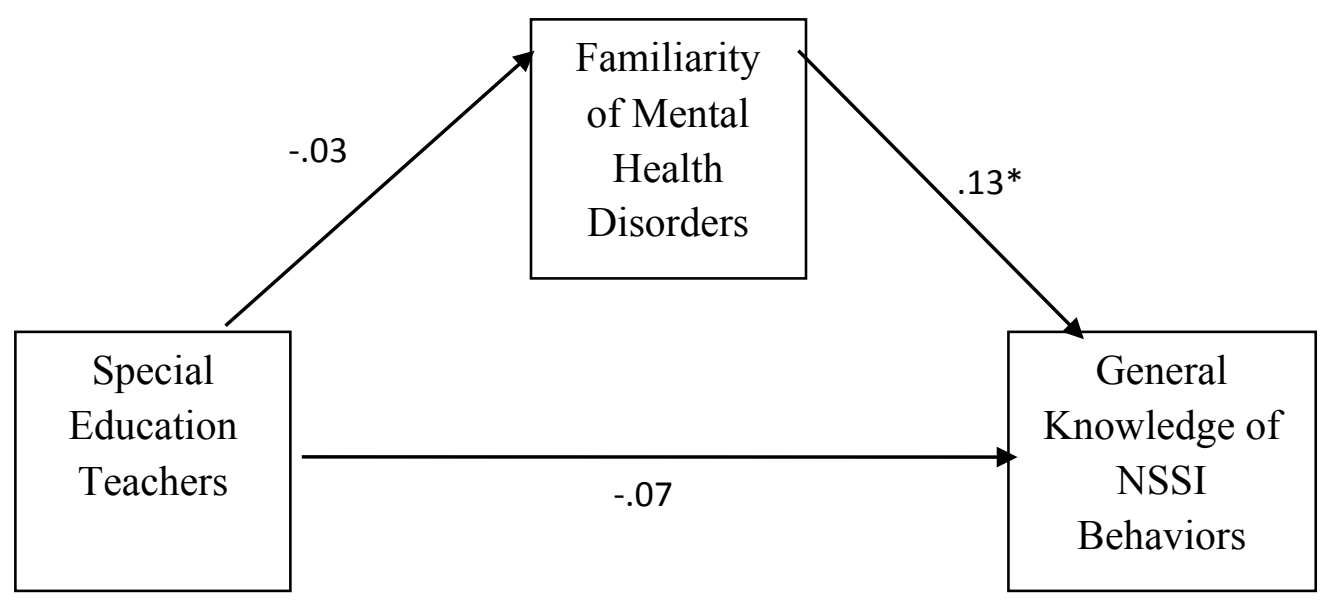

Figure 24 represents a nonsignificant, unmediated model. This suggests the relationship between professional's role as a mental health and counseling associate and their general knowledge of NSSI behaviors is not significantly diminished due to mediation by professional's familiarity with various mental health disorders. Sobel calculations were conducted to determine if the indirect effect is significant. The Sobel statistic indicates the indirect effect is not significant $(Z=-.50, p<.617)$. 
Table 26

Standardized Direct and Indirect Effects for Role of School-Based Professional on General Knowledge of NSSI Behaviors and Perceived Self-Efficacy

\begin{tabular}{|c|c|c|c|c|c|c|}
\hline \multirow{3}{*}{10.5} & \multicolumn{6}{|c|}{ Dependent Variables } \\
\hline & \multirow{2}{*}{$\begin{array}{c}\text { Direct } \\
\text { Experience } \\
\text { with NSSI } \\
\text { Behaviors } \\
\text { (Mediator \#1) } \\
(\mathrm{M} \# 1) \\
\end{array}$} & \multirow{2}{*}{$\begin{array}{l}\text { Familiarity } \\
\text { with Mental } \\
\text { Health } \\
\text { Disorders } \\
\text { (Mediator \#2) } \\
\text { (M\#2) }\end{array}$} & \multicolumn{2}{|c|}{$\begin{array}{c}\text { General } \\
\text { Knowledge of } \\
\text { NSSI Behaviors } \\
\end{array}$} & \multicolumn{2}{|c|}{$\begin{array}{l}\text { Perceived Self- } \\
\text { Efficacy }\end{array}$} \\
\hline & & & $\begin{array}{l}\text { Via } \\
\text { M\#1 }\end{array}$ & $\begin{array}{l}\text { Via } \\
\mathrm{M} \# 2\end{array}$ & $\begin{array}{l}\text { Via } \\
\text { M\#1 }\end{array}$ & $\begin{array}{l}\text { Via } \\
\mathrm{M} \# 2\end{array}$ \\
\hline \multicolumn{7}{|l|}{ Special Education } \\
\hline \multicolumn{7}{|l|}{ Teacher } \\
\hline Direct & $.13 *$ & .03 & $.07^{-.06}$ & - & \multicolumn{2}{|c|}{$.10^{*} \quad-.15^{* *}$} \\
\hline Indirect & - & - & .02 & .00 & $.07^{*}$ & $\begin{array}{r}-.15^{* *} \\
01\end{array}$ \\
\hline Mediator \#1 & & & \multicolumn{2}{|c|}{$.12 *$} & \multicolumn{2}{|c|}{$.54 * * *$} \\
\hline Mediator \#2 & & & \multicolumn{2}{|c|}{$.13^{*}$} & \multicolumn{2}{|c|}{$.41 * * *$} \\
\hline \multicolumn{7}{|l|}{ School Social } \\
\hline \multicolumn{7}{|l|}{ Worker } \\
\hline Direct & $.44 * *$ & $.24^{* * *}$ & \multicolumn{2}{|c|}{$\begin{array}{c}.14^{*} \\
.15^{* *}\end{array}$} & $.28^{* *}$ & $.39 * * *$ \\
\hline Indirect & - & - & .03 & .02 & $.19^{* * *}$ & $.08 * * *$ \\
\hline Mediator \#1 & & & \multicolumn{2}{|c|}{.07} & & $3 * * *$ \\
\hline Mediator \#2 & & & & & & $2 * * *$ \\
\hline School & & & & & & \\
\hline Nurse & & & & & & \\
\hline Direct & .04 & $.13^{*}$ & -.01 & -.02 & -.04 & -.08 \\
\hline Indirect & - & - & .01 & .02 & .02 & $.05^{*}$ \\
\hline Mediator \#1 & & & & & & $* * *$ \\
\hline Mediator \#2 & & & & & & $2 * * *$ \\
\hline School/District & & & & & & \\
\hline Administrator & & & & & & \\
\hline Direct & .08 & .08 & & * - & .02 & -.01 \\
\hline Indirect & - & - & .01 & .01 & .04 & .03 \\
\hline Mediator \#1 & & & & & & $5 * * *$ \\
\hline Mediator \#2 & & & & & & $2 * * *$ \\
\hline Support & & & & & & \\
\hline Staff & & & & & & \\
\hline Direct & $-.17 * *$ & $-.23^{* * *}$ & -.01 & -.00 & $-.12 *$ & $-.12 *$ \\
\hline Indirect & - & - & -.02 & $-.03 *$ & $-.09^{* *}$ & $-.09 * * *$ \\
\hline Mediator \#1 & & & & & & $3 * * *$ \\
\hline Mediator \#2 & & & & & & $* * *$ \\
\hline Mental Health/ & & & & & & \\
\hline Counseling & & & & & & \\
\hline Professional & & & & & & \\
\hline Direct & $.13^{*}$ & $.15^{* *}$ & .01 & .01 & $-.11 *$ & $.12 *$ \\
\hline Indirect & - & - & .01 & .02 & $.07 *$ & $.06^{* *}$ \\
\hline Mediator \#1 & & & & & & $4 * * *$ \\
\hline Mediator \#2 & & & & & & **** \\
\hline
\end{tabular}


Direct Effects of Predictors on Criterion Variables. Table 26 presents direct effects of role of school-based professional on dependent variables. An examination of direct effects revealed general knowledge of NSSI behaviors was predicted by school social workers $(\beta=.17, \mathrm{p}<.002)$ and school/district administrators $(\beta=-.17, \mathrm{p}<.002)$. When examining mediating effects on general knowledge of NSSI behaviors, direct experience with NSSI significantly predicted unit score changes for special education teachers $(\beta=.12, \mathrm{p}<.025)$, support staff $(\beta=.13, \mathrm{p}<.021)$, school nurses $(\beta=.13, \mathrm{p}<$ $.017)$, school/district administrators $(\beta=.15, \mathrm{P}<.008)$, and mental health and counseling associates $(\beta=.13, \mathrm{p}<.020)$. The mediator familiarity with mental health disorders significantly predicted general knowledge of NSSI behavior scores for special education teachers $(\beta=.12, \mathrm{p}<.025)$, support staff $(\beta=.13, \mathrm{p}<.021)$, school nurses $(\beta=.13, \mathrm{p}<.017)$, school/district administrators $(\beta=.15, \mathrm{P}<.008)$, and mental health and counseling associates $(\beta=.13, \mathrm{P}<.020)$. These findings suggest that school/district administrators, when compared to regular education teachers, achieved significantly lower general knowledge of NSSI behaviors when unmediated.

Perceived self-efficacy was predicted by school social workers $(\beta=.47, \mathrm{p}<.000)$, special education teachers $(\beta=-.17, \mathrm{P}<.002)$, support staff $(\beta=-.21, \mathrm{p}<.000)$, and mental health and counseling associates $(\beta=.18, \mathrm{p}<.001)$. Direct experience with NSSI behaviors significantly predicted levels of perceived self-efficacy for all school-based professionals at the $\mathrm{p}<.000$ level. The mediator familiarity with various mental health disorders also significantly predicted levels of perceived self-efficacy for all school based professionals at the $\mathrm{p}<.000$ level, with the exception of support staff $(\mathrm{p}<.023)$. These findings suggest that special education teachers and support staff achieved significantly 
lower perceived self-efficacy scores than regular education teachers. Conversely, all school-based professionals achieved significantly higher perceived self-efficacy scores than regular education teachers when mediated by direct experience with NSSI behaviors and familiarity with various mental health disorders.

Familiarity of various mental health disorders was predicted by a number of school-based professionals. School nurses $(\beta=.13, \mathrm{p}<.016)$, school social workers $(\beta=$ $.24, \mathrm{p}<.000)$, support staff $(\beta=-.23, \mathrm{P}<.000)$, and mental health and counseling associates $(\beta=.15, \mathrm{p}<.006)$ predicted professional's familiarity of various mental health disorders. This finding suggest that support staff's familiarity with various mental health disorders resulted in significantly lower numbers when compared to regular education teachers. While school nurses, school social workers, and mental health counseling associates reported significantly higher numbers of mental health disorders for which they are familiar when compared to regular education teachers.

School-based professionals' direct experience with NSSI behaviors was predicted by school social workers $(\beta=.44, \mathrm{p}<.000)$, special education teachers $(\beta=-.13, \mathrm{P}<$ $.018)$, support staff $(\beta=-.17, \mathrm{p}<.002)$, and mental health and counseling associates $(\beta=$ $.13, \mathrm{p}<.019)$. These findings suggest that special education teachers and support staff reported less direct experience with NSSI behaviors when compared to regular education teachers. On the other hand, school social workers and mental health and counseling associates reported significantly greater direct experience working with individuals that engage in NSSI behaviors when compared to regular education teachers.

Indirect Effects of Predictors on Criterion Variables. Table 26 presents indirect effects of role of school-based professional on criterion variables. Indirect effects 
represent the product of direct path coefficients for the predictor variable to the mediator then the mediator to the criterion variable. Direct experience with NSSI behaviors and familiarity with various mental health disorders serve as mediating variables between school-based professional's role and their general knowledge of NSSI behaviors and perceived self-efficacy.

A number of school-based professionals significantly predicted their direct experience with NSSI behaviors which subsequently predicted their perceived selfefficacy scores all while controlling for their role in the school. School social workers ( $\beta$ $=.19, \mathrm{p}<.001)$ predicted higher levels of direct experience with NSSI behaviors, which predicted higher perceived self-efficacy scores. This pattern was also observed for special education teachers $(\beta=.07, \mathrm{p}<.05)$ and mental health and counseling associates $(\beta=$ $.07, \mathrm{p}<.05)$. These significant findings suggest when compared to general education teachers, school social workers, special education teachers, and mental health and counseling associates average higher perceived self-efficacy scores in working with students that engage in NSSI behaviors when mediated by their level of direct experience with NSSI behaviors. Conversely, support staff obtained significantly lower perceived self-efficacy scores when mediated by their direct experience with students that engage in NSSI behaviors $(\beta=-.09, \mathrm{p}<.01)$ compared to general education teachers.

When accounting for mediating effects on professionals' perceived self-efficacy, familiarity with various mental health disorders significantly predicted scores for school social workers $(\beta=.08, \mathrm{p}<.001)$, school nurses $(\beta=.05, \mathrm{p}<.05)$, and mental health and counseling associates $(\beta=.06, \mathrm{p}<.01)$. These professionals obtained higher scores, compared to general education teachers, of perceived self-efficacy when their familiarity 
with various mental health disorders mediated their role. However, the opposite was observed for support staff $(\beta=-.09, \mathrm{p}<.001)$. Support staff generally reported lower levels of perceived self-efficacy and more so when they are less familiar with mental health disorders when compared to general education teachers.

Lastly, surprising findings were observed with the general knowledge of NSSI behaviors outcome variable and both mediators. Although, the mediators may have contributed to more explanatory power for professional's perceived self-efficacy, they did little to nothing for professional's general knowledge of NSSI behaviors. In fact, only one indirect effect was significant across all professionals. Support staff $(\beta=-.03, p<$ .05) obtained significantly lower general knowledge of NSSI behavior scores and to a lesser degree when mediated by familiarity with various mental health disorders when compared to general education teachers. There were no significant indirect effects mediated by direct experience with NSSI behaviors. This suggests that this mediator offers no further unique prediction power in the path analysis for all school-based professionals. Other implications of this finding are discussed in the following chapter.

\section{Informal Qualitative Analysis}

By far the most frequently ( $\mathrm{N}=27,32 \%)$ shared comment among school-based professionals was the need and/or want of more training and information regarding NSSI behaviors. For example, one professional stated that, "[h]elping school staff understand this concept and how to intervene is VERY needed!” This was the consensus for identified mental health and non-mental health (e.g., teachers) professionals. One nonmental health professional stated, “[a]s a classroom teacher, I don't feel I have the 
training to work with students who are engaging in self-injurious behaviors." Three of the 27 professionals stated they need more training in mental health issues in general. A professional indicated they "would love to have staff development on dealing with students mental health."

The next comment frequently made by professionals was to refer the student engaging in NSSI behaviors to a school-based mental health professional (i.e., school counselor, school psychologist, school social worker) $(\mathrm{N}=15 ; 18 \%)$. Some professionals indicated that school counselors and school social workers could intervene with students that engage in NSSI behaviors more appropriately. This was evident with some professionals who considered NSSI behaviors a specialty area of theirs $(\mathrm{N}=6 ; 7 \%)$ and some social workers provided professional development $(\mathrm{N}=2 ; 2 \%)$.

An equally frequent comment was the number of professionals who indicated they knew multiple students, at time in excess of 20, who have engaged in the behavior or who are currently engaging in the behavior $(\mathrm{N}=15 ; 18 \%)$. One professional confessed they "have only encountered one confessed incident. I am wondering how many I have missed." Here the professional recognizes that this may be a more common occurrence than expected, but also recognized there is a need to know how to recognize individuals that engage in NSSI behaviors in the student population. However, some professionals shared they currently work with students that engage in NSSI behaviors $(\mathrm{N}=5 ; 6 \%)$. Though this is a small number of individuals who identify themselves as professionals who currently work with students that engage in NSSI behaviors, there are a number of professionals who stated they would work with students, if they knew what interventions to provide $(\mathrm{N}=11 ; 13 \%)$. 
There appeared to be some dissatisfaction with community-based resources $(\mathrm{N}=4$; 5\%). Some professionals indicated they were ineffective and did not adequately address NSSI behaviors. However, professionals indicated there is not enough school-based staff to address NSSI behaviors in the school $(\mathrm{N}=3 ; 4 \%)$. Furthermore, if professionals were to intervene, they feel stifled about what to recommend or services to provide due to constraints of financial responsibility for various services by the district $(\mathrm{N}=4 ; 5 \%)$. The availability of quality school- and community-based resources conundrum may be an issue in and of itself worthy of more attention.

Another recommendation was to educate and involve families ( $\mathrm{N}=6,7 \%)$. Professionals indicated the importance of family involvement in their comments. For example, one professional stated that they would "refer to school outreach counselor and/or notify parents and advise counseling." Another individual stated that they are "able to listen, contact parents, be available as a resource..." However, some professionals view families as barriers and potential catalyst for NSSI behaviors $(\mathrm{N}=4$; 5\%). One professional stated, "Many parents believe a child can just "Say No" to cutting etc. I deal with many emotionally immature parents who have limited emotional resources to deal with the issue. I usually make a child protective referral as that agency routinely provides additional motivation for parents to follow through with treatment." Another professional stated they "found these incidents more common when the children are unsupervised in the home, and when alcohol is involved." Clearly, there are some misconceptions regarding self-injury even though there may be a kernel of truth to the 
comments. Nevertheless, for some professionals, families are a source of stress and can exacerbate NSSI behaviors in their students.

Some professionals shared they have personal experiences with NSSI behaviors $(\mathrm{N}=3 ; 4 \%)$ whether by engaging in NSSI behaviors themselves or dealing with their own children engaging in the behavior. This was to be expected given the number of individuals that engage in this behavior in the general population. Other professionals indicated they do not know what to do to help students that engage in NSSI behaviors $(\mathrm{N}=2 ; 2 \%)$. Others feel that NSSI behaviors and interventions are beyond the scope of their current knowledge and/or role $(\mathrm{N}=12 ; 14 \%)$. While others indicated they do not see NSSI behaviors at all $(\mathrm{N}=4 ; 5 \%)$ due to their role (e.g., support staff) or grade placement (e.g., early childhood). One support staff professional indicated that students that engage in NSSI behaviors should have a trusted adult to talk to $(\mathrm{N}=3 ; 4 \%)$.

Other, less frequently mentioned recommendations and/or comments shared were involve the school principal when working with students that engage in NSSI behaviors $(\mathrm{N}=1 ; 1 \%)$; primarily seen in secondary grades $(\mathrm{N}=4 ; 5 \%)$; monitor health issues and wounds $(\mathrm{N}=2 ; 2 \%)$; serve on a multidisciplinary team $(\mathrm{N}=1 ; 1 \%)$; special education is not a satisfactory option for students engaging in NSSI behaviors ( $\mathrm{N}=1 ; 1 \%)$; online resources were found to be helpful (e.g., Cornell University website) $(\mathrm{N}=1 ; 1 \%)$; and school social worker's limited use of credentialing to provide need services to students who engage in NSSI behaviors $(\mathrm{N}=5 ; 3 \%)$.

The qualitative portion of the instrument also asked for comments regarding the survey instrument. Five professionals (6\%) indicated that the survey response options were limited and did not allow them to full articulate their opinions, attitudes, and 
knowledge regarding NSSI behaviors. These comments are taken into advisement for future studies and will be used to refine the current instrument. 


\section{CHAPTER SIX}

\section{DISCUSSION}

Non-suicidal self-injury is on the rise among children and adolescents (D' Onofrio, 2007). It involves a number of bodily harm acts to the individual and often committed covertly. Results of NSSI behaviors often leave permanent scars both physically and emotionally. School-based professionals are on the frontlines to intervene with students that engage in NSSI behaviors and can attempt to prevent its occurrence (or recurrence). It is important that school-based professionals are properly trained to recognize the signs of individuals engaging in NSSI behaviors. Equally important is the perceived self-efficacy of school-based professionals when providing recommendations and interventions to students engaging in NSSI behaviors.

Findings from this study indicate significant differences in general knowledge of NSSI behaviors and various school-based professionals. An examination of mean differences indicates that school social workers have more general knowledge $(\mathrm{p}<.007)$ of NSSI behaviors than school/district administrators. Furthermore, familiarity with various mental health disorders was a significant mediator for school social workers when predicting their general knowledge of NSSI behaviors score when compared to general education teachers. This supports the first study hypothesis. School social workers obtaining higher general knowledge of NSSI behavior scores is not surprising as they typically receive more training in mental health issues than other non-mental health school-based professionals.

While mental health and counseling associates are typically possess specific training in and knowledge of mental health issues, they did not achieve general 
knowledge scores that were exceptionally different from other school-based staff. In fact, there were no other statistically significant differences in general knowledge of NSSI behaviors among the other school-based professionals. Consistent with previous research (Best, 2005; Moore, 2009; Walter, Gouze, \& Lim, 2006), most school-based staff achieved mean scores that were approximately half of the maximum knowledge score. This suggests there is no school-based professional that holds an exceptional amount of knowledge with respect to NSSI behaviors.

The second hypothesis predicted that school-based staff that have familiarity with various mental health disorders, woould have significantly greater perceived selfefficacy. An examination of mean differences between school-based staff reveals that school social workers have more $(\mathrm{p}<.007)$ familiarity with various mental health disorders than regular education teachers, school nurses, and support staff. Additionally, mental health and counseling associates have more familiarity with mental health disorders than all other professionals.

Overall, school social workers obtained the highest perceived self-efficacy scores. They were statistically and significantly different from all school-based professionals with the exception of mental health and counseling associates and school/district administrators. When examining mediating effects, both mediators significantly $(\mathrm{p}<.001)$ mediated the relationship between school social workers and their perceived selfefficacy. This suggest that school social workers direct experiences with NSSI behaviors and their familiarity with various mental health disorders contributes to their confidence in working with students who may engage in NSSI behaviors despite their limited general knowledge. The second hypothesis is fully supported. 
Lastly, the third hypothesis examines direct experience with NSSI behaviors and familiarity with mental health disorders as mediating variables. Does direct experience with NSSI behaviors mediate the relationship between school-based professionals' role and their general knowledge of NSSI behaviors? Surprisingly, it does not mediate this relationship for any school based-professional. This finding may suggest that professional's direct experiences with students that engage in NSSI behaviors may not have reached the desired outcome. Further, more information and resources may have been needed for professionals to effectively intervene as noted in the qualitative section of this study. Consequently, their experience did not enhance their current knowledge of NSSI behaviors. This does not support hypothesis three.

Does direct experience mediate the relationship between school-based professionals' role and their perceived self-efficacy? Yes. School social workers, special education teachers, support staff, and mental health and counseling associates achieved higher, or lower for support staff, perceived self-efficacy scores when factoring in their direct experience with NSSI behaviors. This finding does partially support hypothesis three. This suggest that some professionals who have previous experience with students that engage in NSSI behaviors may feel more efficacious working with other students engaging in NSSI behaviors in the future. However, it is difficult to conclude that this finding is due to more knowledge of NSSI behaviors or their comfort level in broaching the topic and offering support and resources. More than likely, it is the latter as evidenced in the qualitative section of this study.

Lastly, it was hypothesized that familiarity with various mental health disorders will mediate general knowledge of NSSI behaviors and predicted perceived self-efficacy 
scores. This hypothesis was fully supported. Similar to other findings, school social workers reported higher numbers of mental health disorders with which they are familiar. However, after examining indirect effects, familiarity with various mental health disorders did not predict general knowledge of NSSI behaviors for most school-based professionals with the exception of support staff. This suggests support staff have considerably less knowledge of various mental health disorders than most school-based professionals and general knowledge of NSSI behaviors scores that are less than regular education teachers.

Considerably more significant findings were observed in mediated relationships between school-based professional's role and their perceived self-efficacy especially for mental health professionals. Mental health professionals such as school social workers and mental health/counseling associates may be aware of various mental health disorders in general and NSSI more specifically through their role in the school as evidenced in their higher general knowledge scores. This rings true more so for school social workers. However, mental health training for professional's current role in the school is different for non-mental health professionals. Nonetheless mental health training is equally important as seen in the qualitative comments. Mental health training may not be readily available to other school-based professionals such as regular education teachers, special education teachers, school/district administrators, and support staff. For example, professionals indicated they would involve the principal when dealing with students that engage in NSSI behaviors. Some professionals stated that intervening with students is currently beyond their current role. Though this may be true, most school-based professionals recognize that training specific to NSSI behaviors is beyond their current 
role, but acting in crises goes across school-based roles and they may be the one called to intervene.

In addition, it is important to explore in-service training opportunities, school policies, and curricula of all school-based professionals within various schools and across districts to gather a larger picture of this phenomenon. This is especially true for support staff. In this study, the support staff comprised of paraprofessionals, media specialists, speech pathologists, and other specialists reported feeling less efficacious intervening with students engaging in NSSI behaviors. However, these vital staff may be a trusted adult for some students. Additionally, they are on the frontlines working with students due to their relationship that may be on a one-on-one basis.

School nurses had relatively high, though not statistically significant, general knowledge scores compared to other non-mental health professionals. This finding supports other literature findings that school nurses are trained in responding to crises and play a very important role in carrying out safety plans (Shapiro, 2006). Along these lines, qualitative comments in the study revealed that nurses look to treat overall health issues and, if present, wounds to prevent infections for students engaging in NSSI behaviors. However, school nurses felt less efficacious working with students that engage in NSSI behaviors compared to school social workers and mental health and counseling associates. This may suggest their comfort level lies in the treatment of physical symptoms rather than emotional components of NSSI.

The current study delved into a suggested area of exploration from the previous study, perceived self-efficacy, and found a gold mine. Findings in this study and others have shown that school-based professionals and particularly mental health professionals 
may feel "ill at ease" when intervening with students that engage in self-injurious behavior (Best, 2005). According to qualitative information provided in the study, many professionals indicated they would refer the student elsewhere for needed services. This further supports the need for professional development in this area as well as the creation of a multidisciplinary crisis teams to address the growing incidence of students engaging in NSSI behaviors.

\section{Implications}

The current instrument demonstrated moderate reliability for the general knowledge of NSSI behaviors subsection $(\alpha=.612)$. Although a slightly less desirable reliability coefficient was achieved, for a perception and feeling measure, it is approaching an acceptable range $(\alpha=.70)($ Kaplan $\&$ Saccuzzo, 2001). Therefore, conclusions can be drawn exercising a degree of caution when generalizing study findings about school-based professionals' general knowledge of NSSI behaviors. The low reliability finding could also be contributed to various other constructs, other than general knowledge, that were measured. The perceived self-efficacy section of the instrument achieved an acceptable level of reliability and therefore can be interpreted with a significant degree of confidence.

While the data indicate some significant differences, in various directions, in general knowledge of NSSI behaviors, it should be noted no school-based professional group obtained scores within very knowledgeable levels (scores 36 or higher). This suggests that most individuals in the study do not possess a great amount of knowledge regarding NSSI behaviors. This is surprising given the number of students engaging in 
this behavior. The prevalence rates of self-injury illustrate the importance of increasing the knowledge base of school-based professionals. Similar results have been found across various studies examining the perceptions of teachers, health professionals, hospital staff, and clinical professionals such as psychologists (Best, 2005; Heath, Toste, \& Beetam, 2006; Huband \& Tatum, 2000). Because none of the school professionals are exceptionally knowledgeable with NSSI behaviors, more subtle and/or complex aspects of NSSI are even more likely to be misunderstood, such as how to differentiate selfinjury from suicidal gestures, copycat cutting or other environmental factors. The latter was evident in the qualitative comments regarding parents who indulged in alcohol and did not monitor their children. While delinquency may be a risk-factor for the student growing up in the home, NSSI behaviors is not necessarily an expected practice.

School psychologists have a general understanding of psychiatric underpinnings of mental health disorders, which may include NSSI behaviors (Best, 2005). Similar training backgrounds are expected for school social workers and some school counselors. Pilot and current study results suggest that school-based mental health professionals are in a unique position to serve as leaders of interagency and multidisciplinary teams addressing mental health in their schools (Best, 2005). Alas, this finding may be a truth for some, rather than the norm for all school-based mental health professionals.

Accessible and practical evidence-based interventions that can be implemented efficiently were cited as a need. This is also supported by a study that found clinical staff (e.g., psychiatric nurses, psychologists, and non-psychiatric nurses) are in need of more evidence-based interventions (Huband \& Tatum, 2000). Furthermore, findings suggest there should be opportunities for in-service trainings or professional development 
sessions for school-based professionals around NSSI behaviors. As with the pilot study, school-based mental health professionals, to whom other school-based professionals stated they would refer students who engage in NSSI behaviors, have slightly more knowledge than other professionals. Also, school-based mental health professionals' perceived self-efficacy is not proportional to their knowledge of NSSI behaviors. Therefore, the "experts" in the school are also crying out for information and training as seen the current study's qualitative comments and findings. Both knowledge of NSSI behaviors and perceived self-efficacy presents school-and district wide issues that should be addressed appropriately.

Equally critical is access to resources not only outside of the school, but also within the school, particularly if the behaviors are occurring in school (Best, 2005). Intraand interagency teams contribute to the successful delivery of effective and efficient services. Agencies may include local hospitals, self-injurious behaviors advocacy groups (e,g., S.A.F.E), psychologists, and psychiatrists (Best, 2005). Access to quality services poses a significant problem to schools that are strapped for resources. However, given the low number of mental health professionals that participated in the study, this may be more than an indication of participation rather a lack of presence in the schools solicited.

It is extremely concerning when there is a lack of credentialed and appropriately trained staff to provided services to students who engage in NSSI behaviors. What should a school and/or district do in this case? Available staff should be trained and partnerships with community-based agencies known for providing quality services should be created. Because non-suicidal self-injurious behaviors may occur at school or at home, it is a community issue and should be addressed by all key stakeholders. Schools may not have 
to be the sole provider of services, but they should have an action plan in place that describes in detail what to do in crisis.

\section{Limitations}

The results of the present study are intriguing but have their share of limitations that should be considered for future research in this area. These limitations should spark conversations among school-based professionals regarding the challenges of addressing NSSI behaviors and mental health issues in general. There are four major areas of limitations in this study: sample population and sampling methodology, unidentified mediators, ordering of mediators, and the developed instrument.

First, there were a number of sample-related issues that limit the interpretation of results and the degree to which results can be generalized to larger populations of each group. One of which is unequal cell sizes used in the data analysis for the current study. The sample population of school-based professionals was skewed more toward school educators when compared to observed sample size (n) across other school-based professionals. Recruitment techniques (e.g., soliciting participants through professional and district listservs or mass emailings) proved inadequate to increase the number of mental health professionals and school administrators participating in the study. However, collapsing across school-based mental health professionals proved advantageous in increasing the $\mathrm{n}$ for this group for comparisons purposes.

The number of cases in the seven groups was very different, with an overwhelming number of respondents representing teachers (both general and special education). More data should be collected for all school-based mental health professionals, school nurses, and school administrators to make observed $\mathrm{n}$ equitable to 
the number of cases of educators. This is crucial to ensure generalizability of study conclusions to broader populations of school-based professionals.

Another limitation of the current sample is the survey participant selection. The researcher used a convenience sample in this study that did not allow for random selection of participants or exploration of other venues for participant recruitment. This may slant the results to those who are motivated to participate in a study regarding nonsuicidal self-injurious behaviors and who may want more information regarding NSSI and students that engage in NSSI behaviors.

Next, there are other variables that may serve as mediators in the study that were not measured. Various survey questions may have provided further explanation for differences in knowledge and self-efficacy among the various school-based professionals. For example, "How frequently do you see students engaging in NSSI behaviors in your school?" and "How long, in years, have you been in your current role?" may have mediated the relationship between role of the professionals and perceived self-efficacy. This would allow the study to determine other significant factors in the proposed causal relationship model and potentially reduce the amount of unexplained error associated with the outcome variables.

Similarly, school-based professionals with frequent contact with students that engage in NSSI may have moderated the effect on general knowledge and perceived selfefficacy. Potentially, this variable may have strengthened the relationship between school-based professional's role and general knowledge and perceived self-efficacy. Explaining the significance of this finding is the core of this study--identifying factors that contribute to an increase of knowledge and self-efficacy to better assist school-based 
professionals to help students. Further, this additional statistical analysis provides supplementary information that could be used to recruit specific school-based professional for models as suggested by SCT.

Another interesting question would have been, "Have you received any training, professional development, or information regarding NSSI behaviors?" Though similar to the experience with NSSI question, it provides further distinction by examining prior formal training of school-based professionals with respect to NSSI behaviors. Previous training in NSSI may have also functioned as a moderator in the current study. Moreover, this variable would have fit well in the overall meditational model. For example, unexplained variance associated with outcome variables general knowledge and perceived self-efficacy may have been reduced with this nuance variable.

Ordering of current study variables was a hindsight mishap. According to the current literature, the study's model proposed a seemingly logical ordering of mediators, direct experience with NSSI behaviors and familiarity with various mental health disorders, to predict perceived self-efficacy and general knowledge of NSSI behaviors. However, it is equally plausible to assume that general knowledge of NSSI behaviors predicts perceived self-efficacy and direct experience with NSSI behaviors. Future research studies would be greatly strengthen by proposing and testing several models to explain perceived-self-efficacy and the knowledge base of school-based with respect to NSSI behaviors.

The developed measure lacked questions focusing on the impact of NSSI behaviors on individual and classroom learning. In the knowledge of interventions section of the survey, professionals were asked to indicate the various methods they 
would employ to assist students who engage in NSSI behaviors. The percentage of professionals that indicated they "would not refer students for special education services" was high though not surprising. This endorsement was more than likely due to their knowledge of IDEIA regulations stating an adverse impact on the student's ability to learn must be evident for services to be considered (IDEIA, 2004). The question in the survey was not clear in detailing if the student met this crucial criterion. Therefore, it is presumptuous to conclude that school-based professionals are not generally knowledgeable of school-based interventions for students that engage in NSSI behaviors. In sum, the aforementioned statement on the survey lacked a qualifier and important distinction that may have led to different results regarding the general knowledge of school-based professionals. On the other hand, perhaps it is both their perceived and actual role in the school that may have influenced their responses to questions in this section of the survey.

The measure contained language that may have been confusing and convoluted, making participants more likely to respond "Neutral" or "Do Not Know." This may also be a direct result of the terminology used in common versus clinical circles. For example, in more common circles, individuals may use "cutting." While in research and clinical settings, the terms self-injury, self-mutilation, self-harm, or non-suicidal self-injury may be used instead. The seriousness and the intent of the act may not be conveyed using inconsistent terminology. It is more politically correct to use NSSI. However, within this particular construct, further distinction between what is and is not considered to be suicidality and is somewhat convoluted. 
Additionally, reliability was lower than desired for the current measure. For optimal results, the reliability of an attitude measure should be in the .70 and above range for instruments that measure attitudes and beliefs (Kaplan \& Saccuzzo, 2001). Wording of instrument items may have contributed to low reliability. Other contributing factors were the presentation of the survey online, participant fatigue given the length of the instrument, answer choices, and subject matter. One way to increase reliability of the instrument is to alter the response choices to reflect knowledge rather than attitudes. Response choices should have been on a "True/Yes" of "False/No" or "I Don't Know" rather than the present attitude scale. Although addressed for data analysis, the true goal of the instrument was to assess knowledge and other factors may have been measured. Ultimately, this may have impacted the degree to which results adequately addressed the study's research questions.

Finally, since the primary use of study findings was to inform training practices of school-based professionals to effectively work with students that engage in NSSI behaviors, a needs assessment could have been incorporated into the survey. For training purposes, it is important to ascertain gaps of knowledge and skills to assure information is directed to those areas of need rather than providing a blanket training session. The professional's development and training sessions may look very different across roles, grade levels/schools, and districts. That is the beauty of needs assessments--it determines specific needs of the intended population. Although the survey asked for certain demographic information to distinguish needs across grade levels/schools, roles, and districts, it was voluntary. As a result, self-disclosed knowledge and perceived selfefficacy may be over- or underestimated. This may be in part due to the nature of the data 
collection method (anonymous online survey) and sharing of data (districts were offered a professional development opportunity in exchange for their participation relative to their district findings).

\section{Future Directions}

Given the limitations above, it is research worthy to improve on the measure developed for this study. Due to the low reliability of the measure, it is suggested that items be subjected to a rigorous item analysis and other measures be consulted to aid in the development of items. The current measure contains information that is useful for professional development planning. Additionally, the current measure could be used as a needs assessment for further focused training for school-based health and mental health professionals. A confirmatory factor analysis may prove useful as well to determine the existence of individual subsections within the measure. It is important to create distinctly

different subsections within the current measure to ensure that each section is measuring what it purports to measure.

Developing a protocol for interdisciplinary teams that include interagency assistance would be useful as well. Although beyond the scope of this study, the nonsignificant results and qualitative information reveal a need within the school for information and involvement. It is important to factor in school-family partnerships for any intervention seeking to address mental health issues. Helping families recognize warning signs of a potential mental health issue and providing community links for ongoing support for their child(ren), would aid in developing potent interventions. 
Based on current study findings, it appears reasonable to suggest that support staff, administrators, and teachers develop collaborative partnerships with health and mental health professionals to address this growing concern. The ideal level of training would increase awareness of mental health in general. This would be followed with multiple training sessions identifying symptoms and characteristics of mental health disorders. Lastly, training sessions would involve brainstorming and planning among all school-based professional as to how the team would address crisis situations that may arise in their school. Out of the sessions, holistic yet focused interventions could be developed as students are identified with behavioral or emotional symptoms of underlying mental health issues. The overarching goals of staff training sessions are increasing awareness, knowledge and preparedness.

With respect to specific training topics, school-based professionals may benefit from professional development in the area of NSSI behaviors and other mental health symptoms. Because this is an exploratory study, additional qualitative data could have been collected regarding the formal and/or informal training opportunities, including coursework, related to NSSI behaviors. According to Shapiro (2008), many school-based health professionals note formal coursework in mental health issues, more specifically, NSSI behaviors. The study also discusses school-based mental health professionals and educators receiving crises intervention training onsite to deal with issues such as selfinjurious behaviors (Shapiro, 2008).

These findings coupled with the current study's results can be used to inform future professional development and training practices at the pre- and in-service level. There are a number of school-based professionals that have little experience with mental 
health disorders in general, much less NSSI behaviors. Results of this study suggest that professionals who have more familiarity with mental health disorders feel more efficacious working with students engaging in NSSI behaviors. Further, some schoolbased professionals have embraced their multi-faceted role and recognize that a great deal of interpersonal skills is required to effectively intervene with students with mental health issues. For example, school-based professionals responding to students engaging in NSSI behaviors should remain calm, normalize students' experiences, and listen effectively to students' concerns (Heath \& Nixon, 2009; Klonsky et al., 2011).

Barriers to training school-based professionals have been noted to include attitudes toward mental health disorders in general, poor collaboration between schoolbased mental health and non-mental health professionals, initial reactions to individuals engaging in NSSI behaviors, and lack of effective listening skills (Heath \& Nixon, 2009; Heath, Toste, Sornberger, \& Wagner, 2011; Reinke et al., 2011). There are numerous methods that may be used to engage school-based staff to break through preconceived notions and misconceptions of NSSI behaviors. The first step is to note that NSSI is a symptom of a much larger mental health issue that is often treated as a fire to extinguish rather than it signifying a more pervasive mental health issue. However, armed with this knowledge, establishing a firm knowledge base of mental health disorders in general may be well received. Current study qualitative results indicate that most school-based professionals want to know more about NSSI and recognize their limited training and knowledge with respect to mental health disorders and particularly NSSI behaviors.

Qualitative findings suggest more information regarding NSSI and its association with common mental health disorders may prove helpful to increase awareness and 
sensitivity to students engaging in NSSI behaviors. Additionally, most school-based professionals indicated that they would utilize a number of interventions that were presented in the survey. It can be concluded school-based professionals would like to draw upon the knowledge and assistance from others to address the needs of students engaging in NSSI behaviors and mental health disorders in general. Moreover, most school-based professionals indicate they would feel more efficacious working in teams to assist students engaging in NSSI behaviors. This is a telling revelation that lends itself quite nicely to Social Cognitive Theory. For example, collective self-efficacy underscores the effectiveness of multidisciplinary teams intervening with students that engage in NSSI behaviors.

The first step is to train school-based staff to respond effectively and efficiently to students who engage in NSSI behaviors. In conjunction, teams are encouraged to recognize some school-based staff are in buildings or roles where they will mostly likely encounter students engaging in NSSI behaviors (e.g., junior high and high school buildings, school nurses). This is due to the NSSI onset characteristics. Ultimately, teams of individuals who possess knowledge and experience can operate within and between buildings to train other school-base professionals to use prevention and early intervention methods in response to mental health crises. Most of these professionals are already part of multidisciplinary teams and have access to a "think tank" of other school-based professionals.

Due to the sensitive nature of the topic, school-based professionals and students alike may shy away from openly discussing the use of NSSI as a coping mechanism. It is important to recognize nuance variables that are specific to school-based professionals 
when they intervene with students that engage in NSSI behaviors. Variables may include attitudes toward mental health issues, responsibility of intervening with students that engage in NSSI, and biases against students that engage in NSSI behaviors. Increasing the knowledge of school-based professionals by addressing common myths and misconceptions of NSSI is critical in fostering compassion and understanding (Heath, Toste, Sornberger, \& Wagner, 2011). Furthermore, this may lead to effective interventions and responses that optimally serve students with mental health issues.

Along those lines, school-based staff may have visceral reactions to wounds, scars, or even recall traumatic events in their past associated with the students engaging in NSSI behaviors (Klonsky et al, 2011). According to SCT, aversive somatic arousal initiated by students engaging in NSSI may impede effective and efficient intervention with students. This may lead to further alienation and distrust of school-based professionals by students. This can be addressed by asking school-based staff to examine their affect and connect those feelings with misconceptions and their perceived selfefficacy. For example, if school-based professionals feel students are engaging in NSSI behaviors strictly for attention, they may be less likely to assist students due to their perceived limited impact on superficial behaviors.

To address these emotions elicited by various behaviors associated with mental health disorders, intrapersonal awareness training may be necessary. This may entail asking staff what they know about NSSI, how they feel about NSSI in general, and are they comfortable intervening with students that engage in NSSI behaviors. As found in the current study, these directly impact the desire to obtain knowledge and skills to work with students that engage in NSSI behaviors. 
School-based professionals' agentic levels should be considered when exploring intrapersonal barriers. It can be concluded that school-based staff's agentic levels are relative to the amount of motivation they have to help students that engage in NSSI behaviors. For instance, if a school-based professional is inclined to address mental health issues in their classroom, they may develop concrete goals to decrease the occurrence of NSSI behaviors for students.

Lastly, personal experiences are powerful and meaningful serving as real-life success stories. They provide opportunities for therapeutic alliance and empathetic listening for others engaging in NSSI behaviors. In the study, some professionals indicated personal experience with NSSI behaviors. Furthermore, these individuals shared how this allows them to connect with students that engage in NSSI behaviors. These personal experiences may increase professional's perceived self-efficacy and task persistence in assisting students who engage in NSSI behaviors. As comfort levels allow, these particular professionals could serve on multidisciplinary teams assisting students in crisis. In sum, school-based professionals may be more important that they initially realize. They often serve as role models and trusted adults to students. Assisting all school-based professionals to step into these roles, for which many have not received any formal training, is uncharted territory. Researchers, educators, and practitioners alike should come on one accord to recognize that socioemotional needs of students are equally important as meeting their academic needs. 


\section{REFERENCES}

Adler, P.A., \& Adler, P. (2007). The demedicalization of self-injury: From psychopathology to sociological deviance. Journal of Contemporary Ethnography, 36, 537-570.

Alderman, T. (1997). The scarred soul: Understanding and ending self-inflicted violence. Oakland: New Harbinger Publications, Inc.

American Academy of Child and Adolescent Psychiatry. (1999, December). Self-injury in adolescents. Retrieved October 08, 2005, from http://www.aacap.org/publications/factsFam/73.htm

American Psychiatric Association. (2000). Diagnostic and statistical manual of mental disorders (4th ed.). Washington D.C.: Author.

Bandura, A. (1982). Self-efficacy mechanism in human agency. American Psychologist, 37, 122-

147.

Bandura, A. (1989). Human agency in Social cognitive theory. American Psychologist, $44,1175-1184$.

Baron, R. M., \& Kenny, D. A. (1986). The moderator-mediator variable distinction in social psychological research: Conceptual, strategic, and statistical considerations. Journal of Personality and Social Psychology, 51, 1173-1182.

Beasley, M.T., \& Sheehan, J.K. (1994). Choosing a MANOVA statistic when covariances are unequal. Paper presented at the Annual Meeting of the Midwestern Educational Research Association in Chicago, IL.

Best, R. (2005). An educational response deliberate self-harm: Training, support, and school-agency links. Journal of Social Work Practice, 19, 275-287. 
Bland, J.M., \& Altman, D.G. (1996). Statistics notes: Transforming data. British Medical Journal, 312, 770 .

Bloom, C. M., \& Holly, S. (2011). Toward new avenues in the treatment of nonsuicidal self-Injury. Journal Of Pharmacy Practice, 24, 472-477.

Bohus, M., Haaf, B., Stiglmayr, C., Pohl, U., Bohme, R., \& Linehan, M. (2000) Evaluation of inpatient dialectical-behavior therapy for borderline personality disorder: A prospective study. Behaviour Research and Therapy, 38, 875-887.

Cassano, P., Lattanzi, L., Pini, S., Osso, L. D., \& Cassano, G. B. (2001). Topiramate for self-mutilation in a patient with borderline personality disorder. Bipolar Disorders, 3, 161.

Center for Disease Control and Prevention. (2007). Suicide trends among youths and young adults aged 10-24 years: United States 1990-2004 (MMWR Vol. 56). Atlanta, GA: Epidemiology Program Office. Retrieved March 17, 2009, from http://www.cdc.gov/mmwr/preview/mmwrhtml/mm5635a2.htm

Childstats.gov (2012). America's children: Key national indicators of well-being, 2013. Retrieved May 19, 2013, from http://childstats.gov/

Claes, L., \& Vandereycken, W. (2007) Self-injurious behavior: Differential diagnosis and functional differentiation. Comprehensive Psychiatry, 48, 137-144.

Claes, L., Houben, A., Vandereycken, W., Bijttebier, P., \& Muehlenkamp, J. (2010). Brief report: The association between non-suicidal self-injury, self-concept and acquaintance with self-injurious peers in a sample of adolescents. Journal Of Adolescence, 33, 775 . 
Cohen, J. (1988). Statistical power analysis for the behavioural sciences $\left(2^{\text {nd }}\right.$ ed.). New York: Academic Press.

Conterio, K. \& Lader, W. (1998). Bodily harm: The breakthrough healing program for self-injurers. New York: Hyperion.

Crawford, T., Geraghty, W., Street, W., \& Simonoff, E. (2003). Staff knowledge and attitudes towards deliberate self-harm in adolescents. Journal of Adolescence, 26, 619-629.

Czarnopys, B. B. (2002). Exploring the razor's edge: Understanding adolescent selfharm. Paradigm, Summer, 12-13 \& 22.

D’Onofrio, A. (2007). Adolescent self-injury: A comprehensive guide for counselors and health care professionals. New York, NY: Springer

Deliberto, T.L., \& Nock, M. K. (2008). An exploratory study of correlates, onset, and offset of non-suicidal self-injury. Archives of Suicide Research, 12, 219-231.

Evans, J., Evans, M., Morgan, H., Hayward, A., \& Gunnell, D. (2005). Crisis card following self-harm: 12-month follow-up of a randomised controlled trial. The British Journal Of Psychiatry: The Journal Of Mental Science, 187, 186-187.

Favazza, A. (1987). Bodies under siege. Baltimore: Johns Hopkins.

Favazza, A., \& Conterio, K. (1989). Female habitual self-mutilators. Acta Psychiatrica Scandinavica, 79, 283-289.

Fernandez-Ballesteros, R., Diez-Nicolas, J., Caprara, G. V., Barbaranelli, C., \& Bandura, A. (2002). Determinants and structural relation of personal efficacy to collective efficacy. Applied Psychology: An International Review, 51, 107-125.

George, D., \& Mallery, P. (2003). SPSS for Windows step by step: A simple guide and 
reference. 11.0 update (4th ed.). Boston: Allyn \& Bacon.

Gilman, R., \& Medway, F. J. (2007). Teachers' Perceptions of School Psychology: A Comparison of Regular and Special Education Teacher Ratings. School Psychology Quarterly, 22, 145-161.

Glassman, L.H., Weierich, M.R., Hooley, J.M., Deliberto T.L., \& Nock, M.K. (2007) Child maltreatment, non-suicidal self-injury, and the mediating role of selfcriticism. Behaviour Research and Therapy, 45, 2483-2490.

Gratz, K.L. (2006). Risk for repeated deliberate self-harm among female college students: The role and interaction of childhood maltreatment, emotional inexpressivity, and affect intensity/reactivity. American Journal of Orthopsychiatry, 72, 128-140.

Hawton, K. (2000). Gender differences in suicidal behavior. British Journal of Psychiatry, 177, 484-485.

Heath, N. L., \& Nixon, M. K. (2009). Assessment of nonsuicidal self-injury in youth. In M.K. Nixon and N.L. Heath (Eds.), Self-injury in youth: The essential guide to assessment and intervention (pp.143-170). New York, NY: Routledge.

Heath, N. L., Schaub, K., Holly, S.., \& Nixon, M. K. (2009). Self-injury today: Review of population and clinical studies in adolescents. In M.K. Nixon and N.L. Heath (Eds.), Self-injury in youth: The essential guide to assessment and intervention (pp. 9-28). New York, NY: Routledge.

Heath, N.L., Toste, J.R., \& Beettam, E.L., (2006). "I am not well-equipped”: High school teachers; perceptions of self-injury. Canadian Journal of School Psychology, 21, 73-92. 
Heath, N. L., Toste, J. R., Sornberger, M. J., \& Wagner, C. (2011). Teachers' perceptions of non-suicidal self-injury in the schools. School Mental Health, 3, 35-43.

Henggeler, S. W., Schoenwald, S. K., Borduin, C. M., Rowland, M. D., \& Cunningham, P. B. (2009). Multisystemic therapy for antisocial behavior in children and adolescents. (2nd ed.). New York: Guilford.

Hilt, L.M., Nock, M.K., Lloyd-Richardson, E.E., \& Prinstein, M.J. (2008). Longitudinal study of nonsuicidal self-injury among young adolescents. Journal of Early Adolescence, 28, 455-469.

Huband, N. \& Tatum, D. (2000). Attitudes to self-injury within a group of mental health staff. British Journal of Medical Psychology, 73, 495-504.

Huey, S. R., Henggeler, S. W., Rowland, M. D., Halliday-Boykins, C. A., Cunningham, P. B., Pickrel, S. G., \& Edwards, J. (2004). Multisystemic therapy effects on attempted suicide by youths presenting psychiatric emergencies. Journal Of The American Academy Of Child \& Adolescent Psychiatry, 43, 183-190.

Individuals with Disabilities Education Improvement Act. (2004). Building the legacy, IDEA 2004. Retrieved January 15, 2010, from http://idea.ed.gov/

Israel, J.A., \& Lee, K. (2002). Amphetamine usage and genital self-mutilation. Addiction, $97,1215-1218$.

Jacobson, C.M., Muehlenkamp, J. J., Miller, A.L., \& Turner, B. (2008). Psychiatric impairment among adolescents engaging in different types of deliberate selfharm. Journal of Clinical Child \& Adolescent Psychology, 37, 363-375.

Kahan, J., \& Pattison, E. (1984). Proposal for a distinctive diagnosis: the deliberate selfharm syndrome (DSH). Suicide \& Life-Threatening Behavior, 14, 17-35. 
Kaplowitz, M. D., Hadlock, T. D., \& Levine, R. (2004). A Comparison of Web and Mail Survey Response Rates. Public Opinion Quarterly, 68, 94-101.

Kaplan, R. M., \& Saccuzzo, D. P. (2001). Psychological Testing: Principles, Applications and Issues ( $5^{\text {th }}$ ed.). Belmont, CA: Wadsworth/Thompson Learning.

Keppel, G, \& Wickens, T.D. (2004). Design and Analysis: A Researcher's Handbook (4 ${ }^{\text {th }}$ ed). Upper Saddle River, NJ: Pearson Prentice Hall.

Kirkcaldy, B.D., Brown, J.M., \& Siefen, G. R. (2007). Profiling adolescent attempting suicide and self-injurious behavior. International Journal on disability and human development, $6,75-86$.

Klonsky, E., Muehlenkamp, J. J., Lewis, S. P., \& Walsh, B. (2011). Nonsuicidal selfinjury. Cambridge, MA US: Hogrefe Publishing.

Klonsky, E.D., \& Olino, T.M. (2008). Identifying clinically distinct subgroups of selfinjurers among young adults: A latent class analysis. Journal of Consulting and Clinical Psychology, 76, 22-27.

Klonsky, E., \& Weinberg, A. (2009). Assessment of nonsuicidal self-injury. In M. K. Nock (Ed.). Understanding nonsuicidal self-injury: Origins, assessment, and treatment (pp. 183-199). Washington, DC US: American Psychological Association.

Kratochwill, T.R., \& Shernoff, E.S. (2004). Evidence-based practice: promoting evidence-based interventions in school psychology. School Psychology Review, $33,34-48$.

Langsrud, O. (2003), ANOVA for unbalanced data: Use Type II instead of Type III Sums of Squares. Statistics and Computing, 13, 163-167. 
Levenkron, S. (1998). Cutting: Understanding and overcoming self-mutilation. New York: W.W. Norton \& Company, Inc.

Lewis, S., Heath, N., St Denis, J., \& Noble, R. (2011). The scope of nonsuicidal selfinjury on YouTube. Pediatrics, 127, 552-557.

Lieberman, R.A., Toste, J. R., \& Heath, N. L. (2009). Nonsuicidal self-injury in the schools: Prevention and intervention. In M.K. Nixon and N.L. Heath (Eds.), Selfinjury in youth: The essential guide to assessment and intervention (pp.195-216). New York, NY: Routledge.

Linehan, M. M. (1993). Cognitive behavioral treatment of borderline personality disorder. New York: Guilford Press.

Lloyd, E., Kelley, M. L., Hope, T. (1997). Self-mutilation in a community sample of adolescents: Descriptive characteristics and provisional prevalence rates. Paper presented at the Annual Meeting of the Society for Behavioral Medicine, New Orleans, LA.

Lloyd-Richardson, E., Nock, M., \& Prinstein, M. (2009). Functions of adolescent nonsuicidal self-injury. In M.K. Nixon and N.L. Heath (Eds.), Self-injury in youth: The essential guide to assessment and intervention (pp.29-41). New York, NY: Routledge.

Lloyd-Richardson, E. E., Perrine, N., Dierker, L., \& Kelley, M.L. (2007). Characteristics and functions of non-suicidal self-injury in a community sample of adolescents. Psychological Medicine, 37, 2007.

Lofthouse, N., Muehlenkamp, J.J., \& Adler, R. (2009). Nonsuicidal self-injury and cooccurrence. In M.K. Nixon and N.L. Heath (Eds.), Self-injury in youth: The 
essential guide to assessment and intervention (pp.59-78). New York, NY:

Routledge.

Matsumoto, T., Yamaguchi, A., Chiba, Y., Asami, T., Iseki, E., \& Hirayasu, Y. (2004).

Patterns of self-cutting: A preliminary study on differences in clinical implications between wrist- and arm,-cutting using a Japanese juvenile detention center. Psychiatry and Clinical Neurosciences, 58, 377-382.

Miller, A. L., Rathus, J. H., \& Linehan, M. M. (2006). Dialectical behavior therapy with suicidal adolescents. New York, NY: Guilford Publications.

Miller, D. N., \& Brock, S. E. (2010). Identifying, assessing, and treating self-injury at school. New York, NY US: Springer Science

Moor, S., Ann, M., McQueen, H., Wells, J. E., Robert, E., \& Robert, W., et al (2007). Improving the recognition of depression in adolescence: Can we teach the teachers? Journal of Adolescence, 30, 81-95.

Moore, C.R. (2009). Examination of self-injury and related interventions knowledge of school-based health and mental health professionals. Unpublished master's manuscript. University of Missouri, Columbia, MO.

MU Partnership for Education Renewal (MPER) (2009). Teaching Fellow Program. Retrieved January 15, 2010, from http://education.missouri.edu/orgs/mper/fellows/

National Institute for Health Care Management Foundation. (2005, February). Children's mental health: An overview and key considerations for health system stakeholders. Washington, DC: Author. 
National Mental Health Association. (2006). Access to mental health care. Retrieved March, 2008, from http://www.nmha.org/.

Newhill, C. E., Eack, S. M., \& Conner, K. O. (2009). Racial differences between African and White Americans in the presentation of borderline personality disorder. Race And Social Problems, 1, 87-96.

Nixon, M. K., Aulakh, H., Townsend, L., \& Atherton, M. (2009). Psychosocial interventions for adolescents. In M.K. Nixon and N.L. Heath (Eds.), Self-injury in youth: The essential guide to assessment and intervention (pp.217-236). New York, NY: Routledge.

Nixon, M., Cloutier, P., \& Jansson, S. (2008). Nonsuicidal self-harm in youth: a population-based survey. CMAJ: Canadian Medical Association Journal, 178, 306-312.

Nixon, M. K., \& Heath, N. L. (2009). Self-injury in youth: The essential guide to assessment and intervention. New York, NY US: Routledge/Taylor \& Francis Group.

Nock, M. K. (2008). Understanding nonsuicidal self-injury: Origins, assessment and treatment. Washington, DC: American Psychological Association.

Nock, M.K., Borges, G., Bromet, E.J., Alonso, J., Angermeyer, M., \& Beautrais, A., et al. (2008). Cross-national prevalence and risk factors for suicidal ideation, plans, and attempts. The British Journal of Psychiatry, 192, 98-105.

Nock, M. K., \& Cha, C. B. (2009). Psychological models of nonsuicidal self-injury. In M. K. Nock (Ed.). Understanding nonsuicidal self-injury: Origins, assessment, and treatment (pp. 65-77). Washington, DC US: American Psychological Association. 
Nock, M.K., Holmberg, E.B., Photos, V.I., \& Michel, B.D. (2007). Self-injurious thoughts and behaviors interview: Development, reliability and validity in the adolescent sample. Psychological Assessment, 19, 309-317.

Nock, M. K., Joiner Jr., T. E., Gordon, K.H., Lloyd-Richardson, E., \& Prinstein, M.J. (2006). Non-suicidal self-injury among adolescents: Diagnostic correlates and relation to suicide attempts. Psychiatry Research, 144, 65-72.

Nock, M.K., \& Mendes, W.B. (2008). Physiological arousal, distress tolerance, and social problem -solving deficits among adolescent self-injurers. Journal of Consulting and Clinical Psychology, 76, 28-38.

Nock, M. K., \& Prinstein, M. J. (2004). A functional approach to the assessment of selfmutilative behaviors. Journal of Consulting and Clinical Psychology, 72, 885890.

Olobatuyi, M. E. (2006). A user's guide to path analysis. Lanham, MD:University Press of America.

Olson, C.L. (1976). On choosing a test statistic in multivariate analysis of variance. Psychological Bulletin, 83, 579-586.

Onwuegbuzie, A. J., \& Leech, N. L. (2004). Post hoc power: A concept whose time has come. Understanding Statistics, 3, 201-230.

Ormrod, J. E. (2004). Human learning (4th ed.). Upper Saddle River, NJ: Merrill/Prentice Hall.

Ormrod, J. E. (2008). Human learning (5th ed.). Upper Saddle River, NJ: Merrill/Prentice Hall. 
Ormrod, J. E. (2009). Essentials of educational psychology (2nd ed.). Upper Saddle River, NJ:

Merrill/Prentice Hall.

Pedhazur, E. J. (1997). Multiple regression in behavioral research: Explanation and prediction ( $3^{\text {rd }}$ ed.). Belmont, CA: Wadsworth-Thomson Learning.

Preacher, K. J., \& Hayes, A. F. (2008). Asymptotic and resampling strategies for assessing and comparing indirect effects in multiple mediator models. Behavior Research Methods, 40, 879-891.

Prinstein, M.J., Nock, M.K., Simon, V., Aikins, J.W., Cheah, C.S.L., \& Spirito, A. (2008). Longitudinal trajectories and predictors of adolescent suicidal ideation and attempts following inpatient hospitalization. Journal of Consulting and Clinical Psychology, 76, 92-103.

Reinke, W. M., Stormont, M., Herman, K. C., Puri, R., \& Goel, N. (2011). Supporting children's mental health in schools: Teacher perceptions of needs, roles, and barriers. School Psychology Quarterly, 26, 1-13.

Richardson, J. E. (2011). Eta squared and partial eta squared as measures of effect size in educational research. Educational Research Review, 6, 135-147.

Rollinik, J. D., Schneider, U., Wedegaertner, F., Huber, T. J., \& Emrich, H. M. (2001). Uncommon self-mutilation in a borderline personality disorder patient. Bipolar Disorders, 3, 133-134.

Ross, S., \& Heath, N. (2002). A study of the frequency of self-mutilation in a community sample of adolescents. Journal of Youth and Adolescence, 31, 67-77. 
Schianlge, M. (2002). Recurrent suicide attempts, self-mutilation, and binge/purge behavior: A case report. Harvard Review Psychiatry, 10, 353-363.

Shapiro, S. (2008). Addressing self-injury in the school setting. The Journal of School Nursing, 24, 124-130.

Share Shame. (n.d.). Self-Injury. Retrieved October 8, 2005, from http://www.palace.net/ llama/psych/pharm.html

Sobel, Michael E. (1982). Asymptotic confidence intervals for indirect effects in structural equation models. Sociological Methodology, 13, 290-312.

Stevens, J.P. (2001). Applied multivariate statistics for the social sciences. Mahwah, NJ: Lawrence Erlbaum Associates

Stirn, A., \& Hinz, A. (2008). Tattoos, body piercings, and self-injury: Is there a connection? Investigations on a core group of participants practicing body modification. Psychotherapy Research, 18, 326-333.

Tabachnick, B.G., \& Fidell, L.S. (2007). Using Multivariate Statistics (5 ${ }^{\text {th }}$ ed.). Boston, MA: Allyn and Bacon

Tabachnick, B.G., \& Fidell, L.S. (2012). Using Multivariate Statistics ( $6^{\text {th }}$ ed.). Boston, MA: Allyn and Bacon

Wallenstein, M.B., \& Nock, M.K. (2007). Physical exercise as a treatment for nonsuicidal self-injury: Evidence from a single-case study. American Journal of Psychiatry, 164, 350-351.

Walsh, B. (2005) Treating self-injury: A practical guide. New York, NY: Guilford Press. Waska, R. T. (1998). Self-mutilation, substance abuse, and the psychoanalytic approach: Four cases. American Journal of Psychotherapy, 52, 18-27. 
Weinhurt, K.P. (1995) Multivariate analysis of variance. In L.G. Grimm and P.R. Yarnold (Eds.) Reading and understanding multivariate statistics. Washington DC: American Psychological Association.

Wenar, C. \& Kerig, P. (2006). Developmental psychopathology: From infancy through adolescence. New York: McGraw-Hill.

Whittaker, T. (2005, September). Lecture on testing assumptions in Multiple Analysis of Variance (MANOVA). Notes presented at University of Missouri-Columbia. Columbia, MO.

Woolfolk, A. (2010). Educational psychology (1 $1^{\text {th }}$ ed.). Boston, MA: Merrill

White, J., Leggett, J., \& Beech, A. (1999). The incidence of self-harming behaviour in the male population of a medium-secure psychiatric hospital. The Journal of Forensic Psychiatry, 10, 59-68.

Yates, T. M., Tracy, A. J., \& Luthar, S. S. (2008). Nonsuicidal self-injury among "privileged" youths: Longitudinal and cross-sectional approaches to developmental process. Journal of Consulting and Clinical Psychology, 76, 5262.

Youngs, P., Jones, N., \& Low, M. (2011). How beginning special and general education elementary teachers negotiate role expectations and access professional resources. Teachers College Record, 113, 1506-1540.

Zila, L.M., \& Kiselica, M.S. (2001). Understanding and counseling self-mutilation in female adolescents and young adults. Journal of Counseling \& Development, 79, 46-52. 
Zoroglu, S.S., Tuzun, U., Sar, V., Tutkun, H., Savas, H. A., Ozturk, M., Alyanak, B., \& Kora, M. E. (2002). Suicide attempt and self mutilation among Turkish high school students in relation with abuse, neglect and dissociation. Psychiatry and Clinical Neurosciences, 57, 119-126. 
APPENDIX 


\section{Appendix 1}

University of Missouri-Columbia

College of Education

16 Hill Hall

Department of Educational, School, Counseling Psychology

Columbia, MO 65211

Phone: (573) 814-9537

Craig Frisby, Ph.D.

Caren Moore

CarenMoore@mail.mizzou.edu
Email:CLFrisby@missouri.edu

Email:

Dear Prospective Participant:

This consent form is designed to ask your permission for participating in the current survey regarding non-suicidal self-injurious behaviors of youngsters.

Research Purpose: This letter politely requests your participation in the study of NonSuicidal Self-Injury Survey for School-Based Professionals. The primary purpose of this study is to gather information regarding school-based professional's knowledge base of non-suicidal self-injurious behaviors. Our objective is to better understand the general knowledge, recommendations, and self-efficacy in providing those recommendations of school-based professionals with regarding to non-suicidal self-injurious behaviors so that implications and recommendations may be made regarding professional development and training.

Procedure: To achieve the study's purpose, your responses to an online questionnaire will be used. The questionnaire consists of questions regarding experience with selfinjurious students, conceptualization of self-injury, identification of intervention and prevention measures, and self-efficacy of providing those interventions. It will take approximately 8 to 10 minutes to complete the questionnaire.

Voluntary Participation: Your participation is completely voluntary, and you may withdraw from the study at any time.

Anonymity: All records and information collected in this study are completely anonymous. The data will be stored for seven years following the completion of the study in the researcher's personal computer by federal regulations and will be only accessible by the researcher. Name and any data that could identify the participants will not be obtained. All email addresses that will be collected for the raffle will be destroyed after incentives have been distributed. In any reporting of the data all individuals will be anonymous and aggregated, so there is no risk of your individual participation in this study becoming known. 
Result Sharing: Results of this research will be presented in a dissertation, possible future publications as well as poster presentations at state and national conferences. Findings will be presented in aggregate form with no personal identifiers.

Benefits: The findings from this research can be used to improve school-based professionals' understanding of and ability to intervene with self-injurious behaviors. All districts will be provided with an executive summary of the finding, by professional role, for their district with for professional development opportunities.

Risks: We do not foresee any risks or discomforts beyond those you normally experience as a result of your participation in a research study.

Questions: If you have further questions, you may reach Caren Moore at CarenMoore@mail.mizzou.edu. For additional information regarding human participation in research, please contact the University of Missouri Campus Institutional Board at (573) 882-9585.

To give consent you must be 18 years of age or older. By continuing with the survey, you are identifying yourself as 18 years of age and older as well as consenting to participate in this research study. If you are interested in the final results of this study, please email Caren Moore at CarenMoore@mail.missouri.edu with your contact information.

Thank You,

Caren Moore, M. A.

Doctoral Candidate and Graduate Instructor

Craig Frisby, Ph.D.

Associate Professor 


\section{Appendix 2}

\section{Examination of School-Based Professionals' General Knowledge of Self-Injury and Related Interventions}

Study Overview: It is estimated that $5-20 \%$ of the school age population engages in selfinjurious behaviors (e.g., cutting, burning, bruising, etc.). The researcher would like to examine school-based personnel's general knowledge, knowledge of interventions and capacity to provide interventions with respect to non-suicidal self-injurious behaviors.

Study Participants Seeking: Teachers, school counselors, school psychologists, school social workers, paraprofessionals, administrators, school psychological examiners, and school nurses

Estimated Survey Time: 8-10 minutes

Administration of Survey: The survey shall be administered via a link to SurveyMonkey. Upon approval, each school or district administrator will disseminate the survey through their respective listservs. A short paragraph about the survey will be sent to the district administrator along with the link for administration.

http://www.surveymonkey.com/s/nonsuicidalselfinjurysurvey

Benefits for School District: Each participating district will receive an executive summary reporting results by professional position or aggregated, depending on preference and available data. This information may be used as an indicator for potential professional development needs for the district.

Benefits for Individual Persons: Participants will have the opportunity to enter a raffle for 1 of $20 \$ 20$ Mastercard/Visa gift cards by providing their email address at the end of the survey. Please click or copy and paste the link in your browser to enter the raffle http://www.surveymonkey.com/s/nonsuicidalselfinjuryraffle

Protection of Participant's Information: No home or school addresses, phone numbers or other identifying information will be collected. All survey responses are anonymous. Aggregate data by role will be provided to the district for professional development purposes only. Email addresses will be collected for the raffle. Afterwards, all email addresses will be securely discarded once incentives have been disbursed.

Window of participation: The survey is currently ready for dissemination. The survey will be open until the end of March 2013. A reminder email will be sent two weeks after the initial administration of the survey. A final message will be sent advising potential participants of the survey close date.

Contact Information for Researcher:

Caren R. Moore

University of Missouri 
16 Hill Hall

Columbia, MO 65211

Phone: 573-489-3569

carenmoore@mizzou.edu 


\section{Appendix 3}

Hello,

- Non-suicidal self-injury-It is estimated that $5-20 \%$ of the school age population engages in self-injurious behaviors (e.g., cutting, burning, bruising, etc.). I would like to examine school-based professional's general knowledge, knowledge of interventions, and self-efficacy with respect to non-suicidal self-injurious behaviors.

- How do I participate? The survey shall be administered via a link to SurveyMonkey. Click or copy and paste the following link into your browser to access the survey. http://www.surveymonkey.com/s/nonsuicidalselfinjurysurvey

- Incentives? Participants will have the option to enter a raffle for 1 of $10 \$ 25$ Mastercard gift cards by providing their email address at the end of the survey. Please click or copy and paste the link in your browser to enter the raffle upon completion of the survey.

http://www.surveymonkey.com/s/nonsuicidalselfinjuryraffle

- Anonymous and Confidential-No home or school addresses, phone numbers or other identifying information will be collected. All survey responses are anonymous. Data will be summarized and presented to districts for professional development purposes. Email addresses will be collected for the raffle. Afterwards, all email addresses will be securely discarded once incentives have been disbursed.

Thank you for your participation!

Caren Moore, M.A.

School Psychology Doctoral Candidate 


\section{Appendix 4}

\section{Script for School-Based Professionals Regarding Non-Suicidal Self-injury Study}

Hello. How are you? (Reply to response: Great, Wonderful, or Terrific) My name is Caren

Moore and I am a graduate student in the school psychology program at the University of Missouri-Columbia. I am calling today to ask for your district/organization and personal participation in a study regarding non-suicidal self-injury.

Non-Suicidal Self-Injury is an individual's intentional harm or insult to the body without the intent to commit suicide. This does not include harming oneself due to a developmental disability such as autism or mental retardation. It is estimated that 5-8\% of the school age population engages in self-injurious behaviors (e.g., cutting, burning, bruising, etc.). The researcher would like to examine school-based personnel's general knowledge, knowledge of interventions and capacity to provide interventions with respect to non-suicidal self-injurious behaviors. We are seeking teachers, school counselors, school psychologists, school social workers, paraprofessionals, administrators, school psychological examiners, and school nurses; essentially all schoolbased professionals to participate in the study.

The estimated time to complete the survey is 8-10 minutes. The survey will be administered via a link to SurveyMonkey. Upon approval, each school or district administrator will disseminate the survey through their respective listserv. No email addresses, home or school addresses, phone numbers or other identifying information will be collected. All survey responses are anonymous. Aggregate data by role shall be provided to the district solely for professional development purposes.

The survey will be ready for dissemination beginning March 1, 2011. The survey will be open for until the end of April. A reminder email will be sent two weeks after the initial administration of the survey. One week later, a final message will be sent advising potential participants of the close date of the survey.

Here are some incentives for your district and professionals working in your district: Each participating district will receive an executive summary broken down by professional position or aggregated indicating professional development needs for the district. Also, participants will have the option of entering into a raffle for 1 of $10 \$ 25$ Mastercard gift cards.

\section{Would you like more information regarding the study?}

If yes: Thank you for your time and interest. I can give you the name of the student investigator, Caren Moore. May I have your contact information so that the student investigator can provide you with more information. 
Would you like her contact information? Her contact information is 573-8149537 or CarenMoore@mizzou.edu She would be more than happy to speak with you further regarding the study.

Again, thank you for your time. Have a good day!

If no: Thank you for your interest. I am ecstatic that you would like to participate in the study. May I have your contact information to send you the link for distribution? The link has the consent form and information about the study. Again, thank you for your time and participation. If you have any questions or concerns please feel free to contact the student investigator, Caren Moore, at is 573-814-9537 or CarenMoore@mizzou.edu She would be more than happy to speak with you further regarding the study.

Have a good day! 
Appendix 5

\section{THANK YOU FOR YOUR TIME!}

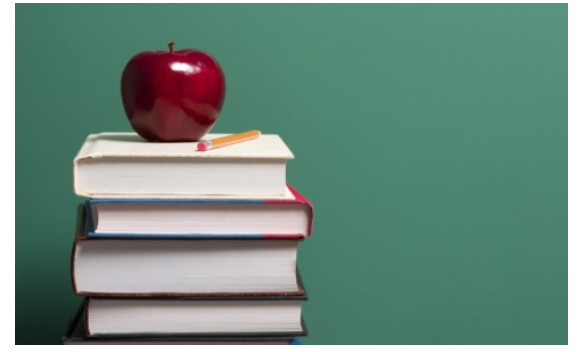

If you would like more information regarding the non-suicidal self-injury study, please provide your contact

information below:

Name

School District

Phone Number

Email Address

\section{THANK YOU FOR YOUR TIME!}

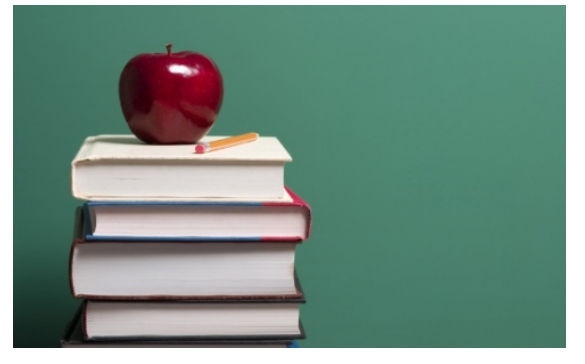

If you would like more information regarding the non-suicidal self-injury study, please provide your contact

information below:

Name

School District 
Phone Number

Email Address 
Appendix 6

Assessment of School-Based Staff Knowledge and Self-Efficacy Related to NSSI

Demographic Section

1. Please identify your school district. District

2. What area of the country is your district located?

1. North

2. South

3. West

4. East

5. Midwest

3. What role do you serve within your school? If you serve multiple roles, indicate your primary role (60\% or more of your time).

1. School Counselor

2. School Psychologist

3. School Psychological Examiner

4. Regular Education Teacher

5. Special Education Teacher

6. School Social Worker

7. School Nurse

8. School/District Administrator (i.e., principal)

9. Other, please specify

4. Please indicate the highest degree attained.

1. High School Diploma

2. Associates

3. Bachelors

4. Masters

5. Education Specialist (Ed.S.)

6. Doctorate (Ph.D., Ed.D., etc.)

5. What type of school are your responsible for? If you are assigned to multiple schools, indicate the school for which you are primarily responsible $(60 \%$ or more of your time).

1. Preschool/Early Childhood

2. Elementary

3. Middle School

4. Junior High School

5. High School

6. Other, Please Specify 
6. Do you have direct experience in working with individuals who engage in nonsuicidal self-injurious behaviors (e.g., cutting, harming oneself without suicidal intent)?

1. None

2. One or two experiences

3. Some experience

4. Regular experiences

5. Very Regular experiences

7. Please indicate the experience you have had with children related to the following mental health issues (Check all that apply):

1. Depression (Prolonged Sad Mood)

2. Anxiety

3. Obsessive Compulsive Disorder

4. Oppositional Defiant Disorder

5. Conduct Disorder

6. Tourette's Syndrome

7. Non-Suicidal Self-Injury

8. Eating Disorders 


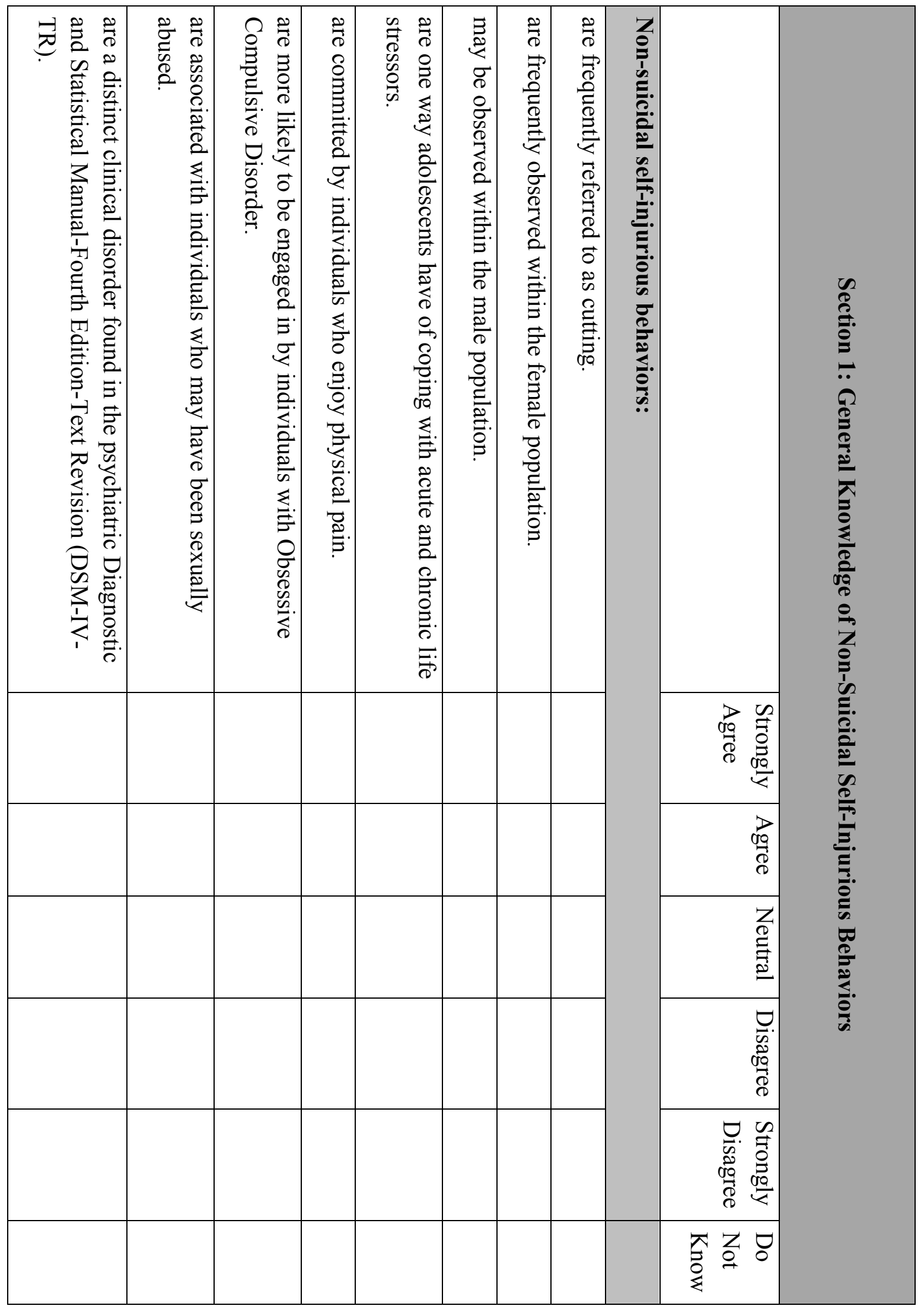




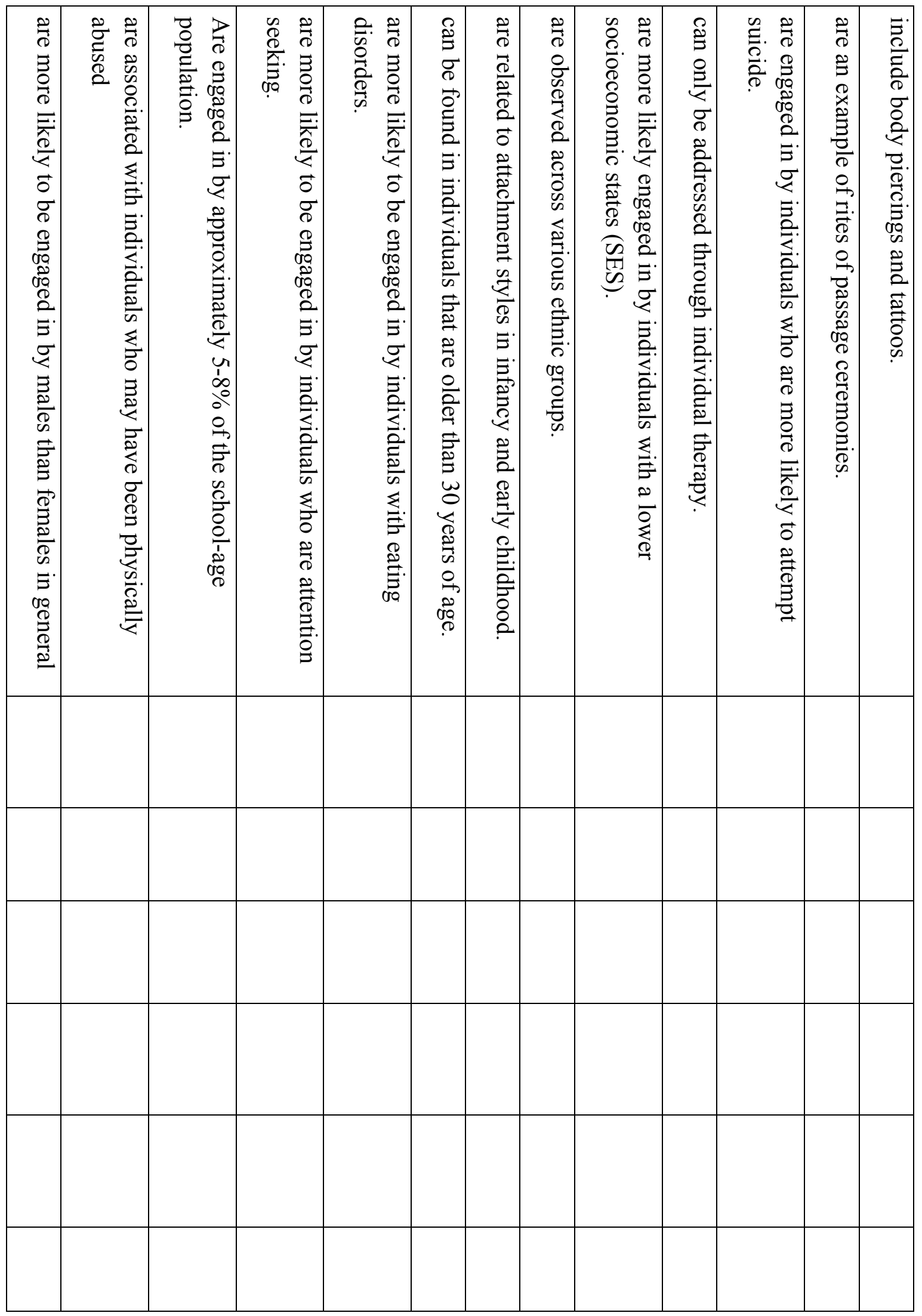




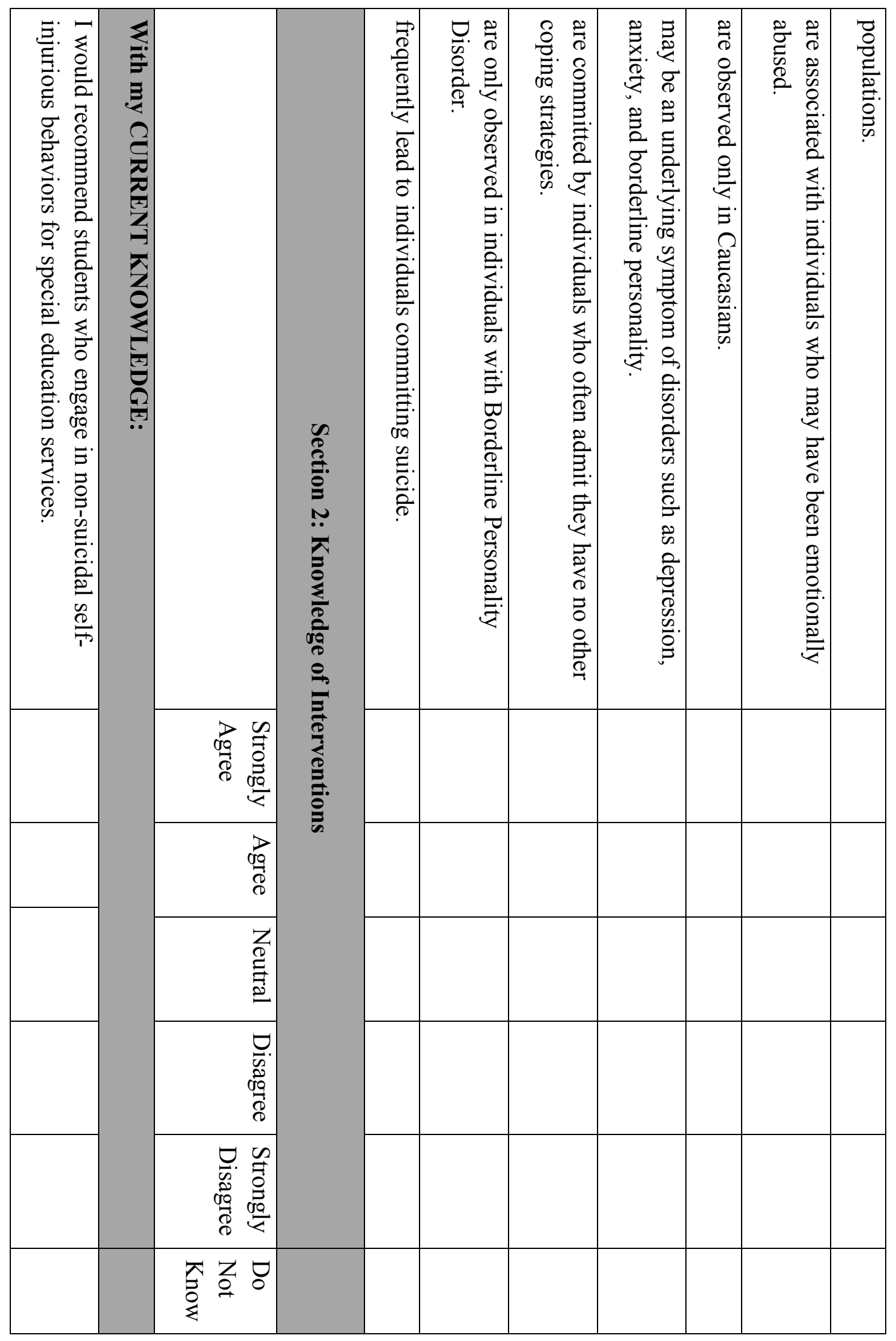




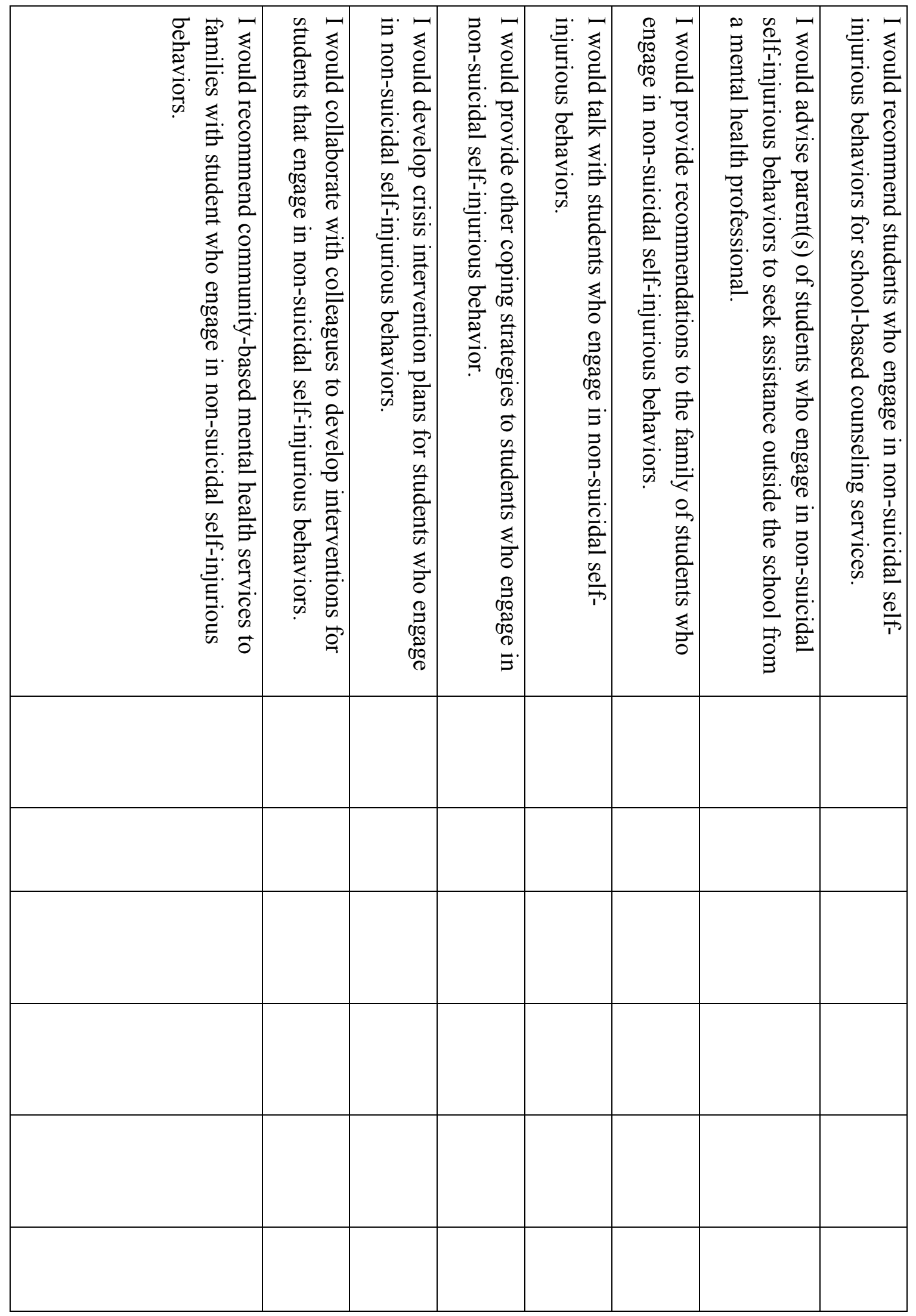




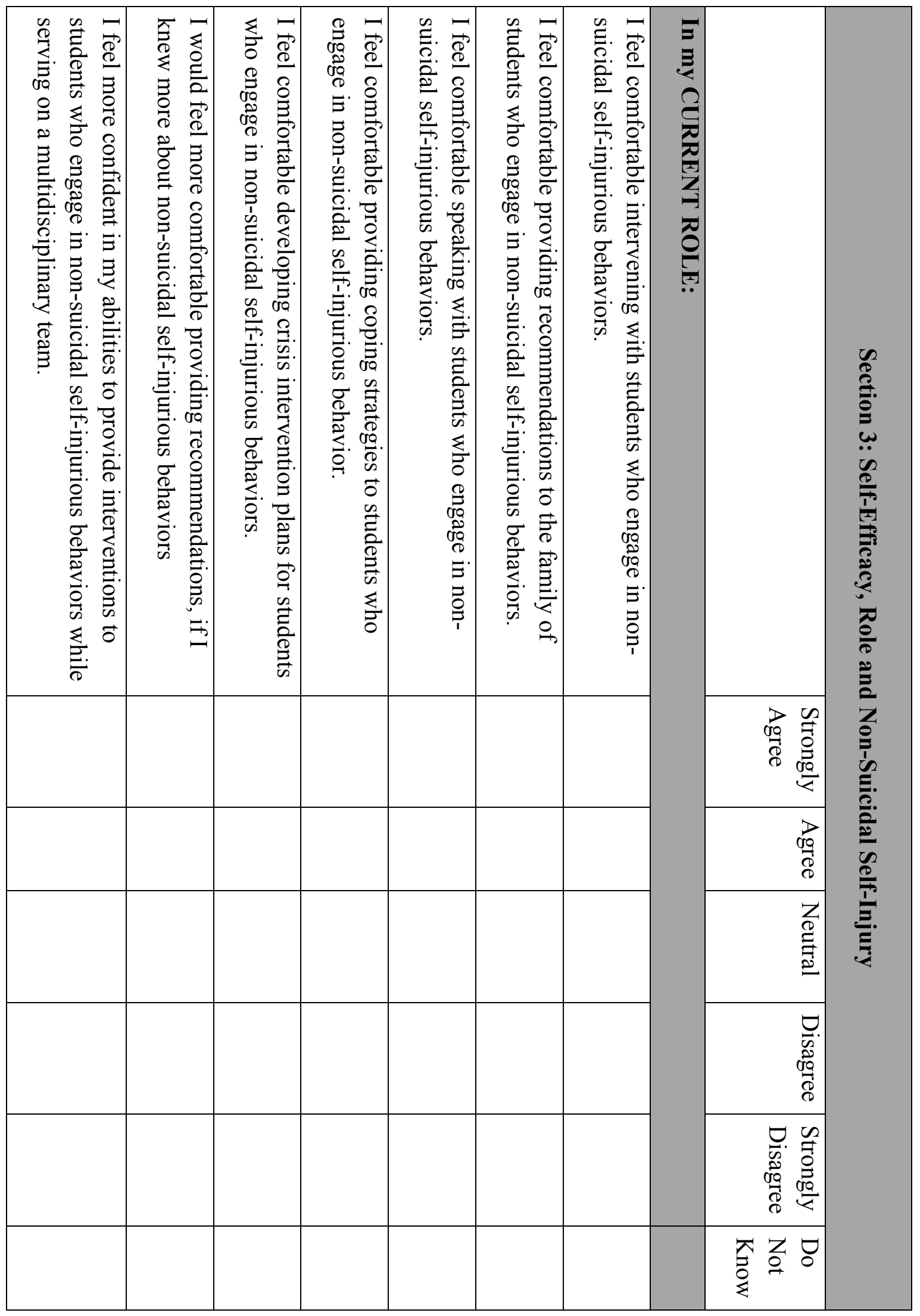



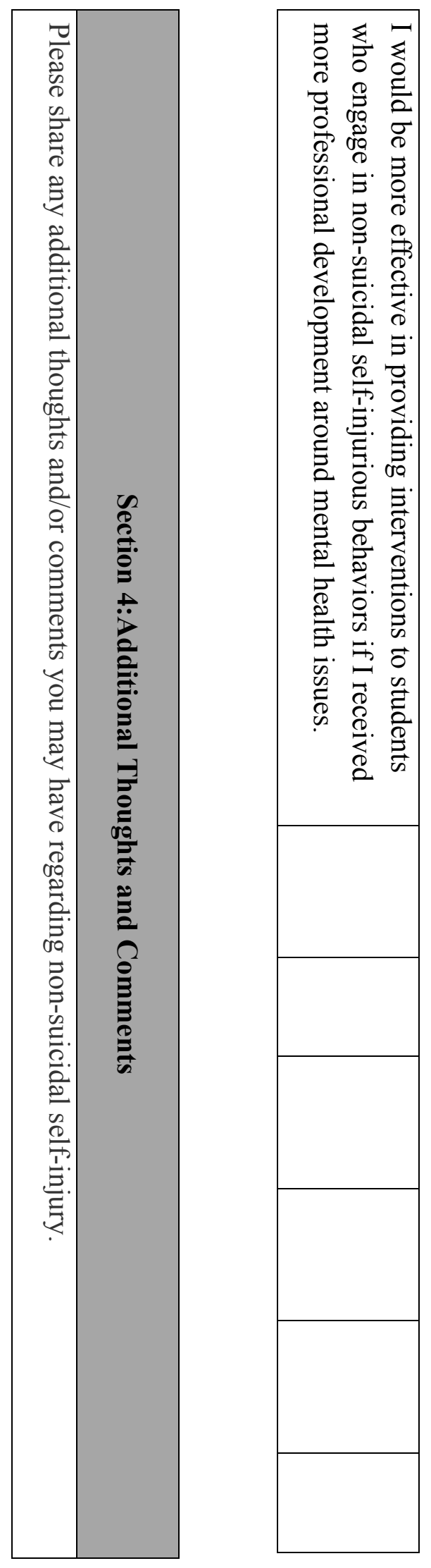


\section{VITA}

I am a native of Little Rock Arkansas. I began my collegiate career at the University of Arkansas-Fayetteville and finished up at the University of Arkansas at Little Rock majoring in Psychology and minoring in Sociology. After graduating, I moved to Columbia, Missouri to complete my doctorate in Educational and Counseling Psychology with an emphasis in School Psychology. With the culminating experience of an internship at the Boone County Juvenile Office, Columbia College and Columbia Public Schools, I conferred my Ph.D. May 2015.

My graduate degrees have a heavy emphasis on research and related clinical practice with children, adolescents, adults, and families. I have skills in implementing strength-based and person-centered interventions to improve the overall functioning of children, adolescents, and families. I have spent the last 11 years providing direct and indirect services to children, adolescents, adults, and families. I have two beautiful daughters and will be relocating to the Little Rock area for employment. 